\title{
Anisotropic Nanomaterials: Silver-Copper Oxide Core-Partial Shell Nanoparticles
}

by

\section{Emma Jorgenson}

A thesis submitted to the Faculty of Graduate and Postdoctoral Affairs

In partial fulfillment of the requirements for the degree of

Master of Science

in

Chemistry

Carleton University

Department of Chemistry

Ottawa, Ontario, Canada

April 2017

(C) 2017, Emma Jorgenson 
The undersigned hereby recommends to the

Department of Chemistry

acceptance of the thesis

\title{
Anisotropic Nanomaterials: Silver-Copper Oxide Core-Partial Shell Nanoparticles
}

\author{
Submitted by: \\ Emma Jorgenson \\ In partial fulfillment of the requirements of the degree of \\ Master of Science in Chemistry
}

Dr. Anatoli Ianoul

(Supervisor) 


\section{Abstract}

Anisotropic materials have a variety of unique properties and potential applications, including catalysis and directed assembly. As such, production of anisotropic nanoparticles is of interest. In this work, the production of core-partial shell silver nanocube-copper oxide nanoparticles, is the goal. To produce these particles, the silver nanocubes are deposited onto polystyrene thin films, and are embedded into the polymer to a controlled depth. This allows for control over the exposed surface, which is the surface available for coating with copper oxide. These nanoparticles can be removed from the surface by dissolving the polystyrene, allowing their in-solution properties to be probed.

In order to produce these structures, several set-ups were tried, leading to the development of a promising final set-up. Each of the set-ups had different stirring rates, stability of the attached slide and production of bubbles, which can interfere with the production of copper oxide shells.

With the initial set-ups, the order and rate of addition of the reducing agent, hydrazine, or the copper precursor, copper nitrate, were varied, with and without lactic acid and for both supported and embedded nanocubes. The produced morphologies varied for different order and rate of addition of precursors, therefore, allowing for control over the produced morphology by changing these parameters.

With the final set-up, an investigation on the amount of copper precursor, sodium hydroxide, and lactic acid for a constant amount of reducing agent was investigated. It was observed that without lactic acid, the produced shells did not increase in coverage 
with increased concentrations. However, with lactic acid, the produced structures increase in thickness from low to intermediate concentrations, but at the highest concentration, very little copper oxide was deposited on the cubes' surfaces, as growth had occurred in solution.

With this type of method, core-partial shell particles can be successfully produced. Future work should involve further effort for the optimization of the order of addition, and concentrations of precursors, for fine control over the produced structures. 


\section{Acknowledgments}

I would like to thank Dr. Anatoli Ianoul for his assistance and support throughout this work. In addition, I am grateful for Dr. Jason Coyle, for all of his help and his neverending supply of ideas. I would also like to acknowledge Dan Prezgot, for his assistance and FDTD simulations. Furthermore, I would like to acknowledge Alexandra GaleMouldey and Joel Halle for their support and for our tea time traditions. Finally, I would like to extend a huge thank you to Danielle McRae and Mike Bushell for their continued support (and proofreading). 


\section{Table of Contents}

1 ABSTRACT

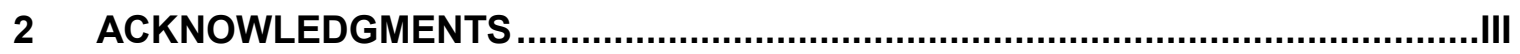

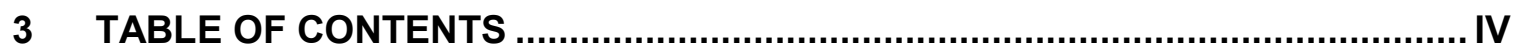

4 TABLE OF FIGURES ……..........................................................................

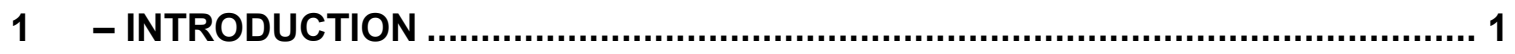

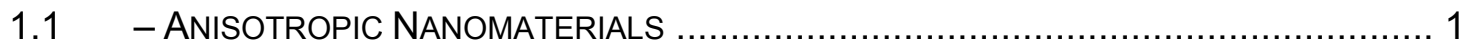

1.1.1 - Applications and Properties of Anisotropic Materials ............................. 2

1.1.2 - Methods of Preparation for Anisotropic Materials .................................. 5

1.1.3 - Overview of the Current Approach to Synthesis of Anisotropic

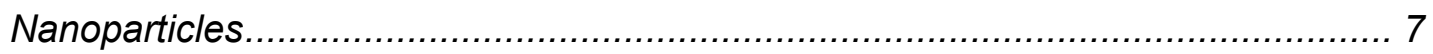

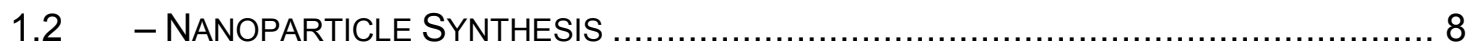

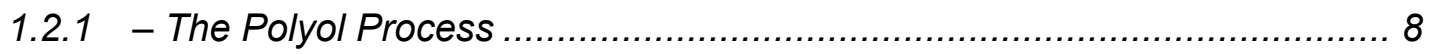

1.2.2 - Nanoparticle Nucleation and Growth ................................................. 9

1.2.3 - Control of Product Morphology: Thermodynamic vs Kinetic Products......12

1.2.4 - Thermodynamic Control Over Product Morphology …............................13

1.2.5 - Kinetic Control Over Product Morphology ..............................................14

1.3 - ANISOTROPIC COPPER OXIDE SHELLS ON SILVER NANOCUBES ......................15

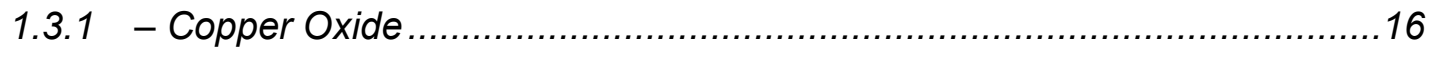

1.3.2 - Copper Oxide Growth...................................................................18

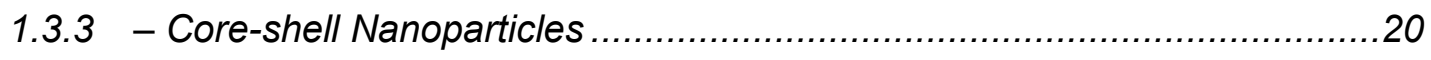

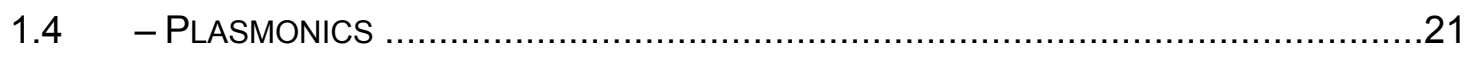

1.4.1 - Surface Plasmon Resonance and Localized Surface Plasmon Resonance 


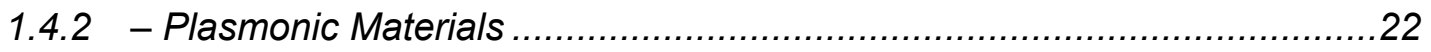

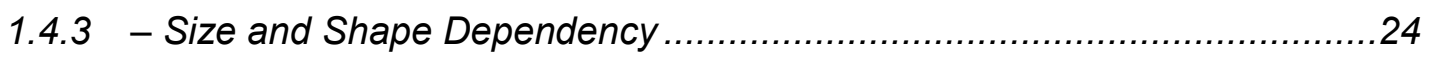

1.4.4 - Environmental Effect on Localized Surface Plasmon Resonances..........25

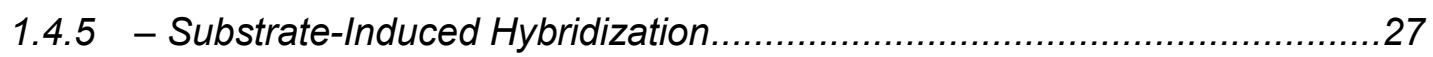

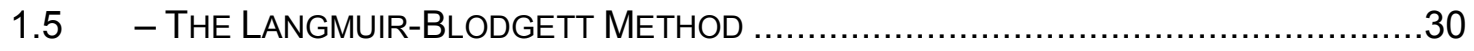

1.5 .1 - Langmuir Monolayer Formation .........................................................

1.5.2 - Measurement of Surface Pressure ......................................................

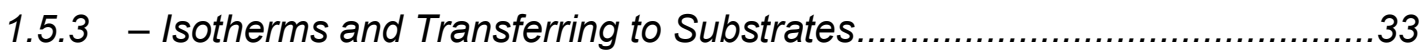

1.6 - POlYMER THIN FILMS AS SUBSTRATES FOR SILVER NANOCUBES.......................34

1.6.1 - Properties of Polymer Thin Films: Glass Transition Temperature .............35

1.6.2 - Nanoparticle Embedding into Polymer Thin Films ................................37

1.6.3 - Spectroscopic Measurement of Silver Nanocube Embedding .................39

1.7 - EXPERIMENTAL SET-UPS FOR COPPER OXIDE GROWTH ................................40

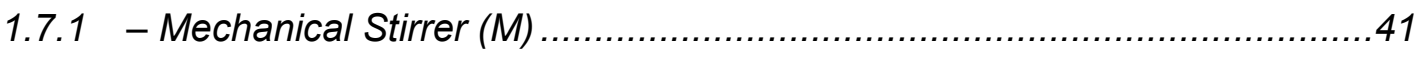

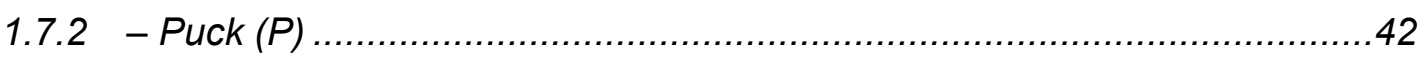

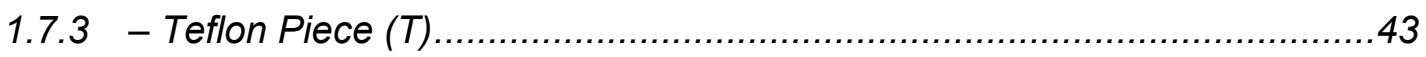

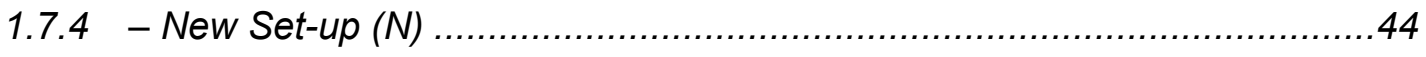

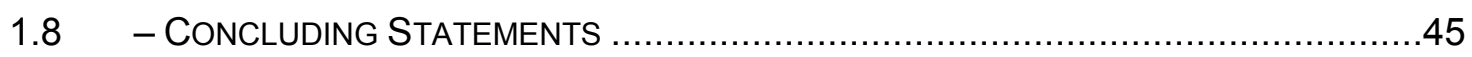

2 - METHODS: COPPER OXIDE COATING ON SILVER NANOCUBES.................47

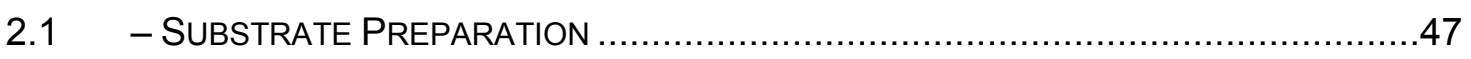

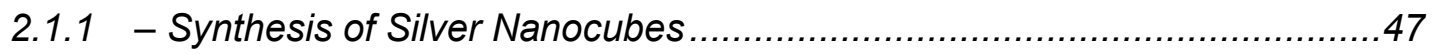

2.1.2 - Preparation of Polystyrene Thin Films ...............................................48

2.1.3 - Langmuir Blodgett Deposition of Silver Nanocubes ..............................48

2.1.4 - Embedding Silver Nanocubes into Polystyrene Thin Films .....................49

2.2 - SET-UPS FOR GROWTH OF COPPER OXIDE SHELLS ...................................49 
2.2.1 - Mechanical Stirrer: Set-up Parameters...............................................

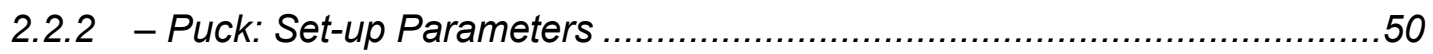

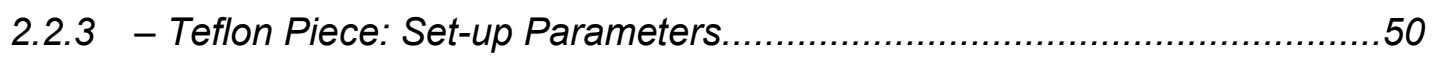

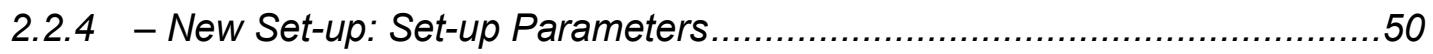

2.3 - SYNTHESIS METHODS OF COPPER OXIDE SHELLS …......................................51

2.3.1 - Copper Oxide Growth: 'Slow' Addition of Hydrazine ...............................51

2.3.2 - Copper Oxide Growth: 'Slow' Addition of Copper Nitrate .........................51

2.3.3 - Copper Oxide Growth: $\mathrm{NaOH}$, Lactic Acid and $\mathrm{Cu}\left(\mathrm{NO}_{3}\right)_{2}$ Concentrations51

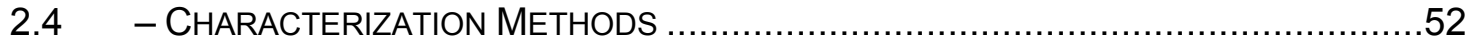

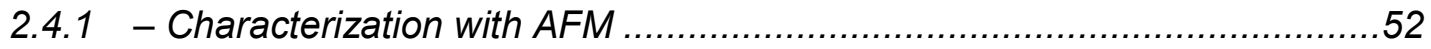

2.4.2 - Removal and Characterization with UV-Vis Spectroscopy and TEM .......52

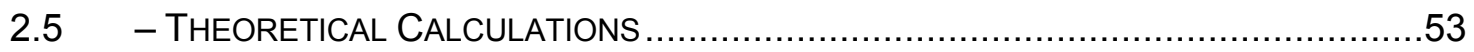

2.5.1 - Finite Difference Time Domain Calculations ........................................53

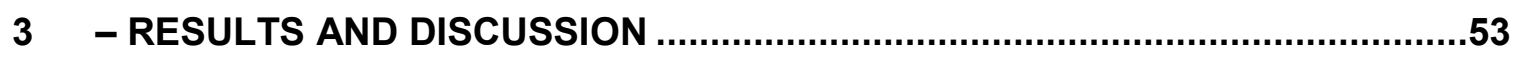

3.1 - PREPARATION OF SUBSTRATES FOR CoPPER OXIDE GROWTH ........................53

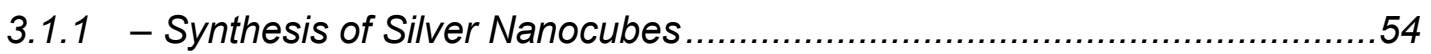

3.1.2 - Preparation of Polystyrene Thin Films ...............................................54

3.1.3 - Langmuir-Blodgett Deposition of Silver Nanocubes...............................55

3.1.4 - Embedding Silver Nanocubes into Polystyrene Thin Films .....................56

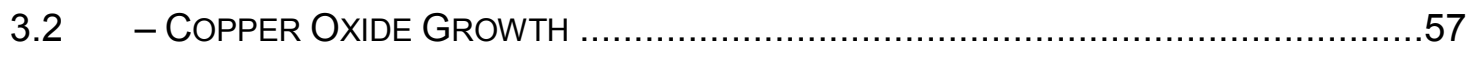

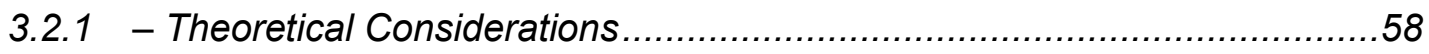

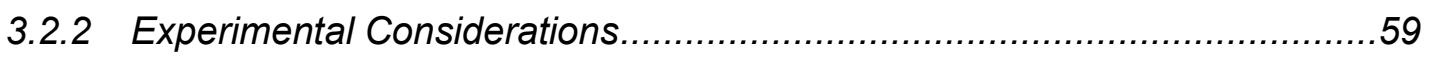

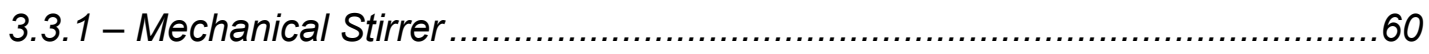

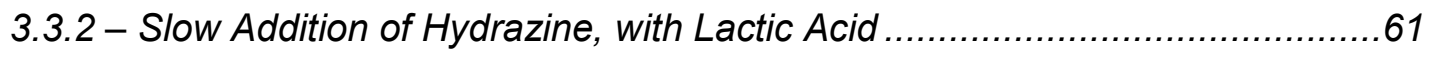

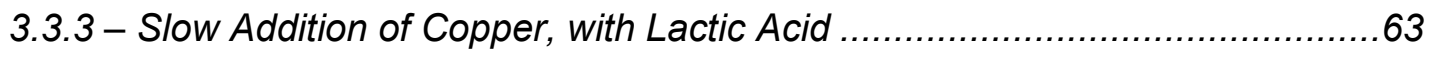


3.3.4 - Slow Addition of Hydrazine, without Lactic Acid ...................................65

3.3.5 - Slow Addition of Copper, without Lactic Acid .....................................66

3.3.6 - Concluding Statements on the Mechanical Stirrer................................68

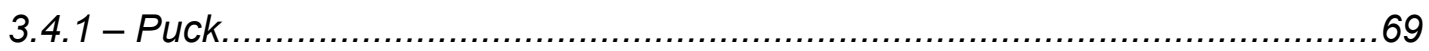

3.4.2 - Slow Addition of Hydrazine, with Lactic Acid .................................... 70

3.4.3 - Slow Addition of Copper, with Lactic Acid ........................................ 71

3.4.4 - Slow Addition of Hydrazine, without Lactic Acid ................................... 73

3.4.5 - Slow Addition of Copper, without Lactic Acid ...................................... 75

3.4.6 - Concluding Statements on the Puck ........................................... 76

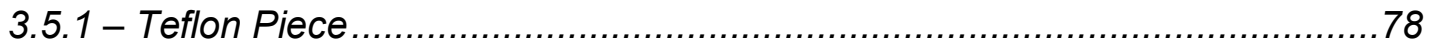

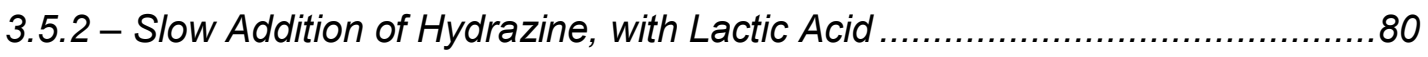

3.5.3 - Slow Addition of Copper, with Lactic Acid ............................................ 82

3.5.4 - Slow Addition of Hydrazine, without Lactic Acid .................................. 85

3.5.5 - Slow Addition of Copper, without Lactic Acid ..................................... 86

3.5.6 - Concluding Statements on the Teflon Piece..................................... 88

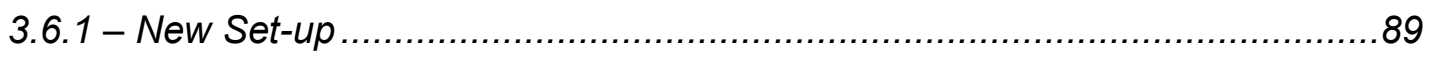

3.6.2 - Hydrazine and Copper Added Quickly, without Lactic Acid ......................90

3.6.3 - Hydrazine and Copper Added Quickly, with Lactic Acid .........................92

3.6.4 - Concluding Statements on the New Set-up.....................................96

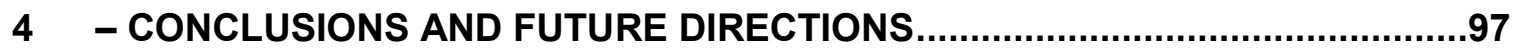

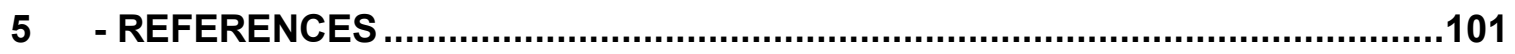




\section{Table of Figures}

Figure 1.1: Schematic of structures with morphological anisotropy, chemical anisotropy and both morphological and chemical anisotropy, where a different colour represents a different material.

Figure 1.2: Schematic of the method of production for core-partial shell silvercopper oxide nanoparticles 7

Figure 1.3: Nucleation of nanoparticles: First reduction generates zero-valent atoms, the concentration rises until it reaches the critical concentration, which allows for nucleation of nanoparticles, which can subsequently grow at concentrations lower than the critical concentration. Reprinted with permission from $\left({ }^{65}\right)$. Copyright $\odot 2012$, Royal Chemical Society.

Figure 1.4: Multiply twinned, singly twinned and single crystalline seeds and produced structures. Reprinted with permission from $\left({ }^{39}\right)$. Copyright $\odot ~ 2011$, American Chemical Society

Figure 1.5: Total free energy of products vs parameters representing structures, indicating for different reaction conditions formation of kinetic products (local minimum) and the thermodynamic product (global minimum). Reprinted with permission from $\left({ }^{66}\right)$. Copyright $@ 2015$, American Chemical Society.

Figure 1.6: $\mathrm{Cu}_{2} \mathrm{O}$ crystal structure and structures of (a) (100), (b) (110) and (c) (111) crystal facets. Reprinted with permission from $\left({ }^{74}\right)$. Copyright $\odot ~ 2015$, Elsevier 
Figure 1.7: Schematic of (a) surface plasmon in a bulk metal and (b) localized surface plasmon resonance (LSPR) in a metal sphere. Reprinted with permission from $\left({ }^{37}\right)$. Copyright $@ 2007$, Annual Reviews.

Figure 1.8: Charge distribution of the $D^{0}$ and $Q^{0}$ modes and the charge distribution of the $D$ and $Q$ modes due to substrate induced hybridization. Adapted with permission from $\left({ }^{97}\right)$. Copyright $@ 2011$, American Chemical Society.

Figure 1.9: UV-Vis spectra of supported silver nanocubes showing sensitivity of $\mathrm{D}$ and $\mathrm{Q}$ hybrid modes to different refractive indices of substrate (left) and surroundings (right). Reprinted with permission from $\left({ }^{107}\right)$. Copyright $@ 2012$, Springer. 30

Figure 1.10: Blodgett deposition (left) and Schaefer deposition (right). Reprinted with permission from $\left({ }^{111}\right)$. Copyright $\odot 2006$, Elsevier. 34

Figure 1.11: Specific volume vs temperature, indicating the glass transition temperature $\left(T_{g}\right)$, glassy and rubbery state for amorphous polymers and the crystalline state and freezing temperature $\left(T_{f}\right)$ for crystalline polymers. 35

Figure 1.12: Plasmonic modes monitored with UV-Vis spectroscopy as silver nanocube monolayers are embedded into polystyrene thin films. Reprinted with permission from $\left({ }^{117}\right)$. Copyright $\odot 2016$, Royal Society of Chemistry. 40

Figure 1.13: Schematic of 'Mechanical Stirrer'. Diagram created with ACD/ChemSketch, version 14.01 Advanced Chemistry Development, Inc., Toronto, On, Canada, www.acdlabs.com, 2015 
Figure 1.14: Schematic of 'Puck'. Diagram created with ACD/ChemSketch, version 14.01 Advanced Chemistry Development, Inc., Toronto, On, Canada, www.acdlabs.com, 2015.

Figure 1.15: Schematic of 'Teflon Piece'. Diagram created with ACD/ChemSketch, version 14.01 Advanced Chemistry Development, Inc., Toronto, On, Canada, www.acdlabs.com, 2015 44

Figure 1.16: Schematic of the 'New Set-up'. Diagram created with ACD/ChemSketch, version 14.01 Advanced Chemistry Development, Inc., Toronto, On, Canada, www.acdlabs.com, 2015. 45

Figure 3.1: (A) UV-Vis spectra of silver nanocubes suspended in ethanol and (B) Atomic force micrograph of silver nanocubes. 54

Figure 3.2: Atomic force micrographs of (A) polystyrene film prepared with $3 \mathrm{wt} \%$ polystyrene in toluene, (B) cut polystyrene film. 55

Figure 3.3: (A) UV-Vis of silver nanocubes supported on polystyrene thin-films and (B) Atomic force micrograph of silver nanocubes supported on polystyrene thin-films. 56

Figure 3.4: UV-Vis of silver nanocube monolayers on polystyrene at different time intervals during the embedding process at $115.5^{\circ} \mathrm{C}$. 57

Figure 3.5: FDTD Calculated spectra of silver nanocubes with partial copper oxide shells with, $65 \mathrm{~nm}, 55 \mathrm{~nm}, 45 \mathrm{~nm}$ and $35 \mathrm{~nm}$ shell heights, measured from the bottom of the cube. 59 
Figure 3.6: Silver nanocube monolayers before (Black) and after copper oxide growth (Red) for (A) embedded (slide 14_4) and (B) supported (slide 1_3) samples.

Figure 3.7: (Left) Transmission electron micrographs of shells grown with 10 additions of hydrazine, with lactic acid from (A1-A3) embedded (slide 14_4) and (B1-B3) supported (slide 1_3) samples (Right) In-solution spectra of samples prepared from (Red) embedded (slide 14_4), (Black) supported (slide 1_3) samples

Figure 3.8: Silver nanocube monolayers before (Black) and after copper oxide growth (Red) for (A) embedded (slide 14_2) and (B) supported (slide 1_5) samples. 63

Figure 3.9: Transmission electron micrographs of shells grown with 10 additions of copper, with lactic acid from (A1-A3) embedded (slide 14_2) and (B1-B3) supported (slide 1_5) samples. (Right) In-solution spectra of samples prepared from (Red) embedded (slide 14_2), (Black) supported (slide 1_5) samples. 64

Figure 3.10: Silver nanocube monolayers before (Black) and after copper oxide growth (Red) for (A) embedded (slide 14_3) and (B) supported (slide 11_4) samples. 65

Figure 3.11: (Left) Transmission electron micrographs of shells grown with 10 additions of hydrazine, without lactic acid from (A1-A3) embedded (slide 14_3) and (B1-B3) supported (slide 11_4). (Right) In-solution spectra of 
samples prepared from (Red) embedded (slide 14_3), (Black) supported (slide 11_4) samples.

Figure 3.12: Silver nanocube monolayers before (Black) and after copper oxide growth (Red) for (A) embedded (slide 14_1) and (B) supported (slide 11_3) samples.

Figure 3.13: (Left) Transmission electron micrographs of shells grown with 10 additions of copper, without lactic acid from (A1-A3) embedded (slide 14_1) and (B1-B3) supported (slide 11_3). (Right) In-solution spectra of samples prepared from (Red) embedded (slide 14_1) and (Black) supported (slide 11_3) samples. 68

Figure 3.14: Silver nanocube monolayers before (Black) and after copper oxide growth (Red) for (A) embedded (slide 13_4) and (B) supported (slide 1_2) samples.

Figure 3.15: (Left) Transmission electron micrographs of shells grown with 10 additions of hydrazine, with lactic acid from (A1-A3) embedded (slide 13_4) and (B1-B3) supported (slide 1_2) samples, (Right) In-solution spectra of samples prepared from (Red) embedded (slide 13_4), (Black) supported (slide 1_2) samples.

Figure 3.16: Silver nanocube monolayers before (Black) and after copper oxide growth (Red) for (A) embedded (slide 13_2) and (B) supported (slide 1_4).72 Figure 3.17: Transmission electron micrographs of shells grown with 10 additions of copper, with lactic acid from (A1-A3) embedded (slide 13_2) and (B1-B3) supported (slide 1_4) samples, (Right) In-solution spectra of samples 
prepared from (Red) embedded (slide 13_2), (Black) supported (slide 1_4) samples.

Figure 3.18: Silver nanocube monolayers before (Black) and after copper oxide growth (Red) for (A) embedded (slide 13_3) and (B) supported (slide 11_2).

Figure 3.19: Transmission electron micrographs of shells grown with 10 additions of hydrazine, without lactic acid (A1-A3) embedded (slide 13_3) and (B1-B3) supported (slide 11_2) samples. (Right) In-solution spectra of samples prepared form (Red) embedded (slide 13_3), (Black) supported (slide 11_2) samples. 74

Figure 3.20: Silver nanocube monolayers before (Black) and after copper oxide growth (Red) for (A) embedded (slide 13_1) and (B) supported (slide 11_1) samples. 75

Figure 3.21: (Left) Transmission electron micrographs of shells grown with 10 additions of copper, without lactic acid from (A1-A3) embedded (slide 13_1) and (B1-B3) supported (slide 11_1) samples, (Right) In-solution spectra of samples prepared from (Red) embedded (slide 13_1), (Black) supported (slide 11_1) samples.

Figure 3.22: UV-Vis spectra of silver nanocube samples initially (Black), embedded into polystyrene (Red) and throughout growth of copper oxide shells, with four additions of hydrazine (Blue), six additions of hydrazine (Pink), eight additions of hydrazine (Green) and ten additions of hydrazine (Dark Blue). 
Figure 3.23: Silver nanocube monolayers before (Black) and after copper oxide growth (Red) for (A) embedded (slide 16_4) and (B) supported (slide 1_1) samples.

Figure 3.24: (Left) Transmission electron micrographs of shells grown with 10 additions of hydrazine (A1-A3) embedded (slide 16-4) and (B1-B3) supported (slide 1_1) samples. (Right) In-solution spectra of samples prepared from (Red) embedded (slide 16_4) and (Black) supported (slide 1_1) samples. 82

Figure 3.25: Silver nanocube monolayers before (Black) and after copper oxide growth (Red) for (A) embedded (slide 16_2) and (B) supported (slide 22_2) samples.

Figure 3.26: (Left) Transmission electron micrographs of shells grown with 10 additions of copper, with lactic acid (A1-A3) embedded (slide 16_2) and (B1B3) supported (slide 22_2) samples. (Right) In-solution spectra of samples prepared from (Red) embedded (slide 16_2), (Black) supported (slide 22_2) samples. 84

Figure 3.27: Silver nanocube monolayers before (Black) and after copper oxide growth (Red) for (A) embedded (slide 16_3) and (B) supported (slide 18_2) samples. 85

Figure 3.28: (Left) Transmission electron micrographs of shells grown with 10 additions of hydrazine, without lactic acid from (A1-A3) embedded (slide 16_3) and (B1-B3) supported (slide 18_2) samples, (Right) In-solution 
spectra of samples prepared from (Red) embedded (slide 16_3), (Black)

supported (slide 18_2) samples.

86

Figure 3.29: Silver nanocube monolayers before (Black) and after copper oxide growth (Red) for (A) embedded (slide 16_1) and (B) supported (slide 18_1) samples. 87

Figure 3.30: (Left) Transmission electron micrographs of shells grown with 10 additions of copper, without lactic acid from (A1-A3) embedded (slide 16_1) and (B1-B3) supported (slide 18_1) samples, (Right) In-solution spectra of samples prepared from (Red) embedded (slide 16_1), (Black) supported (slide 18_1) samples.

Figure 3.31: UV-Vis spectra of embedded silver nanocube monolayers before (Black) and after copper oxide growth (Red) for different added amounts of 5 $\mathrm{M} \mathrm{NaOH}$ and $0.5 \mathrm{M} \mathrm{Cu}\left(\mathrm{NO}_{3}\right)_{2}$ (A) $550 \mu \mathrm{L}$ (slide 23_2), (B) $450 \mu \mathrm{L}$ (slide 5_3), (C) $350 \mu \mathrm{L}$ (slide 5_1). 91

Figure 3.32: TEM images for structures produced from embedded nanocube monolayers with different added amounts of $5 \mathrm{M} \mathrm{NaOH}$ and $0.5 \mathrm{M} \mathrm{Cu}\left(\mathrm{NO}_{3}\right)_{2}$ (A) $550 \mu \mathrm{L}$ (slide 23_2), (B) $450 \mu \mathrm{L}$ (slide 5_3), (C) $350 \mu \mathrm{L}$ (slide 5_1)....... 92

Figure 3.33: UV-Vis spectra of embedded silver nanocube monolayers before (Black) and after copper oxide growth (Red) for different added amounts of 5 $\mathrm{M} \mathrm{NaOH}, 4 \mathrm{M}$ lactic acid and $0.5 \mathrm{M} \mathrm{Cu}\left(\mathrm{NO}_{3}\right)_{2}$ (A) $550 \mu \mathrm{L}$ (slide 23_3), (B) $450 \mu \mathrm{L}$ (slide 5_4), (C) $350 \mu \mathrm{L}$ (slide 23_1). 95

Figure 3.34: TEM images for structures produced from embedded nanocube monolayers with different added amounts of $5 \mathrm{M} \mathrm{NaOH}, 4 \mathrm{M}$ lactic acid and 
$0.5 \mathrm{M} \mathrm{Cu}\left(\mathrm{NO}_{3}\right)_{2}$ (A) $550 \mu \mathrm{L}$ (slide 23_3), (B) $450 \mu \mathrm{L}$ (slide 5_4), (C) $350 \mu \mathrm{L}$

(slide 23_1) 96 


\section{1 - Introduction}

This section will provide a description of anisotropic materials, their properties, potential applications, and synthesis methods. The production of anisotropic nanoparticles, namely anisotropic silver-copper oxide core-shell nanoparticles, is the aim of this work.

The fabrication of such structures first involves the synthesis of silver nanocubes, accomplished by the Polyol process, and subsequent growth of copper oxide shells, both of which are also described in this section. Fine control over the produced structures is desired, and so methods of shape control in particle growth are explained. In addition, the silver nanocubes are embedded in polystyrene thin films, so that the resultant surface coverage of the shell is dependent on the exposed portion. As such, a description of the Langmuir-Blodgett method to assemble the particles on the thin films and properties of the polystyrene thin films and the embedding process is given. In addition, a description of Plasmonics is given, as such properties are used to characterize the particles.

\section{1 - Anisotropic Nanomaterials}

Unlike isotropic materials, which possess consistent properties in all orientations, anisotropic materials have properties which differ when measured in different directions. Anisotropic nanomaterials include materials which possess morphological anisotropy, chemical anisotropy, or both (Figure 1.1). ${ }^{1}$ Morphological anisotropy refers to nonsymmetric structures, and includes a large variety of shaped nanoparticles, such as nanorods, ${ }^{2}$ wires, ${ }^{3}$ triangles, ${ }^{4}$ plates,${ }^{5}$ nanobars, ${ }^{6}$ nanorice ${ }^{7}$ and nanostars ${ }^{8,9}$. In contrast, chemical anisotropy refers to chemical composition which is not isotropic, including 
Janus and patchy particles. Janus particles are those in which the components are segregated to the two opposite hemispheres, while patchy particles have the components separated into distinct regions. ${ }^{10}$ Many varieties of such particles have been produced, including bimetallic nanoparticles, ${ }^{11}$ metal-metal oxide, ${ }^{12}$ metal oxide-polymer, ${ }^{13}$ polymer-polymer ${ }^{14}$ and the like.

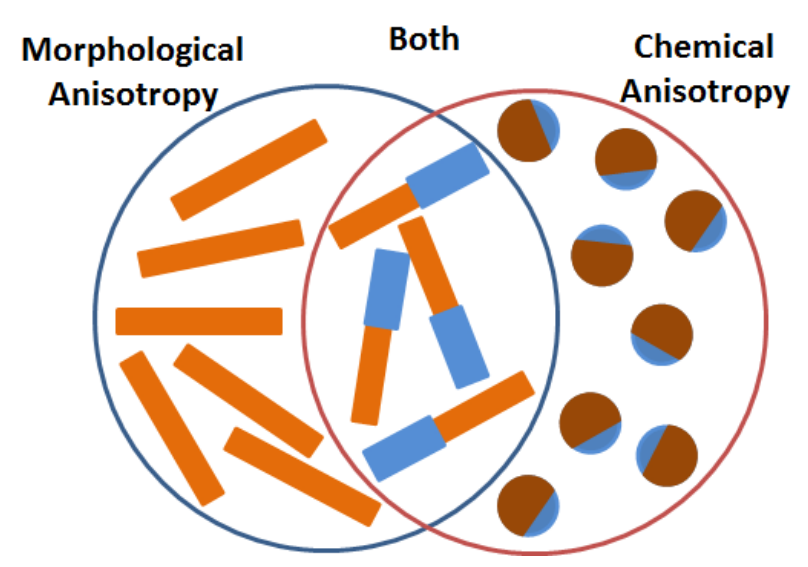

Figure 1.1: Schematic of structures with morphological anisotropy, chemical anisotropy and both morphological and chemical anisotropy, where a different colour represents a different material.

\subsection{1 - Applications and Properties of Anisotropic Materials}

Particles that exhibit anisotropy, whether chemical or morphological, have unique properties, resulting in their possible use in a multitude of applications that could not be realized with isotropic materials. Directed assembly is one such application accomplished with anisotropic materials, as opposed to isotropic materials, which lack directional interactions. ${ }^{1}$ The ability to control the orientations and the manner in which nanoparticles assemble is of paramount importance for the use of such particles. Currently, a variety of structures have been produced by making use of these anisotropic properties, including nanoparticle chains, ${ }^{15-18}$ dimers, ${ }^{19}$ and small clusters (trimer, hexamer, small ring $)^{20}$. Applications of said assemblies include sensors $^{21}$ and waveguides. $^{22,23}$ 
Gold nanorods, a well-studied structure with morphological anisotropy, are often capped with cetyltrimethylammonium bromide (CTAB). CTAB exists on the surface as a bilayer, with the ammonium head group of the first layer interacting with the anionic nanorod surface and a second layer with the ammonium head group directed towards the environment. ${ }^{24}$ Due to higher surface curvature at the nanorod's ends, CTAB coverage is reduced in these regions, making replacement of the CTAB or binding of another compound more likely than on the sides of the rod, where the CTAB is tightly packed. ${ }^{1}$ Consequently, chains of nanorods have been prepared, through the functionalization of the tips with thiolated polystyrene and the subsequent addition of a solvent that is less appropriate for polystyrene, resulting in the aggregation of the polystyrene-functionalized tips and thus the tip-to-tip assembly of the nanorods. ${ }^{25}$ Similar assembly has also been accomplished by functionalizing the nanorods' tips with biotin disulfide, with the subsequent addition of streptavidin (a protein to which biotin strongly binds) resulting in the aggregation of the rods, and again assembly in a tip-to-tip fashion. ${ }^{26}$ Chains of silver nanocubes have also been produced by similar means, and have been observed to organize into more linear structures than similarly sized silver nanospheres. ${ }^{27}$

In addition, $\mathrm{Au}-\mathrm{Fe}_{3} \mathrm{O}_{4}$ patchy particles have been used as colloidal chain stoppers, in order to control the length of assembly of gold nanorods. ${ }^{28}$ The nanorods were functionalized with thiolated polystyrene, as was the Au portion of the patchy particle. The Au portion was synthesized such that its size was complementary to that of the gold nanorod, while the $\mathrm{Fe}_{3} \mathrm{O}_{4}$ portion was larger, to impede multiple chain stoppers from attaching to the tips and to prevent side reactions. Upon introduction of these patchy particles to a solution containing the gold nanorods, they could attach to the gold 
nanorods and thus hinder other nanorods from attaching. In this way, the length of the nanorod chains could be controlled based on the reaction time before the addition of the $\mathrm{Au}-\mathrm{Fe}_{3} \mathrm{O}_{4}$ particles. $^{28}$

Directed assembly can also be accomplished with particles with chemical anisotropy, including Janus particles. Chains of Janus particles were assembled where one hemisphere was stabilized with poly(vinyl pyrrolidone) (PVP) while the other was stabilized with poly(acrylic acid) (PAA). PVP and PAA can H-bond, which resulted in the assembly of the particles into long chains. ${ }^{16}$ In addition to making use of interactions between capping (stabilizing) molecules, assembly has been accomplished by coating polystyrene spheres with a thin, partial layer of iron oxide. Upon subjecting these particles to a magnetic field, staggered chains, double chains or no chains were formed, depending on the type (oxidation state) of iron oxide deposited. ${ }^{17}$

Similar to applications in directed assembly, amphiphilic Janus particles, including polystyrene-silica ${ }^{13}$, gold-silica ${ }^{29,30}$, gold-iron oxide $^{31}$, and polystyrenepolymethyl methacrylate ${ }^{14}$ have been used as emulsifying agents for insoluble liquids. In such systems, the particles can be produced to stabilize oil-in-water or water-in-oil emulsions, where the hydrophobic portion of the particle is in the oil phase and the hydrophilic portion is in the aqueous phase. ${ }^{13}$

Additionally, particles with chemical anisotropy can have interesting applications in catalysis. Metal particles are often used as co-catalysts with semiconductor particles, improving upon the catalytic ability that either possesses alone. ${ }^{32,33}$ In photocatalysis, photons excite electron $\left(\mathrm{e}^{-}\right)$-hole $\left(\mathrm{h}^{+}\right)$pairs in the semiconductor, which can drive redox reactions at the surface. ${ }^{33}$ Core-shell geometries are frequently used in catalysis; in such 
applications, reactants must be able to get close to the surface. ${ }^{34}$ For this reason, porous shells are often used, along with particles with a partial-shell or Janus particles. Copper (I) oxide, the shell material of choice for this work, has been used for photocatalytic applications, including degradation of methyl orange ${ }^{35}$ and water splitting. ${ }^{36}$

Furthermore, metallic nanoparticles can support localized surface plasmon resonances (described in Section 1.4), which occur when the delocalized electrons in the nanostructure oscillate collectively in resonance with the incident light. ${ }^{37}$ Anisotropic nanoparticles possess unique plasmonic properties. For example, nanorods can support both a longitudinal and a transverse resonance, which can be tuned by altering the length and the width of the nanorod. ${ }^{24,38}$ Moreover, charge will accumulate in sharp corners and edges of shaped nanoparticles, which can have implications in sensing. ${ }^{39}$ Anisotropy can also result in hybridization of the plasmonic modes (described in section 1.4.5), where each plasmonic mode is directed to a different area of the particle.

\subsection{2 - Methods of Preparation for Anisotropic Materials}

Generally, since spherical or near-spherical nanoparticles are the lowest energy structure (lowest surface energy), these shapes will be formed, unless the reaction conditions are tuned to allow for the production of anisotropic particles. ${ }^{40}$ Often, anisotropic nanomaterials are produced with seed-mediated and templated synthesis methods. ${ }^{1}$ In particular, seed-mediated synthesises involve the growth on pre-existing seeds, and can be used to produce a variety of unique structures with morphological and chemical anisotropy. Seed-mediated methods can be divided into homogeneous growth and heterogeneous growth. ${ }^{39}$ Homogeneous growth refers to growth of the same material on an existing seed, and has been used to produce several morphologies, including 
wires ${ }^{41}$ octahedron, ${ }^{39}$ bipyramids, ${ }^{42}$ cubes $^{43}$ and plates ${ }^{44}$. Conversely, heterogeneous growth refers to growth of a different material on a seed, and has been used to produce alloy, ${ }^{45}$ core-shell, ${ }^{46-48}$ and Janus type nanoparticles ${ }^{32}$.

The Polyol process (described further in Section 1.2) is a seed-mediated synthesis method, in which three general types of seeds can be produced, namely single-crystalline, singly-twinned and multiply-twinned. ${ }^{39}$ First, homogeneous nucleation will occur: when the concentration of atoms reaches supersaturation, atoms cluster together to make nuclei, which grow into seeds. ${ }^{11}$ The ultimate structure of the nanoparticle is hugely influenced by the seed type, with single-crystalline seeds tending to grow into particles with cubic, spherical or cuboctahedral geometry, ${ }^{39}$ while singly- and multiply- twinned seeds tend to grow into bipyramids ${ }^{42}$ and wires ${ }^{41,49}$, respectively.

In contrast, heterogeneous nucleation results when atoms are deposited onto the surface of a previously formed seed. Heterogeneous nucleation can occur without homogeneous nucleation, since growth on pre-existing seeds is less energetically costly than forming a new solid phase, as is necessary for homogeneous nucleation. ${ }^{11}$ Furthermore, if the concentrations of atoms are high enough, both heterogeneous and homogeneous nucleation can occur concurrently; however, the nanostructures produced under these conditions often have a higher degree of polydispersity. ${ }^{11}$

Templated methods have also been used to produce anisotropic nanoparticles. In these methods, the templates can be hard materials, like porous membranes and nanoparticles, or soft materials, like micro-emulsions and micelles. ${ }^{1}$ Often, nanowires are grown in the pores of polycarbonate or alumina, with the dimensions of the wires being controllable based on pore size and reaction time. ${ }^{40}$ In another paper, gold nanorods were 
used as a template upon which polystyrene and silica were grown; subsequent etching of the gold produced hollow nanorods. ${ }^{50}$ Similarly, gold nanoparticles were grown on the surface of an octahedral nanoparticle, producing a Janus-like structure. Following the etching of the initial particle, a gold 'nanocup' was produced. ${ }^{51}$ In the case of microemulsions or micelles as soft templates, the nanoparticles are grown inside, in the area that has a higher concentration of precursor, due to differences in solubilities. ${ }^{1}$

\subsection{3 - Overview of the Current Approach to Synthesis of Anisotropic Nanoparticles}

The goal of this project was preparation of anisotropic core-shell nanoparticles. Specifically, the desired nanoparticle morphology was silver nanocubes with a partial copper oxide shell. To produce this structure, silver nanocubes were first prepared by the Polyol process and deposited onto polystyrene thin films with the Langmuir-Blodgett method. Subsequent heating of the substrates allowed the nanocubes to be embedded into the thin films to a controllable depth (Figure 1.2). This in turn enables the control of the shell surface coverage, as it grows on the exposed surface (Figure 1.2). Several different set-ups were used for the growth, and the precursor order, concentrations of reagents, and the mixing rates were varied, producing different structures. These particles could also be removed from the substrates and re-suspended by dissolving the polystyrene in toluene (Figure 1.2). Hence, through this process, a unique morphology can be produced.

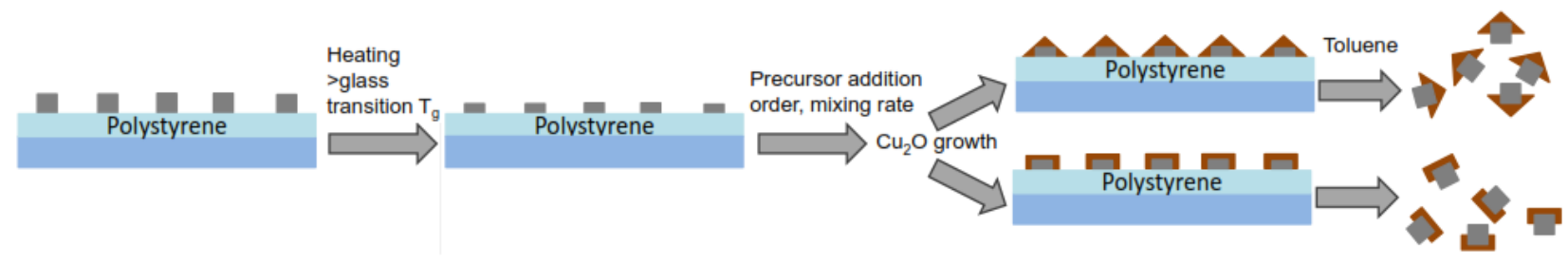

Figure 1.2: Schematic of the method of production for core-partial shell silver-copper oxide nanoparticles. 


\section{2 - Nanoparticle Synthesis}

Presently there are a wide variety of methods for the fabrication of nanoparticles. In-solution bottom-up synthesis of metal nanoparticles generally involves the reduction of metal precursors in the presence of capping agents or stabilizers, and can be used to produce an assortment of nanoparticle morphologies and compositions. ${ }^{21}$ The identity of the reducing and capping agents, as well as the temperature and relative concentrations of reactants, can greatly affect the produced structures. The Turkevitch (citrate reduction) method, ${ }^{52}$ which uses citrate as both a stabilizer and reducing agent, ${ }^{21}$ reduction by sodium borohydride ${ }^{53}$, and the Polyol process ${ }^{54}$ are all commonly employed synthesis methods for metallic nanoparticles. For this work, silver nanocubes were synthesized by the Polyol process, which allows for a high level of control over the morphology and sizes of the product.

\subsection{1 - The Polyol Process}

The Polyol process is a bottom-up method that has been used to synthesize a variety of shapes and sizes of metal nanoparticles, including wires, ${ }^{55}$ rods, ${ }^{3}$ cubes, ${ }^{43,56,57}$ bipyramids $^{42}$, and octahedra. ${ }^{43,58}$ In the Polyol process, a polyalcohol, commonly ethylene glycol, is used as the solvent and reducing agent. ${ }^{39}$ An additional molecule is used as the structural guiding and capping agent, offering increased control and tunability of the resulting structures compared to methods that use one compound as both the reducing and capping agent, such as the citrate reduction method. As such, the Polyol process is able to synthesize a greater variety of possible morphologies and allow for better shape control than these methods. The nanoparticles' ultimate shape is dependent 
on the initial seed type ${ }^{39}$ the structural guiding agent, ${ }^{59,60}$ and the presence of trace ions. $^{61,62}$

\subsection{2 - Nanoparticle Nucleation and Growth}

Prior to beginning the Polyol synthesis, ethylene glycol is heated in the presence of oxygen for at least one hour, resulting in the formation of glycolaldehyde. ${ }^{39}$ Glycolaldehyde is generally considered to be the reducing agent responsible for the Polyol process; however, ethylene glycol and poly(vinyl pyrrolidone) (PVP) (if hydroxyl terminated) can also serve as weak reducing agents. ${ }^{44,63}$ In the absence of conditions that allow for the production of glycolaldehyde, reduction of the metal precursor can still occur, but at greatly reduced rates. ${ }^{63}$

The reaction by which glycolaldehyde is produced is as follows: ${ }^{63}$

$$
2 \mathrm{HOCH}_{2} \mathrm{CH}_{2} \mathrm{CHO}+\mathrm{O}_{2} \stackrel{\Delta}{\rightarrow} 2 \mathrm{HOCH}_{2} \mathrm{CHO}+2 \mathrm{H}_{2} \mathrm{O}
$$

A typical nanoparticle synthesis can be thought of as containing stages: specifically, nucleation, evolution of these nuclei into seeds, which no longer undergo structural fluctuations, and subsequent growth into nanoparticles. ${ }^{54}$ Nanoparticle nucleation occurs when nuclei act as a template for growth, ${ }^{64}$ and involves the reduction of metal from a higher order oxidation state to a zero valent oxidation state. ${ }^{54}$ The silver precursor $\left(\mathrm{AgNO}_{3}\right)$ is injected into the pre-heated solution, with the reduction of $\mathrm{Ag}^{+}$ions resulting in nanoparticle nucleation and growth. The proposed reduction of silver ions by glycoaldehyde is:

$$
2 \mathrm{Ag}^{+}+2 \mathrm{HOCH}_{2} \mathrm{CHO} \rightarrow 2 \mathrm{Ag}^{0}+\mathrm{HOCH}_{2} \mathrm{COCOCH}_{2} \mathrm{OH}+2 \mathrm{H}^{+}
$$

However, the formation of a new solid phase (nuclei) is energetically costly, so even at the saturation concentration $\left(\mathrm{C}_{\mathrm{S}}\right)$, the metal atoms will not condense into nuclei 
spontaneously. ${ }^{65}$ Instead, the concentration of $\mathrm{Ag}^{0}$ must reach what is referred to as the critical concentration, which allows the silver to condense into nuclei. At this point, 'burst nucleation' will occur, and the precursor concentration will drop due to the formation of many nuclei. ${ }^{64}$ Once nuclei have formed, they can grow at concentrations just above $\mathrm{C}_{\mathrm{S}}$, since the process of growth on pre-existing nuclei is less energetically costly than nuclei formation (Figure 1.3) ${ }^{64,65}$ Nuclei will grow by addition of $\mathrm{Ag}^{0}$ to the nuclei surface and by coalescence of small particles into larger ones. ${ }^{64}$ Specifically, the exothermicity of lattice formation is the driving force for the formation of nuclei, with the Gibbs free energy change $(\Delta \mathrm{G})$ for nucleation being influenced by solid crystals having increased stability relative to the solvated form (decrease $\Delta \mathrm{G}$ ), while formation of solidliquid interfaces generally increases the $\Delta \mathrm{G} .{ }^{65}$

In syntheses that require the reduction of metal precursors, including the Polyol Process, clusters can also form without first being reduced to a zero valent state.$^{54}$ Due to orbital delocalization, these partially reduced clusters will have higher electron affinity, resulting in reduction occurring preferentially at these units. ${ }^{54}$ Therefore, these clusters can grow further into nanoparticles. 


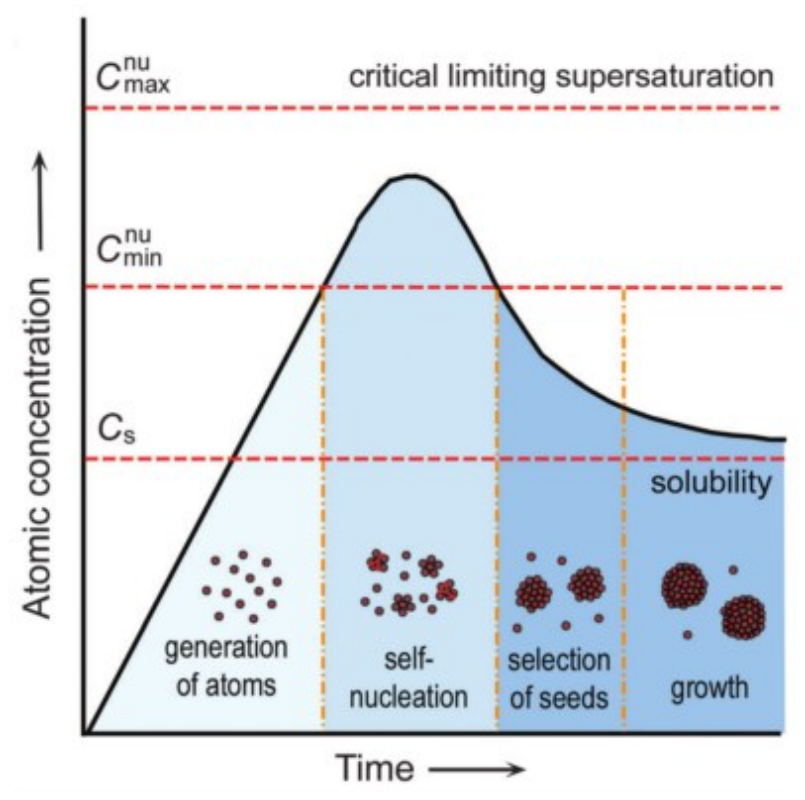

Figure 1.3: Nucleation of nanoparticles: First reduction generates zero-valent atoms, the concentration rises until it reaches the critical concentration, which allows for nucleation of nanoparticles, which can subsequently grow at concentrations lower than the critical concentration. Reprinted with permission from $\left({ }^{65}\right)$. Copyright (c) 2012, Royal Chemical Society.

As the crystallite grows it will reach a critical size, after which it has a welldefined structure (seed), as structural fluctuations become energetically costly. ${ }^{39}$ In general, the produced seeds will be of three morphologies: single-crystalline, singlytwinned and multiply twinned. Further growth on each of these seed types will tend to produce specific morphologies of products (Figure 1.4), with single-crystalline seeds being the desired seed to produce cubic nanoparticles. ${ }^{39}$ Singly- and multiply-twinned seeds can grow into other morphologies, such as right bipyramids and wires, respectively (Figure 1.4). Hence the selection of particular seeds, through oxidative etching and addition of trace amounts of sulfide, as well as other controls over product morphology, is of utmost importance, and is the topic of the following sections. Overall, the evolution of seeds into nanoparticles is due to a competition between growth and dissolution. The occurrence of Ostwald ripening can facilitate the dissolution of small seeds (often 
twinned) and growth of larger seeds (often single crystalline), narrowing the size distribution and aiding the production of cubic nanoparticles. ${ }^{65}$

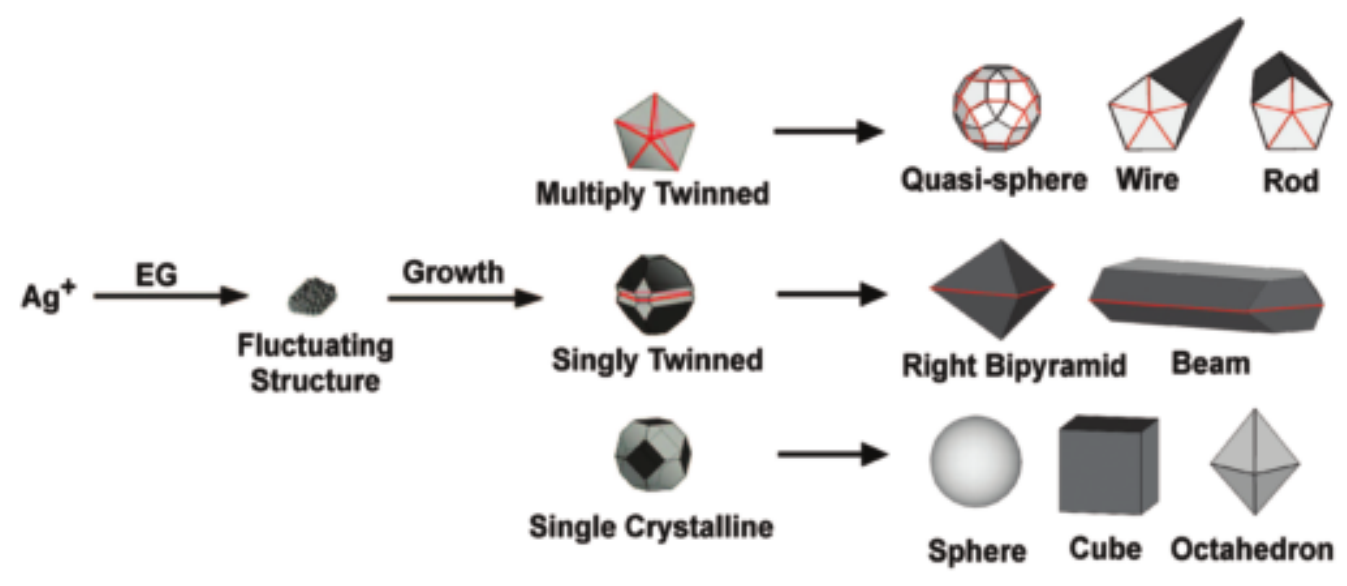

Figure 1.4: Multiply twinned, singly twinned and single crystalline seeds and produced structures. Reprinted with permission from $\left({ }^{39}\right)$. Copyright () 2011, American Chemical Society.

\subsection{3 - Control of Product Morphology: Thermodynamic vs Kinetic Products}

Control over the morphology of the produced nanostructures can be accomplished with the use of thermodynamic and kinetic parameters. ${ }^{66}$ Atoms are typically added to areas of the highest surface energy, and then surface diffusion can occur on the surface of the particle. A system is under thermodynamic control when sufficient time is given for the atoms to organize in such a way that the global minimum of Gibbs free energy of the system is obtained (Figure 1.5). In contrast, kinetically limited growth occurs if the surface diffusion is not sufficient, resulting in the nanoparticles being locked in a thermodynamically less favourable state, a local minimum (Figure 1.5). ${ }^{66}$ The type of growth is strongly affected by the ratio of atom deposition $\left(\mathrm{V}_{\text {deposition }}\right)$ to surface diffusion $\left(\mathrm{V}_{\text {diffusion }}\right)$, and is considered to be under thermodynamic control if $\mathrm{V}_{\text {deposition }}<\mathrm{V}_{\text {diffusion }}$ and under kinetic control if $\mathrm{V}_{\text {deposition }}>\mathrm{V}_{\text {diffusion. }}{ }^{66}$ 


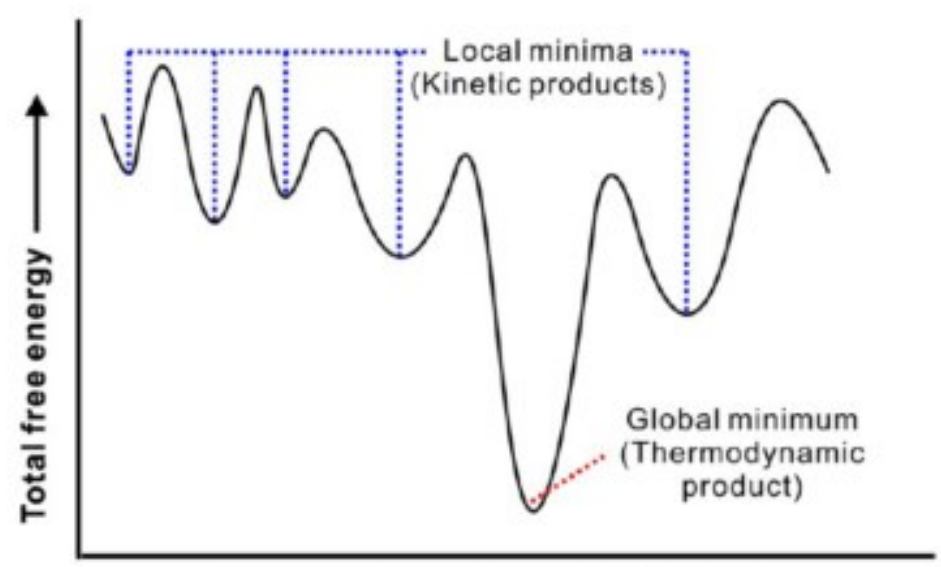

Parameter representing structure

Figure 1.5: Total free energy of products vs parameters representing structures, indicating for different reaction conditions formation of kinetic products (local minimum) and the thermodynamic product (global minimum). Reprinted with permission from $\left({ }^{66}\right)$. Copyright $(\odot)$ 2015, American Chemical Society.

\subsection{4 - Thermodynamic Control Over Product Morphology}

Thermodynamic control over product morphology can be accomplished by introducing structural guiding agents that can alter the relative interfacial energy of the facets. The interfacial energy $(\gamma)$ (surface free energy) for an ideal (atomically flat) surface is expressed by: $:^{54,66}$

\section{Equation 1}

$$
\gamma=\frac{1}{2} N_{b} \varepsilon \rho_{a}
$$

where $\mathrm{N}_{\mathrm{b}}$ is the number of broken bonds (from forming the new surface), $\varepsilon$ is the bond strength and $\rho_{\mathrm{a}}$ is the surface density.

The interfacial energy varies between different crystallographic facets, since the surface density and the number of broken bonds differ between facets. For silver and other fcc structures, the interfacial energy follows the sequence $\gamma_{\{111\}}<\gamma_{\{100\}}<\gamma_{\{110\}}{ }^{54,66}$ Nanoparticles will tend to produce the lowest energy facets, so silver nanoparticles will typically be capped with $\{111\}$ and $\{100\}$ facets. However, the introduction of a 
compound that attaches preferentially to a specific facet can serve to stabilize this facet and lower its interfacial energy, which allows this facet to be expressed on the nanoparticle. $^{66}$

Poly(vinyl pyrrolidone) is commonly used as a capping agent in the Polyol process because it is able to support the growth of a variety of shapes of nanoparticles. ${ }^{60}$ Poly(vinyl pyrrolidone) physisorbs to the surface of the silver nanoparticles through pyrrolidone's nitrogen and oxygen unpaired electrons. ${ }^{66,67}$ The binding affinity varies for different facets,${ }^{54}$ with PVP having a greater affinity for silver's $\{100\}$ facet. ${ }^{41}$ As a result, PVP stabilizes this facet, and slows the addition of silver atoms onto the $\{100\}$ face by steric hinderance. ${ }^{54}$ Due to this, the other facets can grow more rapidly, until the particle is completely capped by $\{100\}$ facets. At this point, the nanoparticle can uniformly increase in size.

\subsection{5 - Kinetic Control Over Product Morphology}

Kinetic control over the product morphology can be accomplished by changing the rate of reduction of precursors, or oxidative etching of the particles. ${ }^{66}$ In the Polyol synthesis, trace amounts of sulfide $\left(\mathrm{S}^{-}\right)$or hydrosulfide $\left(\mathrm{HS}^{-}\right)$are often added to increase the rate of reduction of $\mathrm{AgNO}_{3}$. This increased reduction rate results because, immediately after the addition of $\mathrm{AgNO}_{3}$ to a solution containing sulfide ions, silver sulfide $\left(\mathrm{Ag}_{2} \mathrm{~S}\right)$ nanocrystallites form. ${ }^{39}$ These crystallites can serve as a primary site for nucleation and can catalyze the reduction of $\mathrm{Ag}^{+}$, leading to nanoparticle growth. In particular, the formation of $\mathrm{Ag}_{2} \mathrm{~S}$ decreases the reduction potential of $\mathrm{Ag}^{+}$relative to free $\mathrm{Ag}^{+}{ }^{56}$ This is very beneficial in the synthesis, as the nucleation event occurs more quickly, aiding the production of nanoparticles with a consistent size. ${ }^{56}$ In addition, 
increasing the rate of nucleation and growth will tend to produce single-crystalline seeds, which are necessary to grow into nanoparticles with a cubic geometry. Twin defects, which occur when a layer of atoms acts as a (111) mirror plane, induce strain on the nanoparticles, which increases as the size increases. ${ }^{68}$ As such, in a synthesis where seeds grow rapidly, the produced seeds will generally be single-crystalline, as the strain of twinned and multiply-twinned seeds makes their production less favourable.

In addition, control over the particles' morphology can be accomplished via oxidative etching, wherein the zero valent metal ions are oxidized back to ions. This is accomplished by adding trace amounts of specific ions, often $\mathrm{Cl}^{-}$or $\mathrm{Br}^{-}$, which, in the presence of oxygen from air, can oxidatively etch the growing silver seed. ${ }^{39}$ Twin boundaries (in both multiply-twinned and singly-twinned seeds), result in lattice distortion and strain, which make these seeds more prone to oxidative etching. ${ }^{61}$ This is useful in the synthesis of silver nanocubes, since of the three seed types produced (singlecrystalline, singly-twinned, multiply-twinned), only single-crystalline seeds tend to grow into nanoparticles with cubic geometry. Single crystalline seeds can be selected since the defect regions of twinned seeds are at a higher energy than single crystalline seeds, making them more susceptible to oxidative etching. ${ }^{54}$

\section{3 - Anisotropic Copper Oxide Shells on Silver Nanocubes}

Anisotropic nanoparticles have unique properties and can be potentially useful for directed self-assembly, preparation of nanoparticle dimers, sensing and catalysis. In this work, copper oxide is grown on the exposed portions of silver nanocubes, which have been partially embedded in polystyrene thin films. These particles can subsequently be removed from the surface, producing anisotropic nanoparticles. 


\subsection{1 - Copper Oxide}

Copper (I) oxide $\left(\mathrm{Cu}_{2} \mathrm{O}\right)$ is a p-type semiconductor with a direct band gap of $\sim 2.2$ $\mathrm{eV} \cdot{ }^{69} \mathrm{Cu}_{2} \mathrm{O}$ crystalizes in a cubic structure, with oxygen arranged in a body-centered cubic arrangement, and copper arranged tetrahedrally around each oxygen atom (Figure 1.6, left). ${ }^{70}$ The different atomic surface identities of the facets results in a change in surface chemistries, and therefore a difference performance in many properties and applications, including catalysis,${ }^{35}$ sensing, ${ }^{71}$ electrical properties ${ }^{70}$ and antibacterial properties ${ }^{72} \cdot{ }^{70}$ In particular, the three low-index facets, $\{100\},\{111\}$ and $\{110\}$, often denoted $\mathrm{c}-\mathrm{Cu}_{2} \mathrm{O}, \mathrm{o}-\mathrm{Cu}_{2} \mathrm{O}$ and $\mathrm{d}-\mathrm{Cu}_{2} \mathrm{O}$, respectively, each have different surface termination. $^{70}$

The $\{100\}$ facet alternates termination with $\mathrm{Cu}^{+}$and $\mathrm{O}^{2-}$ layers (Figure 1.6a), so an ideal $\{100\}$ is composed exclusively of either oxygen or copper atoms. ${ }^{73}$ The $\{111\}$ facet possesses hexagonal symmetry, and repeats as a three-plane unit, with each coppercontaining plane between two oxygen-containing planes, with the ideal facet being terminated by an outer layer of oxygen anions, a layer of copper cations and finally another layer of oxygen anions (Figure 1.6c). ${ }^{72}$ In this facet, every second surface copper ion has a dangling bond. These three layers are often approximated as being in the same surface, since the distance between the layers is very small. ${ }^{72}$ The $\{110\}$ direction alternates between layers of copper cations and copper-oxygen mixed layers (Figure 1.6b). ${ }^{74}$ This facet has $\sim 1.5 \times$ more copper cations per unit area than the $\{111\}$ facet. ${ }^{35}$ Since the synthesis is usually carried out in aqueous medium, oxygen termination (or copper-oxygen termination for $\{110\})$ is expected, as copper termination would be unstable due to the reaction with the $\mathrm{OH}$ in water. ${ }^{72,75}$ For these facets of $\mathrm{Cu}_{2} \mathrm{O}$, the 
surface energies follow the sequence $\gamma_{\{100\}}<\gamma_{\{111\}}<\gamma_{\{110\}}$, which is related to the density of copper dangling bonds. ${ }^{70}$

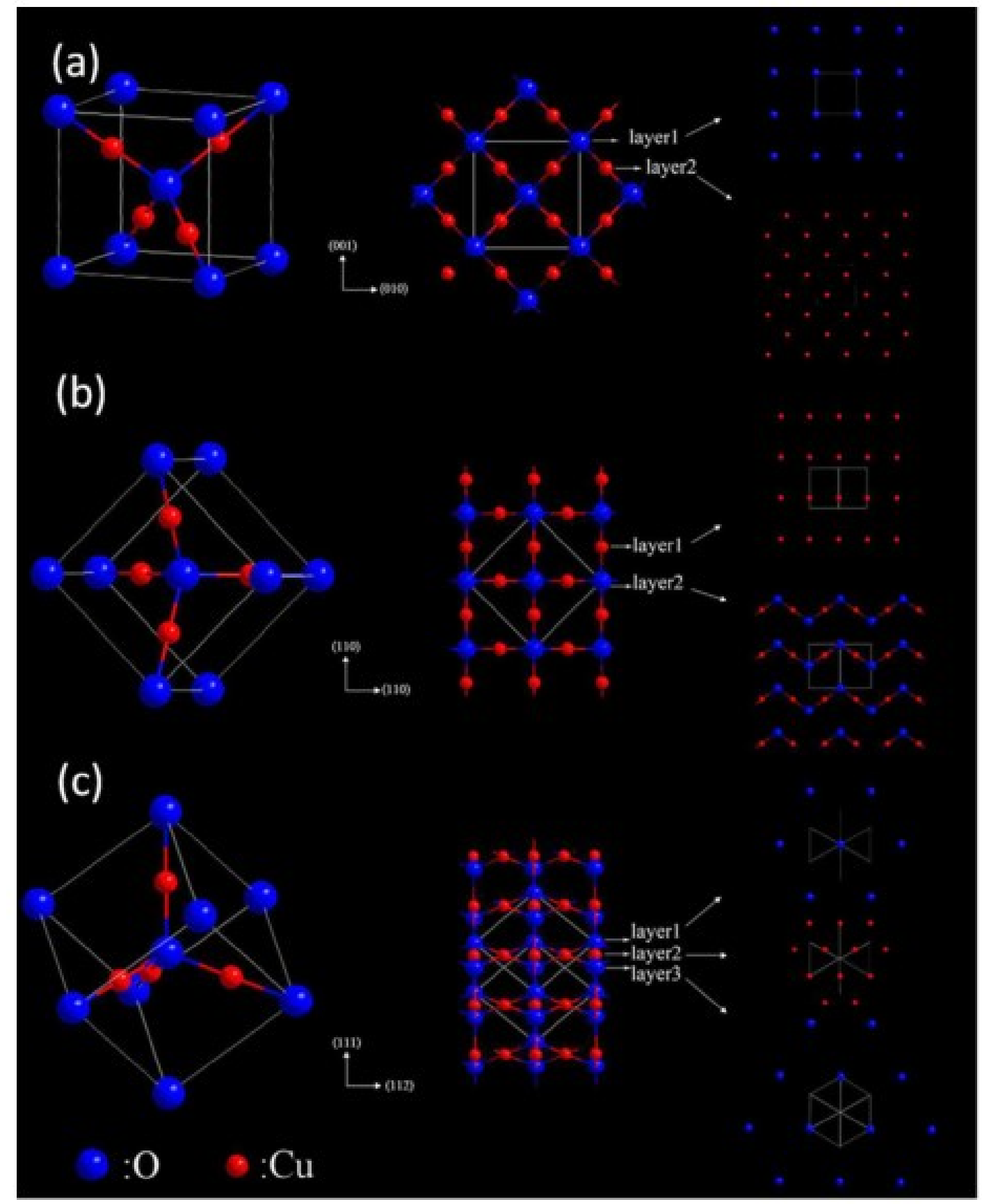

Figure 1.6: $\mathrm{Cu}_{2} \mathrm{O}$ crystal structure and structures of (a) (100), (b) (110) and (c) (111) crystal facets. Reprinted with permission from $\left({ }^{74}\right)$. Copyright $\odot 2015$, Elsevier. 


\subsection{2 - Copper Oxide Growth}

Production of nanomaterials first involves reactions between the precursors, generating monomers, which allows for nucleation and then growth of the material. ${ }^{68}$ In general, it is desirable to produce structures with controlled sizes and shapes. Control can be accomplished with the use of compounds which preferentially adsorb to a specific facet, ${ }^{70}$ and by adjusting the ratios ${ }^{76}$ and the order of addition ${ }^{68}$ of precursors.

Often, $\mathrm{Cu}_{2} \mathrm{O}$ is often grown in solution with the use of a copper precursor (such as $\left.\mathrm{CuSO}_{4}, \mathrm{Cu}\left(\mathrm{NO}_{3}\right)_{2}\right)$, sodium hydroxide $(\mathrm{NaOH})$ and hydrazine $\left(\mathrm{N}_{2} \mathrm{H}_{4}\right)$. With the addition of $\mathrm{NaOH}$ to a solution containing the copper precursor the formation of a copper(II) hydroxide $\left(\mathrm{Cu}(\mathrm{OH})_{2}\right)$ precipitate will occur $\left(\mathrm{K}_{\mathrm{sp}}=1.6 \times 10^{-19}\right)$, and can be observed as a bright blue colour. ${ }^{77}$ Specifically: ${ }^{78}$

$$
\mathrm{Cu}^{2+}+2 \mathrm{NaOH} \rightarrow \mathrm{Cu}(\mathrm{OH})_{2}+2 \mathrm{Na}^{+}
$$

Successive reduction of $\mathrm{Cu}(\mathrm{OH})_{2}$ by $\mathrm{N}_{2} \mathrm{H}_{4}$ results in the formation of $\mathrm{Cu}_{2} \mathrm{O}$; the expected reaction can be expressed by: ${ }^{77,78}$

$$
4 \mathrm{Cu}(\mathrm{OH})_{2}+\mathrm{N}_{2} \mathrm{H}_{4} \rightarrow 2 \mathrm{Cu}_{2} \mathrm{O}+\mathrm{N}_{2}+6 \mathrm{H}_{2} \mathrm{O}
$$

In addition, the $\mathrm{Cu}(\mathrm{OH})_{2}$ can form $\mathrm{Cu}(\mathrm{OH})_{4}{ }^{2-}$, by coordinating with additional $\mathrm{OH}^{-},{ }^{79}$ which can also be reduced with hydrazine to form $\mathrm{Cu}_{2} \mathrm{O}$, according to: ${ }^{77}$

$$
\begin{gathered}
\mathrm{Cu}(\mathrm{OH})_{2}+2 \mathrm{OH}^{-} \rightleftarrows \mathrm{Cu}(\mathrm{OH})_{4}{ }^{2-} \\
4 \mathrm{Cu}(\mathrm{OH})_{4}{ }^{2-}+\mathrm{N}_{2} \mathrm{H}_{4} \rightarrow 2 \mathrm{Cu}_{2} \mathrm{O}+\mathrm{N}_{2}+6 \mathrm{H}_{2} \mathrm{O}+8 \mathrm{OH}^{-}
\end{gathered}
$$

However, many reducing agents other than hydrazine have been employed, including ascorbic acid, ${ }^{68}$ sodium ascorbate, ${ }^{80}$ formic acid ${ }^{81}$ glucose ${ }^{79}$ and hydroxyl amine $^{82}$. In addition, many compounds have been used as capping or structural guiding agents, including EDTA, ${ }^{83} \mathrm{I}^{-84} \mathrm{Cl}^{-79}, \mathrm{PVP}^{85}{ }^{8 i t r a t e}{ }^{73}, \mathrm{SDS}^{86}, \mathrm{CTAB}^{80}, \mathrm{PEG}^{87}, \mathrm{NH}_{3}{ }^{76}$ and 
lactate $^{74}$. The sizes produced can be adjusted by increasing the ratio of $\mathrm{OH}^{-}: \mathrm{Cu}^{2+}$, with increased $\mathrm{OH}^{-}$resulting in larger sizes. ${ }^{76,77}$ Similarly, increasing the amount of copper precursor added relative to the amount of silver nanoparticles resulted in thicker shells in the production of core-shell nanoparticles. ${ }^{48}$ Shape can be controlled by adding certain structural guiding agents, which stabilize and slow the growth on certain facets, ${ }^{68}$ or by adding a certain ratio of compounds that preferentially stabilize different facets, to tune the degree that each facet is expressed. ${ }^{73}$ In addition, the order that the precursors are added can affect the produced morphology. For example, in the synthesis of $\mathrm{Cu}_{2} \mathrm{O}$ in PEG with ascorbic acid and $\mathrm{NaOH}$, it was found that different structures resulted depending on the addition order of ascorbic acid and $\mathrm{NaOH}$, which was attributed to formation of different species or species ratios $\left(\mathrm{Cu}(\mathrm{OH})_{4}{ }^{2-}, \mathrm{Cu}(\mathrm{OH})_{2}\right.$, ascorbate anions), which can change the kinetics or mechanism of nanocrystal formation. ${ }^{68}$

Compounds other than $\mathrm{OH}^{-}$can complex with $\mathrm{Cu}^{2+}$; for example, lactic acid is often used in the electrochemical deposition of $\mathrm{Cu}_{2} \mathrm{O}$, where lactate will form a complex with $\mathrm{Cu}^{2+}$ in solution. ${ }^{88,89}$ The $\mathrm{Cu}^{2+}$ and lactate $\left(\mathrm{CH}_{3} \mathrm{CHOHCOO}^{-}\right)$complex ${ }^{89}$ can be reduced, forming a $\mathrm{Cu}^{+}$complex, as: ${ }^{88}$

$$
\mathrm{Cu}\left(\mathrm{CH}_{3} \mathrm{CHOHCOO}\right)_{2}+e^{-} \rightarrow \mathrm{Cu}\left(\mathrm{CH}_{3} \mathrm{CHOHCOO}\right)+\mathrm{CH}_{3} \mathrm{CHOHCOO}^{-}
$$

Copper oxide can then be formed, by: ${ }^{88}$

$$
2 \mathrm{Cu}\left(\mathrm{CH}_{3} \mathrm{CHOHCOO}\right)+2 \mathrm{OH}^{-} \rightarrow \mathrm{Cu}_{2} \mathrm{O}+\mathrm{H}_{2} \mathrm{O}+2 \mathrm{CH}_{3} \mathrm{CHOHCOO}^{-}
$$

It has been reported that at increasing $\mathrm{pH}$ lactate adsorbs preferentially to the (100), (110) and (111) facets, respectively, which can affect the produced facets. ${ }^{74}$ 


\subsection{3 - Core-shell Nanoparticles}

In the current work, in order to produce the desired structures, copper oxide must be grown on the surface of the silver nanocubes. The lattice mismatch between $\mathrm{Cu}_{2} \mathrm{O}$ and Ag is $4.2 \% .{ }^{90}$ In general, a smaller lattice mismatch is better for epitaxial growth; ${ }^{90}$ however, epitaxial growth to form core-shell structures has been accomplished with materials with larger mismatches, including $\mathrm{Au}-\mathrm{Cu}(11.4 \% \text { mismatch })^{91}$ and $\mathrm{Au}-\mathrm{Ni}$ $\left(13.6 \%\right.$ mismatch) ${ }^{92}$ Heterogeneous nucleation, nucleation on an existing surface of a different identity, must occur for the formation of core-shell particles. Following nucleation, the growth can proceed epitaxially if the lattice mismatch is sufficiently small and the conditions are appropriate. However, non-epitaxial growth can occur with a large lattice mismatch and inappropriate conditions, resulting in structures with morphologies different from the initial seeds. ${ }^{39}$ Heterogeneous nucleation, and growth, can occur at concentrations lower than would be necessary for nucleation in solution, since growth on a pre-existing structure is more favourable. ${ }^{11}$ If the concentrations are increased such that nucleation occurs in solution and on the substrate, the result is often less control over the resulting structures. ${ }^{11}$

In this work, the desired structure contains a partial copper oxide shell, which is grown on nanocubes that have been embedded into polymer films. In addition to growth on the surface of the silver, copper oxide can also grow in solution and on the polystyrene, which is porous. Growth in solution and on the substrate is ideally minimized, as this would decrease the control over the produced structures. 
The plasmonic properties of core-shell nanostructures are dependent on the morphology of the core, the thickness, porosity and coverage of the shell, and spacing between the core and shell. ${ }^{90}$ These properties are described in the following sections.

\section{4 - Plasmonics}

Plasmonics is the field of research which investigates the interaction of light with delocalized electrons in metal nanostructures and the manipulation of light on the nanoscale. $^{21,39,93}$ The production of a surface plasmon resonance (SPR), which results from the coupling of light's electric field component to a metal particle, is a requirement of plasmonics. The SPR is affected by the composition, ${ }^{94}$ shape, ${ }^{95}$ size $^{96}$ and environment ${ }^{97,98}$ of the nanostructure and, through tuning these parameters, light can be manipulated in unique ways. ${ }^{39,99}$

\subsection{1 - Surface Plasmon Resonance and Localized Surface Plasmon Resonance}

The collective oscillation of the conduction electrons in a metal can be induced by the time-varying electric field associated with light. At certain excitation frequencies, this oscillation can be in resonance with light, resulting in a surface plasmon resonance, which is a strong absorption of incident light. ${ }^{39}$ In a bulk metal, this collective oscillation propagates along the surface of the material (Figure 1.7a). However, in particles that are smaller than the wavelength of the incident light, these oscillations will be confined to the surface of the particle, resulting in dipolar and higher-order modes. ${ }^{39,100}$ For example, in a dipolar mode, the electric field forces the electrons to one side of the particle, switching directions with the variations in the electric field (Figure 1.7b). ${ }^{39}$ In the case that these oscillations are in resonance with light, a localized surface plasmon resonance (LSPR) is produced. 
a

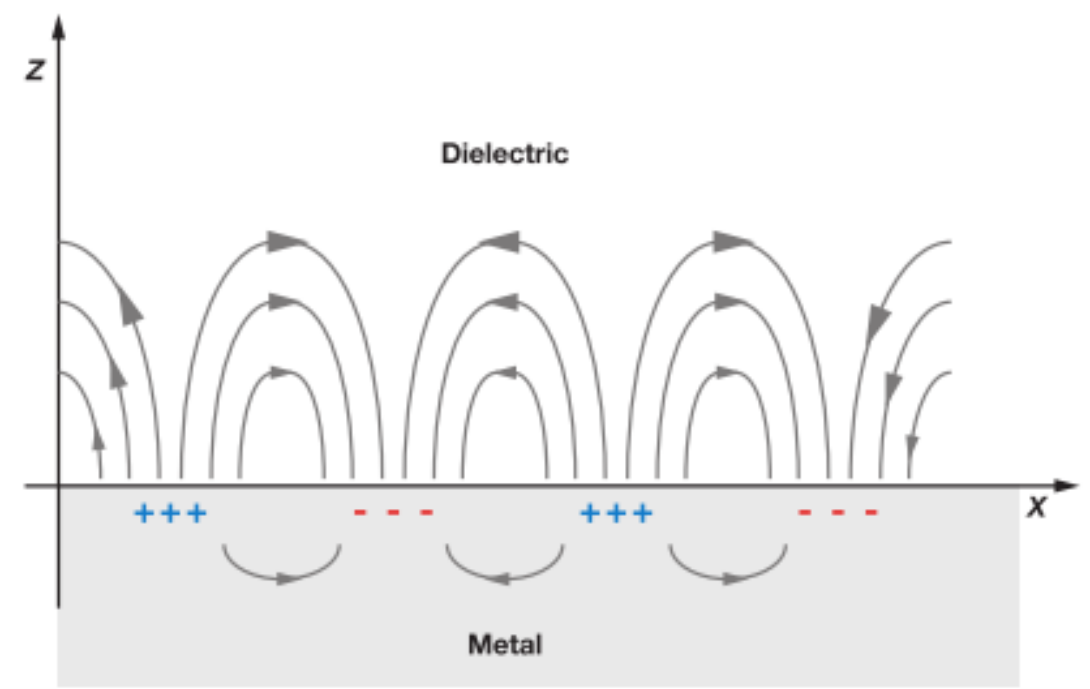

b

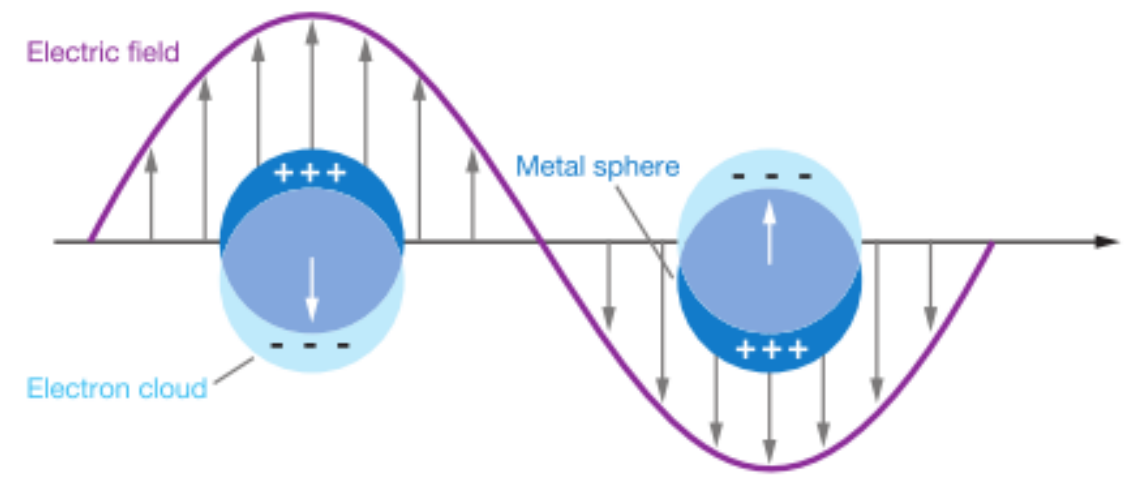

Figure 1.7: Schematic of (a) surface plasmon in a bulk metal and (b) localized surface plasmon resonance (LSPR) in a metal sphere. Reprinted with permission from $\left({ }^{37}\right)$. Copyright $($ C 2007, Annual Reviews.

\subsection{2 - Plasmonic Materials}

Silver is a material that is widely used in plasmonics, as it can support a strong surface plasmon (SP) that can be tuned across the spectrum from $300 \mathrm{~nm}$ to $1200 \mathrm{~nm} .^{39}$ This is very valuable, since it is necessary to choose a material that can support a SP at the desired resonance frequency, making silver an appropriate material to use for a variety of applications. ${ }^{23,39}$ The dielectric function $(\varepsilon)$ of a certain material reflects the interaction of that material's electrons with light, hence its ability to support a SP. The 
dielectric function is composed of a real and an imaginary part, which is different for different materials.

The extinction $\left(\sigma_{\text {ext }}\right)$ is the sum of the absorption and scattering of an electromagnetic wave as it passes through a sample, and can be expressed as the sum of the response of a single nanoparticle multiplied by the nanoparticle concentration, speficially: ${ }^{101}$

\section{Equation 2}

$$
\sigma_{\text {ext }}=\mathcal{N}\left(C_{a b s}+C_{s c a}\right)
$$

where $\mathcal{N}$ is the particle concentration, $\mathrm{C}_{\text {abs }}$ is the absorption cross-section and $\mathrm{C}_{\text {sca }}$ is the scattering cross-section.

Mie theory, which can be used describe the LSPR of a metal nanosphere, makes use of the assumptions that the spherical particle is non-interacting (in a dilute solution or well separated), and that both the particle and its surroundings are homogeneous and can be expressed with bulk dielectric constants. ${ }^{9939,102}$ A relationship for the extinction crosssection $\left(\mathrm{C}_{\mathrm{ext}}\right)$, which is the sum of the absorption and scattering cross-sections, can be expressed by solving Maxwell's equations. ${ }^{99}$ For a metal nanosphere, the extinction cross-section can be expressed by: ${ }^{39,102}$

Equation 3

$$
C_{\text {ext }}=\frac{24 \pi^{2} R^{2} \varepsilon_{m}^{\frac{3}{2}}}{\lambda}\left[\frac{\varepsilon_{i}}{\left(\varepsilon_{r}+2 \varepsilon_{m}\right)^{2}+\varepsilon_{i}^{2}}\right]
$$

where $\mathrm{R}$ is the radius of the particle, $\varepsilon_{\mathrm{m}}$ is the relative dielectric constant of the surroundings, $\varepsilon_{\mathrm{r}}$ and $\varepsilon_{\mathrm{i}}$ are the real and imaginary part of the dielectric function, 
respectively, and $\lambda$ is the wavelength of light. Both the real and imaginary part of the dielectric function are dependent on the wavelength of the incident light. ${ }^{39}$

At the resonance condition, the extinction cross-section becomes exceedingly large, and the surface plasmon absorption band appears. ${ }^{39,99}$ This condition is met when $\varepsilon_{\mathrm{r}}$ approaches $-2 \varepsilon_{\mathrm{m}}$ and $\varepsilon_{\mathrm{i}}$ approaches 0 .

The strength of the surface plasmon is of paramount importance when selecting a specific material. The quality factor (Q) is proportional to the intensity of a surface plasmon, and can be described by: $:^{39}$

\section{Equation 4}

$$
Q=\frac{\omega\left(\frac{d \varepsilon_{\mathrm{r}}}{d \omega}\right)}{2\left(\varepsilon_{\mathrm{i}}\right)^{2}}
$$

where $\omega$ is the angular frequency of light, and $\varepsilon_{\mathrm{r}}$ and $\varepsilon_{\mathrm{i}}$ are the real and imaginary parts of the dielectric function, respectively. Silver has a large quality factor over a wide range of the spectrum $(300 \mathrm{~nm}-1200 \mathrm{~nm})$, signifying that, over this region, it can support strong plasmons. ${ }^{39}$

\subsection{3 - Size and Shape Dependency}

The size and shape of the nanoparticle greatly affects its plasmonic properties. As the size of the nanoparticle increases, the LSPR red-shifts and broadens. The oscillation of electrons occurs at a lower energy for larger particles, since the size is increased relative to the incident wavelength, increasing the depolarization of the electron cloud. ${ }^{39}$ Due to this, the electrons lose energy and experience a damping effect, making the SPR broader and red-shifted. ${ }^{101}$ In addition to the shift in LSPR, as nanoparticle size increases higher order modes are produced, which have distinct electron charge distribution. For 
example, small nanoparticles support dipole modes, and as the size is increased they can support quadruple modes as well. ${ }^{39,103}$

In addition to nanoparticle size, shape has a great effect on the LSPR. In small spherical nanoparticles, the electron displacement is homogeneous, resulting in dipolar resonances, ${ }^{101}$ while non-spherical nanoparticles have LSPRs which are unevenly distributed, resulting in unique absorption properties for differently-shaped particles. ${ }^{99} \mathrm{In}$ particular, sharp features, including corners and edges, leads to an uneven distribution of charge around the nanoparticle, resulting in higher order modes in smaller particles. This charge accumulation results in high electric field intensity in these regions. ${ }^{39}$ Structures with morphological anisotropy can support additional plasmon modes; for example, gold nanorods can support a longitudinal and a transverse resonance, in contrast to their spherical counterparts. ${ }^{1}$

\subsection{4 - Environmental Effect on Localized Surface Plasmon Resonances}

The environment, including the refractive index of the surroundings, can affect the LSPR in different ways. The energy of a bulk plasmon, noting that a plasmon can be described as a quantum of plasma oscillation, can be expressed as: ${ }^{104}$

Equation 5

$$
E_{P}=\hbar \sqrt{\frac{n e^{2}}{m \varepsilon_{0}}}
$$

where $E_{p}$ is the energy of the plasmon oscillation, $h$ is the Dirac constant $(h / 2 \pi), n$ is the electron density, $\mathrm{e}$ is the charge of an electron, $\mathrm{m}$ is the mass of an electron, and $\varepsilon_{0}$ is the permittivity of free space. 
At resonance, the frequency, $\omega_{\mathrm{p}}$, is:

Equation 6

$$
\omega_{P}=\sqrt{\frac{n e^{2}}{m \varepsilon_{0}}}
$$

With the use of the Drude model, $\varepsilon_{\mathrm{r}}$ can be described as: ${ }^{104,105}$

Equation 7

$$
\varepsilon_{r}=1-\frac{\omega_{P}^{2}}{\omega^{2}+\gamma^{2}}
$$

With $\omega_{\mathrm{p}}$ being the plasmon frequency of the bulk metal, $\omega$, the frequency of incident radiation, and $\gamma$, the damping frequency of the bulk metal. However, $\gamma<<\omega$ for the visible and ultraviolet regions of the spectrum, which allows simplification to: ${ }^{104,105}$

Equation 8

$$
\varepsilon_{r}=1-\frac{\omega_{P}^{2}}{\omega^{2}}
$$

At resonance, with the condition that $\varepsilon_{\mathrm{r}}=-2 \varepsilon_{\mathrm{m}}$ :

Equation 9

$$
\varepsilon_{r}=-2 \varepsilon_{m}=1-\frac{\omega_{P}^{2}}{\omega^{2}}
$$

This can be expressed in terms of the frequency of incident radiation, specifically: ${ }^{104,105}$

Equation 10

$$
\omega=\frac{\omega_{P}}{\sqrt{2 \varepsilon_{m}+1}}
$$


This equation can be expressed in terms of wavelength $(\lambda=2 \pi \mathrm{c} / \omega)$ and refractive index $\left(\varepsilon_{\mathrm{m}}=\mathrm{n}_{\mathrm{m}}{ }^{2}\right)$, which gives the result: ${ }^{104}$

Equation 11

$$
\lambda_{\max }=\lambda_{P} \sqrt{2 n_{m}^{2}+1}
$$

with $\lambda_{\max }$ being the wavelength of the LSPR and $\lambda_{\mathrm{p}}$, the wavelength consequent of the plasma frequency of the bulk material. Through the use of this equation, the effect of the refractive index on the LSPR wavelength can be predicted. Specifically, increasing the refractive index of the surroundings results in the LSPR shifting to a longer wavelength. The relationship between LSPR wavelength and $\mathrm{n}_{\mathrm{m}}$ is approximately linear over small ranges of $\mathrm{n}_{\mathrm{m}}{ }^{104}$

\subsection{5 - Substrate-Induced Hybridization}

Plasmonic nanoparticles are sensitive to their environment, and will therefore have different plasmonic properties when suspended on a substrate compared to those in solution. In the case of a spherical nanoparticle in an isotropic environment, there are three degenerate dipolar modes. ${ }^{106}$ However, once the nanoparticle is deposited onto a dielectric substrate, the presence of the substrate results in symmetry-breaking, which can change the degeneracies of the plasmonic modes. For a spherical nanoparticle deposited on a dielectric substrate, the reduction of symmetry results in splitting these modes into two resonances, parallel and perpendicular to the dielectric layer. ${ }^{106}$ This splitting is strongly affected by the permittivity of the substrate. In particular, the nanoparticle couples to its image of surface charges (induced charges in the dielectric), screened by the factor $\left(\varepsilon_{\mathrm{s}}-1\right) /\left(\varepsilon_{\mathrm{s}}+1\right)$, with $\varepsilon_{\mathrm{s}}$ being the permittivity of the substrate. ${ }^{106}$ 
This substrate-induced hybridization is particularly large for particle geometries with a greater contact area with the substrate, such as in the case of silver nanocubes. ${ }^{97}$ For a silver nanocube, the modes most pertinent to this interaction are the intrinsic dipolar $\left(\mathrm{D}^{0}\right)$ and quadrupolar $\left(\mathrm{Q}^{0}\right)$ modes. The $\mathrm{D}^{0}$ mode is a bright mode, meaning it possesses a dipole moment which can be excited by light, and observed in the plasmonic spectrum. The induced charges for the $\mathrm{D}^{0}$ mode are largely located at the corners of the cube and, in a charge map, the same sign exists on the same side (such that overall it has a dipole) (Figure 1.8). In contrast, the $\mathrm{Q}^{0}$ mode has the charges again concentrated at the corners of the cube, but in a fashion where the overall dipole disappears (Figure 1.8). As such the $\mathrm{Q}^{0}$ mode cannot directly couple to light, and is a dark mode. ${ }^{97}$

The $\mathrm{D}^{0}$ and $\mathrm{Q}^{0}$ have charge distributions on their bottom surface which are sufficiently similar for the induced charges in the substrate to facilitate an interaction. This produces hybridized modes, which are analogous to bonding and antibonding orbitals; specifically, the 'bonding' $\left(\mathrm{D}^{0}+\mathrm{Q}^{0}\right)$ and 'antibonding' $\left(\mathrm{D}^{0}-\mathrm{Q}^{0}\right)$ modes. ${ }^{97}$ In these new hybridized modes, the 'bonding' mode is derived mostly from the intrinsic dipolar mode, and is referred to as the hybridized dipolar mode (D), while the 'antibonding' mode is mostly derived from the intrinsic quadrupolar mode, and is referred to as the hybridized quadrupolar mode (Q) (Figure 1.8). These hybridized modes are spatially separated, with the D mode having most of its charge localized toward the substrate (high refractive index), while the Q mode's charge is localized away from the substrate. ${ }^{97}$ Hence, the particle senses two environments, the substrate and the superstrate, simultaneously. This is of particular use as it allows monitoring of a nanoparticle's 
environment, which is explored further in this work through embedding silver nanocubes into polystyrene thin-films and growth of anisotropic copper oxide shells.

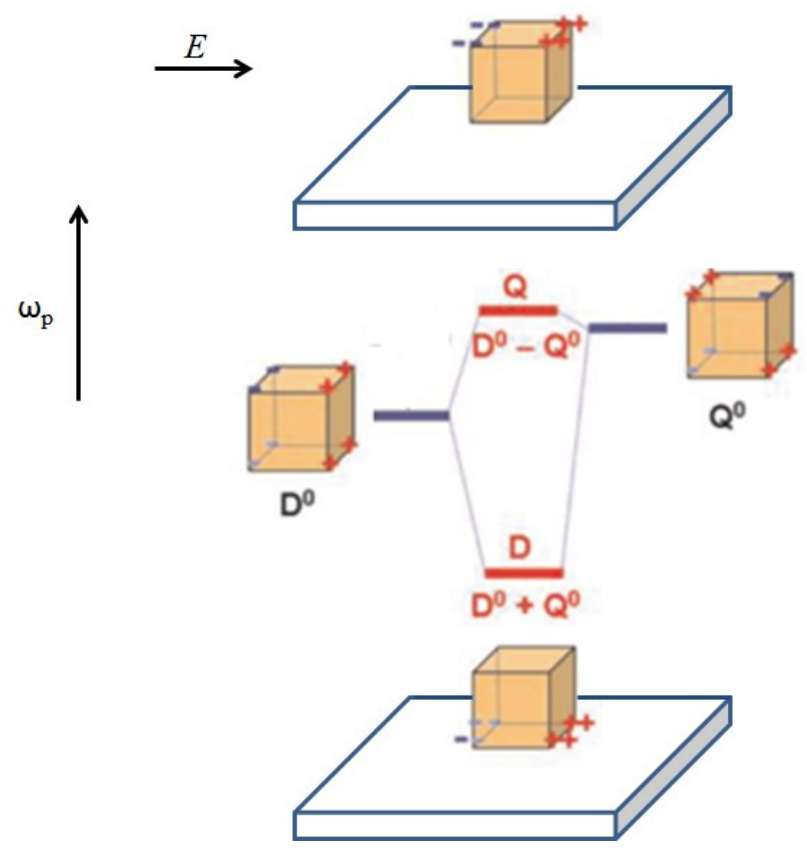

Figure 1.8: Charge distribution of the $D^{0}$ and $Q^{0}$ modes and the charge distribution of the $D$ and $Q$ modes due to substrate induced hybridization. Adapted with permission from $\left({ }^{97}\right)$. Copyright $($ 2011, American Chemical Society.

The refractive index (n) describes how light propagates through a medium, and is described by:

Equation 12

$$
n=\frac{c}{v}
$$

where $\mathrm{c}$ is the speed of light in a vacuum and $\mathrm{v}$ is the phase velocity in the material.

The distance and contact area between the substrate and the particles, and the effective refractive index of the substrate and surroundings all affect the separation between the hybrid D and Q modes. ${ }^{107}$ As such, the LSPR of core-shell nanostructures is dependent on the size and shape of both the core and the shell, as well as the porosity of 
the shell and spacing between the core and shell. ${ }^{90}$ As the refractive index of the substrate and superstrate become more similar, hybridization is lost.

It can be noted that, as the refractive index of the substrate increases, the D mode, which is localized into the substrate, is more redshifted, while the Q mode is unchanged (Figure 1.9, left). On the contrary, when the refractive index of the surroundings is changed (by immersing the substrate in different refractive index solutions), a greater sensitivity is observed by the Q mode, which is localized away from the substrate (Figure 1.9 , right). ${ }^{107}$
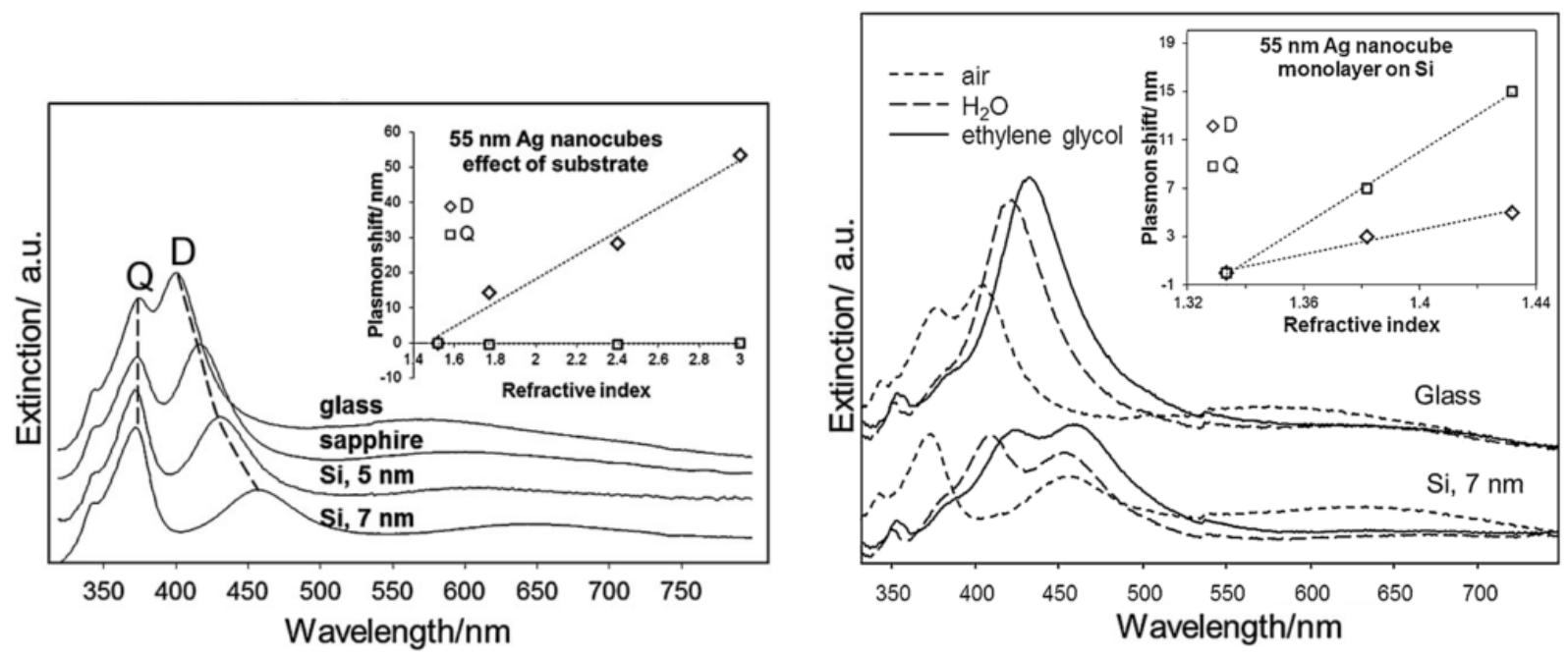

Figure 1.9: UV-Vis spectra of supported silver nanocubes showing sensitivity of $D$ and $Q$ hybrid modes to different refractive indices of substrate (left) and surroundings (right). Reprinted with permission from $\left({ }^{107}\right)$. Copyright $($ C 2012, Springer.

\section{5 - The Langmuir-Blodgett Method}

The Langmuir-Blodgett method is a bottom-up technique that was originally used to assemble amphiphilic molecules, but has since been used for the assembly of a variety of nanomaterials, including many types of nanoparticles and nanorods. ${ }^{108}$ In this method, the particles are suspended on the surface of water. The spacing of the particles is controllable through the use of spacer molecules and by the use of a movable barrier, which can compress and expand the monolayer. At the desired surface pressure, the 
monolayer can be transferred to substrates by vertically dipping the substrate (Blodgett deposition) or by horizontally touching the substrate to the surface and lifting (Schaefer deposition).

\subsection{1 - Langmuir Monolayer Formation}

The nanoparticles are first suspended in a volatile solvent, often chloroform, before being deposited onto the trough. The solvent must be selected such that it can suspend a substantial amount of the desired material, it will evaporate quickly after deposition onto the surface, and it must also not be very soluble with water or some materials may also be drawn into the subphase and precipitate out. ${ }^{109}$ Often lipids, such as DPPE and DOPC, will also be added to the nanoparticle solution before depositing it onto the Langmuir-Blodgett trough, to act as spacers by fitting between the nanoparticles. ${ }^{110}$ This allows the spacing between particles to be controlled by both the use of spacer molecules and by a movable barrier, which can compress and expand the monolayer.

In the formation of the Langmuir monolayer, the nanoparticles are deposited dropwise onto the surface of water, using a syringe held just above the surface. The nanoparticles are able to be suspended on the surface; this is in part due to being stabilized with capping agents, often organic molecules, which make the nanoparticles hydrophobic enough to be suspended on the surface. In the case of the silver nanocubes, poly(vinyl pyrrolidone) attaches to the surface via the nitrogen and oxygen atoms, ${ }^{60}$ allowing them to be suspended on the surface due to its long carbon chain. 


\subsection{2 - Measurement of Surface Pressure}

The surface pressure of the monolayer is determined with the use of a Wilhelmy plate, which is immersed through the air-water interface. ${ }^{109,110}$ The vertical force experienced by the Wilhelmy plate can be expressed as a sum of gravity and surface tension, acting downwards, and buoyancy, acting upwards. The downwards force on the plate is expressed as the following, as a sum of the weight of the plate (term 1), the surface tension (term 2) subtracted by the upwards force due to water displaced by the plate (term 3): ${ }^{109}$

\section{Equation 13}

$$
F=\left(\rho_{P} l w t\right) g+2(w+t)(S T) \cos \theta-\left(\rho_{L} d w t\right) g
$$

where $1, w$, and t are the length, width and height of the plate, respectively; $\rho_{\mathrm{L}}$ and $\rho_{\mathrm{P}}$ are the density of the liquid and the plate, respectively; ST is the surface tension; $\mathrm{g}$ is the acceleration due to gravity; and $\theta$ is the contact angle of the plate and liquid.

The pressure reading will be zeroed before taking any measurements, thus eliminating the weight (term 1) from equation 13. Similarly, the plate is always maintained at a constant level by the balance, even with changes in surface tension, thus eliminating the upthrust (term 3) from the equation. Therefore, the downwards force can then be expressed as: ${ }^{109}$

$$
F=2(w+t)(S T) \cos \theta
$$


This expression can be reduced further, since the plate will contact the liquid at an angle of $\theta=0^{\circ}$. Rearranging the expression to be expressed in terms of surface tension results in: ${ }^{109}$

\section{Equation 15}

$$
S T=\frac{F}{2(w+t)}
$$

The force is measured in $\mathrm{mN}$, and the perimeter, $2(\mathrm{w}+\mathrm{t})$, is measured in meters, which yields the $\mathrm{ST}$ in $\mathrm{mN} / \mathrm{m}$. Surface pressure $\left(\mathrm{P}_{\text {Surf }}\right)$ is defined such that $\mathrm{ST}+\mathrm{P}_{\text {Suf }}$ $=$ constant, where the constant is the absolute surface tension of the liquid $(-72.8 \mathrm{mN} / \mathrm{m}$ for water at 298K, 1 atmosphere). This means that as the surface tension decreases (from adding or compressing a monolayer) the surface pressure will increase. ${ }^{109}$

\subsection{3 - Isotherms and Transferring to Substrates}

As the monolayer is compressed by the movable barrier, a pressure-area isotherm is obtained, which provides information about the particles in the monolayer. Specifically, this isotherm can be divided into distinct phase analogues, representing the degree to which the particles are interacting. ${ }^{109}$ When the monolayer is sufficiently expanded, such that the isotherm's slope is zero, the particles are said to be in 'the gas phase'. In this phase, the particles are spaced such that they are not interacting with each other. As the monolayer continues to be compressed, the particles will enter 'the liquid phase', which is characterized by a slight positive slope in the isotherm. In this phase, the particles are close enough to be interacting with each other, but are still mobile. In some cases, a plateau in the isotherm will divide two liquid phases (liquid-expanded and liquidcondensed), which is a result of particle reorganization. With the continued compression of the monolayer, it will transition into 'the solid phase', which is recognized on the 
isotherm by a sharp, near vertical slope. In this phase, the particles are tightly packed, and have restricted mobility. The isotherm can be used to assist the selection of surface pressure for deposition of the particles onto substrates.

At the desired surface pressure, the monolayer can be transferred to the substrate by one of two methods: Blodgett deposition, which involves vertically dipping the substrates, or Schaefer deposition, which involves horizontally lifting the substrates (Figure 1.10). ${ }^{111}$ With a hydrophilic substrate, Blodgett deposition can be employed by immersing the substrate across the air-water interface while the monolayer is at a low pressure. The monolayer can then be compressed to the desired surface pressure, and the substrate can be slowly removed from the meniscus. For a hydrophobic substrate, the deposition would occur while lowering the substrate, depositing into the meniscus. Contrarily, Schaefer deposition is appropriate for hydrophobic substrates.
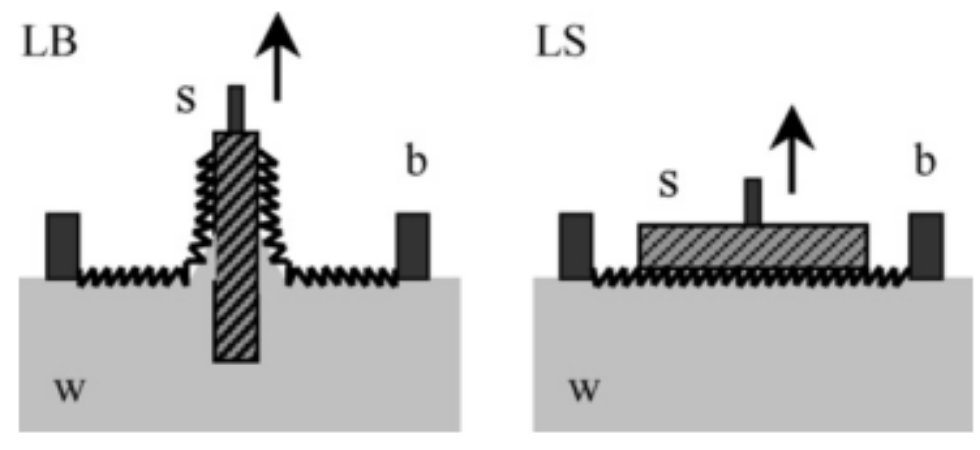

Figure 1.10: Blodgett deposition (left) and Schaefer deposition (right). Reprinted with permission from $\left({ }^{111}\right)$. Copyright $\odot 2006$, Elsevier.

\section{6 - Polymer Thin Films as Substrates for Silver Nanocubes}

In this work, polymer thin films were prepared and used as substrates for silver nanocubes. After the nanocubes were deposited on these polymer films, they were heated above the polymer's glass transition temperature to allow the embedding of the nanocubes into the films to a controlled depth. This allows for the growth of partial 
copper oxide shells on the exposed portions of the silver nanocubes. Properties of polymer thin films, specifically the glass transition temperature, and embedding of nanoparticles into polymer films are the topics of the next sections.

\subsection{1 - Properties of Polymer Thin Films: Glass Transition Temperature}

Polymers are large molecules made up of many repeating subunits and can be roughly categorized as crystalline (highly ordered molecular structures) or amorphous; however, most polymers tend to have characteristics of both. Unlike crystalline polymers, which have a distinct melting temperature, some amorphous polymers have a glass transition temperature $\left(\mathrm{T}_{\mathrm{g}}\right)$, above which the polymer will behave rubbery. ${ }^{112}$ In particular, the $T_{g}$ can be defined as the kinetic arrest upon cooling a fluid. ${ }^{113}$ This transition occurs over a range of temperatures with the $T_{g}$ being defined as the point at the intersection of the two lines (Figure 1.11).

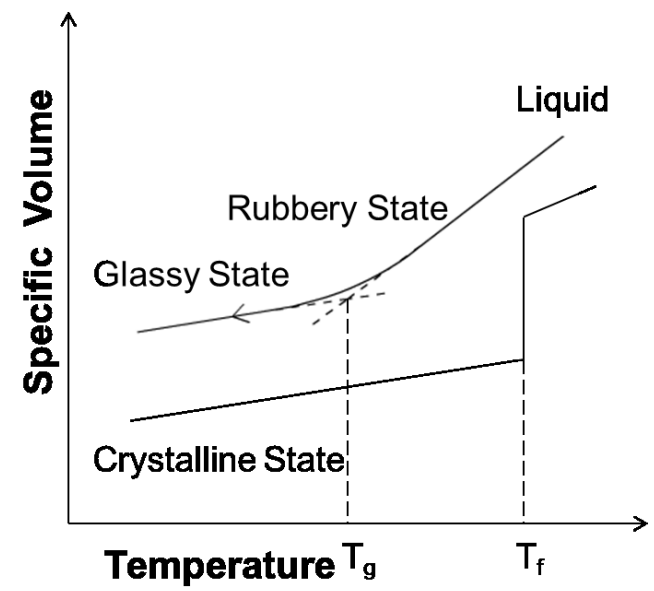

Figure 1.11: Specific volume vs temperature, indicating the glass transition temperature $\left(T_{g}\right)$, glassy and rubbery state for amorphous polymers and the crystalline state and freezing temperature $\left(T_{f}\right)$ for crystalline polymers.

At the $\mathrm{T}_{\mathrm{g}}$ the polymer chains are more able to flow or move around one another. As such, the $T_{g}$ is greatly affected by anything that influences the motion of the chains, with factors hindering the free rotation of the polymer chains increasing the $\mathrm{T}_{\mathrm{g}}{ }^{12}$ This 
can include the stiffness of the polymer chains, cross-linking and intermolecular forces between chains, and side groups on the backbone. Particularly, groups that increase the rigidity of the chains, including groups that result in partial double bond character on the backbone, hinder the rotation around said bonds, increases the $\mathrm{T}_{\mathrm{g}}$. Similarly, both crosslinking and intermolecular forces restrict the movement of the polymer chains around one another; therefore, as the degree of cross-linking and intermolecular forces increases, so does the $T_{g}$. Furthermore, bulky groups (including the phenyl group on polystyrene) also restrict movement of the chains, again resulting in an increase in the $\mathrm{T}_{\mathrm{g} .}{ }^{112}$

In addition to properties of the polymers themselves, existence of the polymer as a thin film can also alter the $\mathrm{T}_{\mathrm{g}}$. In particular, the effects of both the substrate-polymer and the polymer-air interfaces have to be considered. ${ }^{112,113}$ Often, a model that assumes the existence of different layers in the film is employed. ${ }^{114,115}$ The top layer (at the polymerair interface) is the mobile surface layer, a 'liquid-like' layer, which has different properties from the bulk due to the lack of bordering polymer. This can result in lower mass densities and less restriction for polymer rearrangements. ${ }^{116}$ The intermediate layer has bulk-like properties; this bulk-like layer decreases in thickness as the film becomes thinner. ${ }^{114}$ The bottom layer (at the polymer-substrate interface) generally has an increased density, which increases with increasing attractive interaction between the polymer and substrate. ${ }^{114}$ As the density of the thin film increases, the $T_{g}$ will also increase. ${ }^{113}$ As the film becomes thinner, the average density generally increases, and a greater proportion of the film is made up by the mobile surface layer; this can result in an increase or decrease of the $\mathrm{T}_{\mathrm{g}}$ depending on the relative contributions. ${ }^{114,116}$ 


\subsection{2 - Nanoparticle Embedding into Polymer Thin Films}

In this work, the nanoparticles are deposited onto polystyrene thin films, which are subsequently heated above polystyrene's $T_{g}$. This increases the mobility of the polymer chains, allowing the initial formation of a void in its surface, enabling the nanoparticles to become incorporated. However, certain conditions must be met in order for the nanocubes to begin to embed into the polymer thin film, with the thermodynamic

driving force of embedding being the decrease in Helmholtz free energy $\left(\mathrm{A}_{\mathrm{S}}\right) .{ }^{117}$ The Helmholtz free energy change can be described for a system where $a$ is the interfacial area, $\gamma_{1}$ is the surface energy of a non-interacting nanoparticle surface and $\gamma_{12}$ is the surface energy between the nanoparticle and the substrate; this gives initial state $A_{S}=a \gamma_{1}$ and final state $A_{S}=a \gamma_{12}$. Therefore, the change in surface free energy can be described as: ${ }^{117}$

\section{Equation 16}

$$
\Delta A_{S}=a\left(\gamma_{12}-\gamma_{1}\right)
$$

These conditions $\left(\gamma_{1}>\gamma_{12}\right.$, such that $\left.\Delta \mathrm{A}_{\mathrm{S}}<0\right)$ must be satisfied for the nanoparticles to begin to embed into the polymer. However, this will only indicate if the nanoparticles will start to embed, and not the extent to which they will be incorporated. In order for this sinking process to continue to completion, it is necessary that the surface energy of the particle $\left(\gamma_{1}\right)$ is greater than the sum of the interfacial energy $\left(\gamma_{12}\right)$ and the surface energy associated with the polymer reformation $\left(\gamma_{2}\right)$, that is: ${ }^{117}$

$$
\gamma_{1}>\gamma_{2}+\gamma_{12}
$$


However, the system may end up in an intermediate regime if the surface energy of the underlying polymer is too high to allow complete incorporation (polymer reforming around the particle). This can be observed experimentally as incomplete incorporation of the particles, and is expressed as: ${ }^{117}$

\section{Equation 18}

$$
\gamma_{12}<\gamma_{1}<\gamma_{2}+\gamma_{12}
$$

In addition, it is important to note that the nanoparticles used in this work were passivated with PVP, hence the surface energy of the particle is the surface energy of the capping agent. ${ }^{117}$ The surface energy of bulk $\mathrm{PVP}^{117}$ is $\sim 43 \mathrm{~mJ} \mathrm{~m}^{-2}$ and bulk polystyrene ${ }^{118}$ is $33 \mathrm{~mJ} \mathrm{~m}^{-2}$. As such, since the surface energy of PVP is greater than that of polystyrene, the particles can begin to embed.

Consequently, for the system of interest presently, the surface energy of the capping agent $\left(\gamma_{\text {cap }}\right)$, PVP, should be considered instead of the surface energy of the particle. Similarly, the surface energy of polystyrene $\left(\gamma_{\mathrm{ps}}\right)$ (the substrate) and the surface energy of the interface between the PVP capping agent and the polystyrene ( $\left.\gamma_{\text {cap,ps }}\right)$ should be considered. This allows the Helmholtz free energy change to be defined for this system as:

Equation 19

$$
\Delta A_{S}=a\left(\gamma_{c a p, p s}-\gamma_{c a p}\right)
$$

Additionally, the experimentally observed regime, in which the particles do not fully embed, can be described as:

$$
\gamma_{c a p, p s}<\gamma_{c a p}<\gamma_{p s}+\gamma_{c a p, p s}
$$




\subsection{3 - Spectroscopic Measurement of Silver Nanocube Embedding}

When silver nanocubes are deposited on a substrate, spatially separated hybrid dipolar and quadrupolar modes are produced, ${ }^{97}$ which can allow the nanocubes to act as a 'nanoruler'. This further allows the embedment of the nanocubes into the polymer to be monitored spectroscopically. The modes have different sensitivities during the embedding, and the spectroscopic changes can be divided into four stages, with particular changes to the D, Q and M modes (Figure 1.12). Specifically: ${ }^{117}$

1. Redshift of D mode, no change in $\mathrm{Q}$ mode, new M mode emerges. This stage occurs as the nanocubes just begin to embed, and D mode is the most sensitive in this stage.

2. Both the $\mathrm{D}$ and $\mathrm{Q}$ modes redshift, $\mathrm{M}$ mode has a two state-like transition. In this stage, the nanocubes begin to tilt.

3. D mode reaches maximum, $\mathrm{Q}$ mode has greatest redshift, and only second $\mathrm{M}$ mode remains. In this stage, the nanocubes have embedded about half-way, a lot of tilting is observed and the Q mode is most sensitive in this stage.

4. Both the $\mathrm{D}$ and $\mathrm{M}$ mode remain constant, $\mathrm{Q}$ continues to redshift and begins to become obstructed as it overlaps with the D mode. In this stage, the majority of the nanocube is embedded, often with just a single corner exposed. This stage has the least sensitivity and loss of anisotropy of the environment.

Specifically, as the nanocubes begin to embed, the hybrid dipolar mode, which is more sensitive to the polystyrene substrate, will redshift first, due to the effective increase in refractive index. As the nanoparticles continue to embed and reach approximately the 
half-way point, the hybrid quadrupolar mode, which is sensitive to the superstrate, will begin to experience a change in refractive index, resulting in a shift in this mode. ${ }^{117}$

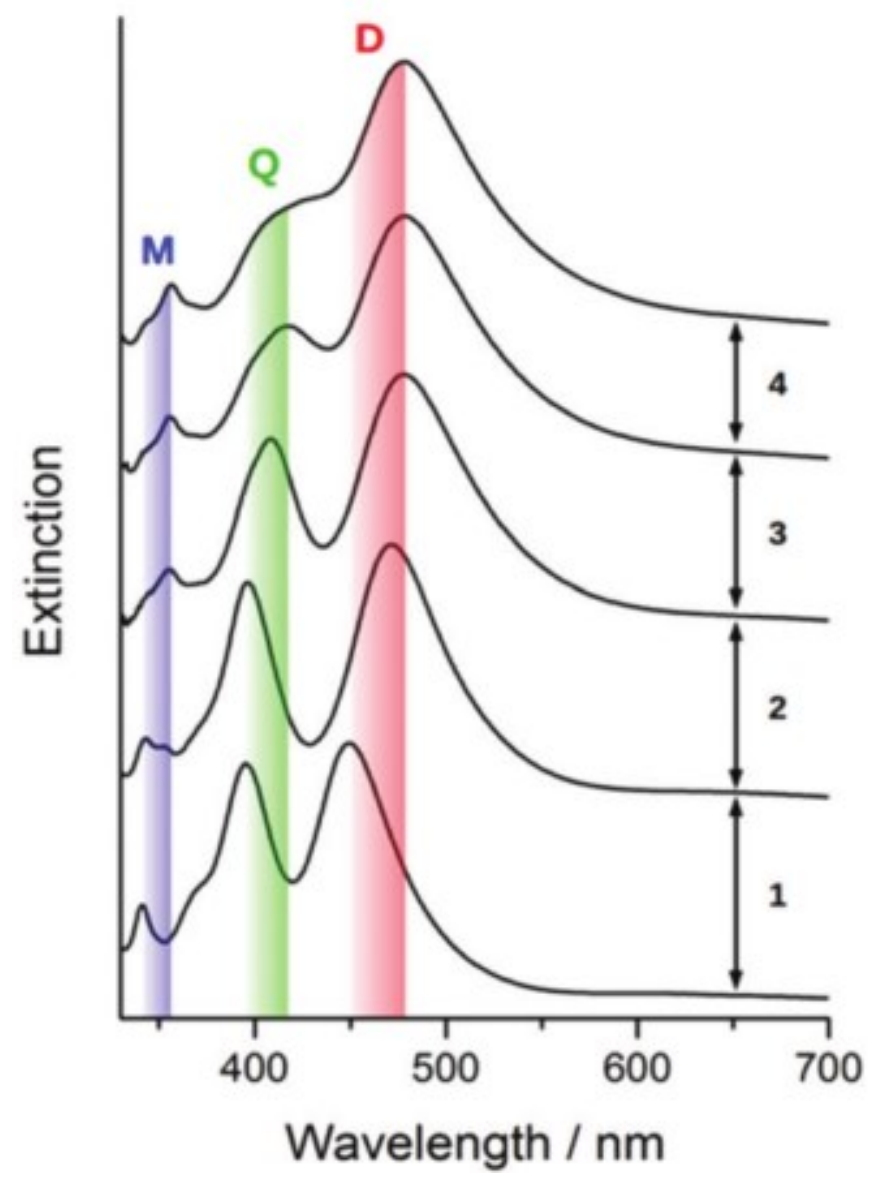

Figure 1.12: Plasmonic modes monitored with UV-Vis spectroscopy as silver nanocube monolayers are embedded into polystyrene thin films. Reprinted with permission from $\left({ }^{117}\right)$. Copyright $(2) 2016$, Royal Society of Chemistry.

\section{7 - Experimental Set-ups for Copper Oxide Growth}

In the current approach to the preparation of silver nanocubes with partial copper oxide shells (overview described in Section 1.1.4), it was necessary that an experimental set-up be designed such that the growth could be controlled and reproducible. In particular, it is necessary that reagents are able to mix thoroughly in solution, such that they are able to diffuse to the surface of the particle. In order to address this issue, set-ups were created whereby the slide was attached to a portion which spins, thus stirring the 
solution and ensuring that the solution is adequately mixed. Since the slide is attached to a moving piece, it is necessary that the set-up allows for steady attachment of the substrate, such that it would not fall off during the course of the reaction. Moreover, it was desired that the stirring would not agitate the solution considerably, as to cause bubbles, which could interfere with the reactions. As such, throughout the course of this work, four set-ups were investigated, namely the 'Mechanical Stirrer' (M), 'Puck' (P), 'Teflon piece' (T), and the 'New Set-up' $(\mathrm{N})$, which are described in the following sections.

\subsection{1 - Mechanical Stirrer (M)}

The original set-up used for the growth of copper oxide shells on embedded silver nanocubes involved a steel rod onto which a glass slide had been epoxied, so that the sample could be taped to it (Figure 1.13). The rod was fit into a laboratory stirrer, which spun the rod and sample (Figure 1.13). In this set up, as the sample was taped onto the slide attached to the rod, the sample was inverted in the solution (Figure 1.13).

This sample was initially prepared for ease and convenience, and as an initial test as a set-up for the production of copper oxide shells. However, the use of a steel rod onto which a slide had been epoxied presented the possibility of introduction of contaminants and a challenge for cleaning. In addition, only one speed was possible for the set-up, and the ability to adjust the speed of stirring is desirable. As such, in an attempt to overcome these issues, the next set-up, the 'puck' was investigated. 


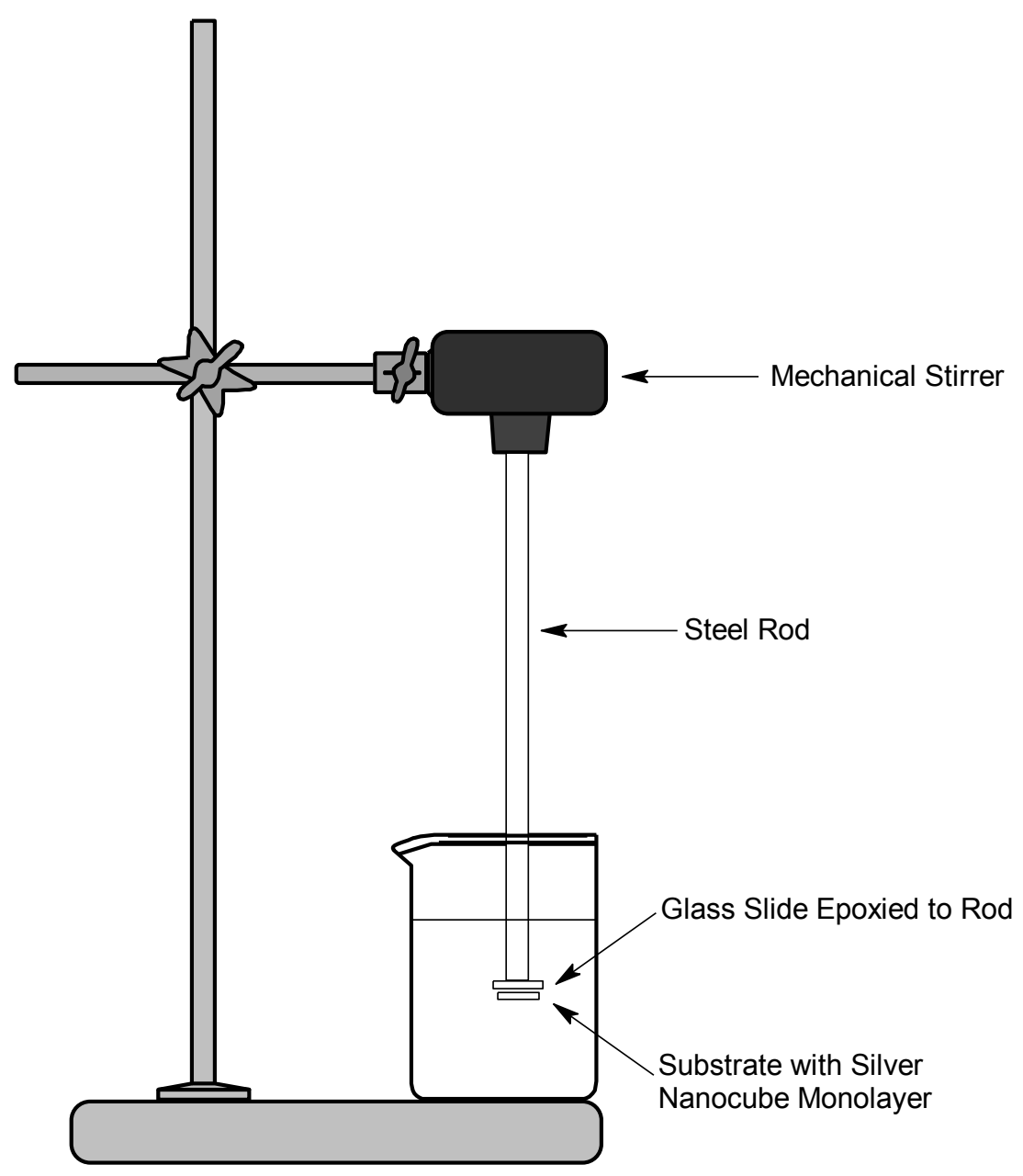

Figure 1.13: Schematic of 'Mechanical Stirrer'. Diagram created with ACD/ChemSketch, version 14.01 Advanced Chemistry Development, Inc., Toronto, On, Canada, www.acdlabs.com, 2015.

\subsection{2 - Puck (P)}

Subsequently, another set-up was explored for growth of partial copper oxide shells, where the sample was taped onto the top surface of a small puck, which contained a magnet (Figure 1.14). The reaction would be carried out on a stirring hot-plate, thus allowing the puck to spin, spinning the sample and stirring the solution (Figure 1.14).

In this set-up, the speed of spinning is adjustable by altering the stirring speed on the hotplate. In addition, as the substrate is affixed to the top of the puck, this set-up is sturdier, in the sense that the slide is less likely to fall off. However, by nature of the design of this set-up, the stirring rate was slower than other set-ups. In addition, the puck 
must be centered in the beaker or it will shake back and forth, whereby it is no longer smoothly stirring the solution. As an attempt to further improve upon this set-up, the 'Teflon piece' was developed.

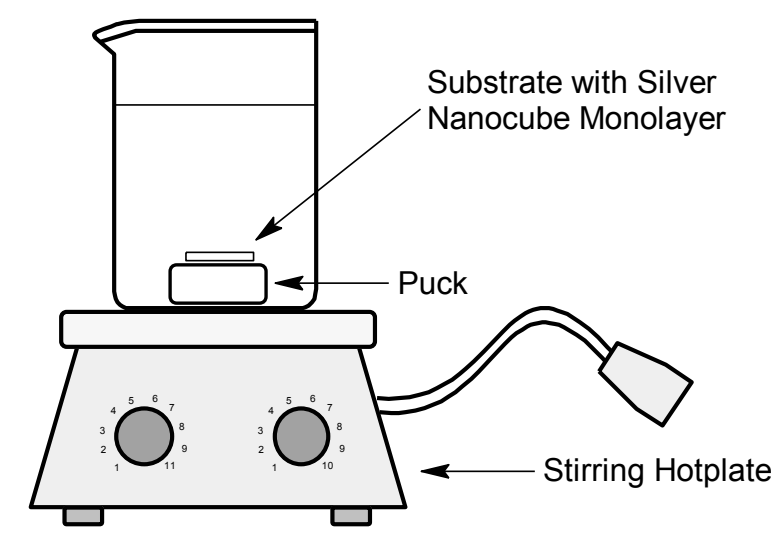

Figure 1.14: Schematic of 'Puck'. Diagram created with ACD/ChemSketch, version 14.01 Advanced Chemistry Development, Inc., Toronto, On, Canada, www.acdlabs.com, 2015.

\subsection{3 - Teflon Piece (T)}

The 'Teflon Piece' set-up was prepared with a cylinder of Teflon, to which the substrate with the silver nanocube monolayer is adhered, attached to a rotary tool (Figure 1.15). An adjustable stand was used to support the beaker (Figure 1.15), allowing it to be raised and lowered, for ease of removal of the sample.

In this set-up, the speed could be adjusted by changing the voltage on the Variac. In addition, the Teflon piece was removable, making cleaning easy, reducing risk of introducing contaminants run-to-run. However, the production of bubbles was an issue with this set-up, especially since oxygen can react with hydrazine. ${ }^{119}$ In addition, this reaction is $\mathrm{pH}$ dependent and $\mathrm{Cu}^{2+}$ has been reported to catalyze this reaction, ${ }^{119}$ further increasing the issue. As such, it was desired to construct a set-up which overcomes this issue, leading to the development of the 'New Set-up'. 


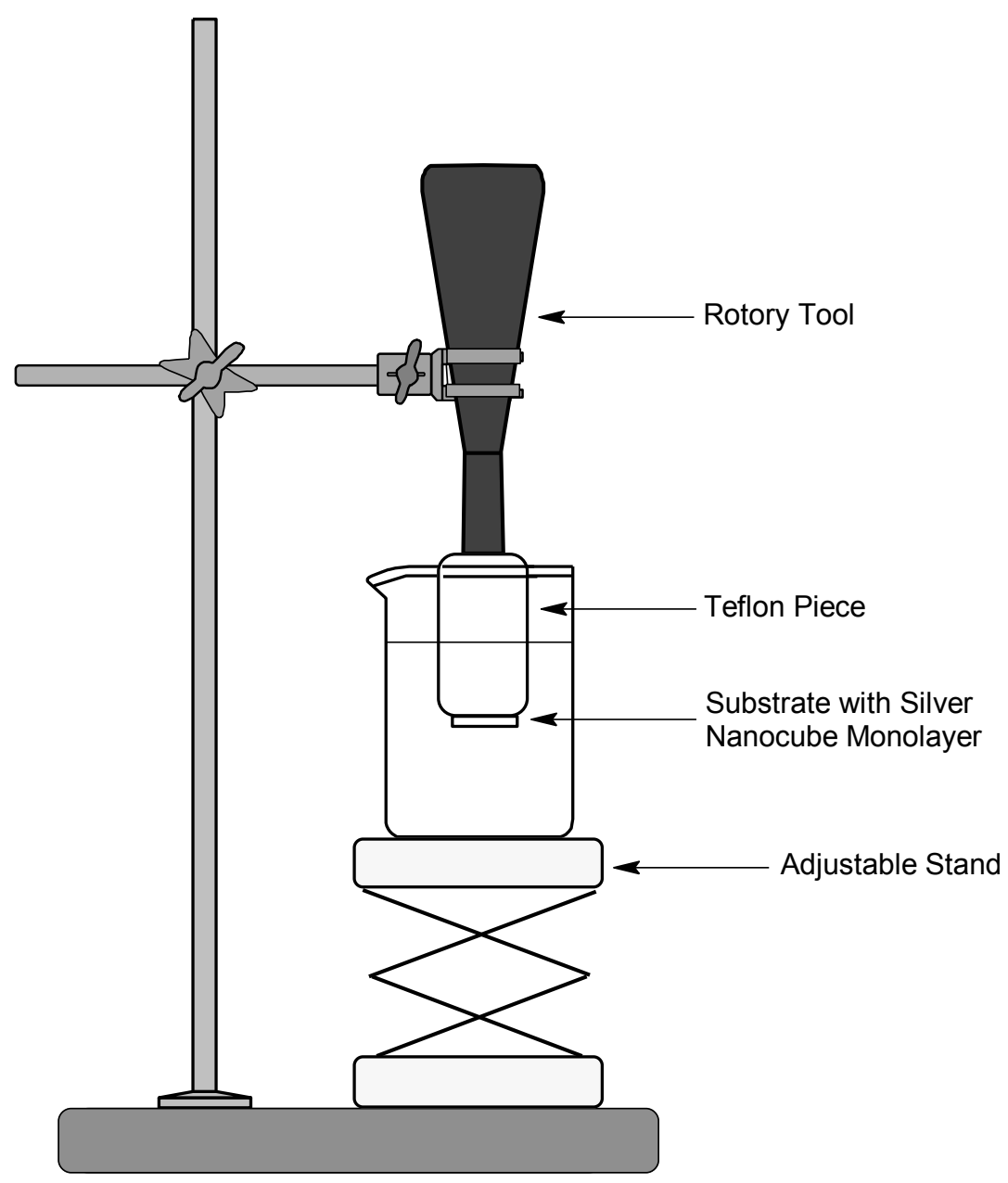

Figure 1.15: Schematic of 'Teflon Piece'. Diagram created with ACD/ChemSketch, version 14.01 Advanced Chemistry Development, Inc., Toronto, On, Canada, www.acdlabs.com, 2015.

\subsection{4 - New Set-up (N)}

In order to improve upon the previous set-ups, the newest set-up was produced, whereby the reaction is carried out in a three-necked flask. A glass rod is attached to a mechanical stirrer, and the sample is affixed to the glass rod, inserted through the middle opening (Figure 1.16). The three ports allows nitrogen to be continuously flowing through the reaction through one opening, with the stirring rod through the middle opening, and reagents added through the final opening (Figure 1.16).

This set-up allows the reaction to be carried out under a constant flow of nitrogen, which eliminates the issue of a side reaction of hydrazine with oxygen. Additionally, the 
stirrer can be set to various speeds. As well, the glass rod is removable, and the set-up overall is easy to clean.

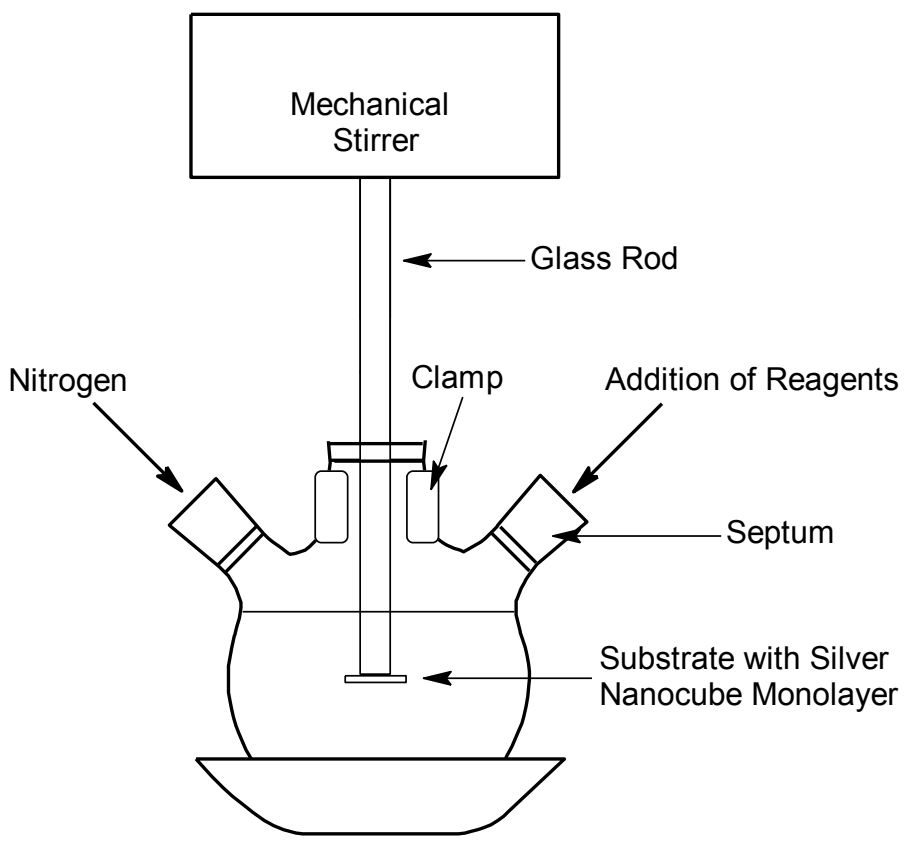

Figure 1.16: Schematic of the 'New Set-up'. Diagram created with ACD/ChemSketch, version 14.01 Advanced Chemistry Development, Inc., Toronto, On, Canada, www.acdlabs.com, 2015.

\section{8 - Concluding Statements}

The goal of the present work is to produce anisotropic core-partial shell silvercopper oxide nanoparticles. It was hypothesized that through the use of a sacrificial template, namely the polystyrene thin films into which the nanocubes were embedded, these types of core-partial shell particles could be produced. In addition, several set ups were considered for the productions of said particles, each of which was based on the goal of controllably producing core-partial shell nanoparticles. In these set-ups, the stirring rate, orientation and stability varies between, and is expected to have an effect on the resultant morphologies. Furthermore, in this system the order of addition of reagents is also varied, which is expected to have an effect on the kinetics of the reaction, and thus the produced morphologies as well. In addition, since the shell will not be fully engulfing 
the nanoparticle, it is expected that these core-partial shell nanoparticles will have hybridization of the plasmonic modes while the particles are suspended in solution.

In this introduction, the necessary concepts to understand the current system were elucidated, namely, anisotropy, both morphological and chemical, and the properties, applications and synthesis of said types of particles. The method of production of the particles in this work was described, namely, partially embedding silver nanocubes into polystyrene thin films and growing copper oxide on the exposed surface of the cubes.

The synthesis of nanoparticles was described, with specific focus on the Polyol process as a method for the production of silver nanocubes, the core particle of choice for this work. Nanoparticle nucleation and growth, as well as factors that control the morphology of the resulting particles, were discussed. Copper oxide, the material grown on the surface of the silver nanocubes, was described, in terms of its structure, properties and potential applications, as well as its synthesis.

Silver is a plasmonic material, and characterization of the embedding process and growth of copper oxide makes use of this property. The use of the Langmuir-Blodgett method as a way to deposit the silver nanocubes onto the polystyrene substrate in a controlled manner was explored, with the necessity of keeping the particles adequately spaced so that the shells can be grown considered. The glass transition temperature and embedding of the silver nanocubes into polystyrene thin films, and monitoring the process by observing the changes to the hybrid D and hybrid Q modes, was described. Hence, in this introduction, the necessary theory involved with the production of these core-partial shell nanoparticles was described. 


\section{2 - Methods: Copper Oxide Coating on Silver Nanocubes}

\section{1 - Substrate Preparation}

\subsection{1 - Synthesis of Silver Nanocubes}

For this work silver nanocubes were synthesized by the Polyol Process. ${ }^{56}$ All glassware is first soaked in Aqua Regia (1 part nitric acid (Anachemia), 3 parts hydrochloric acid (Anachemia)), and then rinsed 52 times in tap water, 32 times in distilled water and 16 times in deionized water (Milli-Q, 18.2 M $\cdot \mathrm{cm}$ ). To begin the synthesis, $35 \mathrm{~mL}$ of ethylene glycol (EG, 99.8\%, Sigma-Aldrich) was heated with $0.4 \mathrm{~g}$ of poly(vinylpyrrolidone) (PVP, MW 55000 , Sigma-Aldrich) to $150^{\circ} \mathrm{C}$. After this mixture had been heated for a minimum of $1 \mathrm{~h}, 25 \mu \mathrm{L}$ of $0.48 \mathrm{M} \mathrm{NaCl}$ (Sigma-Aldrich) in EG was added to the reaction, followed by $175 \mu \mathrm{L}$ of $0.003 \mathrm{M} \mathrm{Na}_{2} \mathrm{~S}$ (Sigma-Aldrich) in $\mathrm{EG}$, and finally $1.5 \mathrm{~mL}$ of $0.28 \mathrm{M} \mathrm{AgNO}_{3}(99+\%$, Sigma-Aldrich) in EG was added at an injection rate of $0.5 \mathrm{~mL} / \mathrm{min}$. UV-Vis spectroscopy was used to monitor the reaction, and upon the appearance of a peak at $350 \mathrm{~nm}$ a second injection of $1.5 \mathrm{~mL}$ of $0.28 \mathrm{M} \mathrm{AgNO}_{3}$ in EG was added at a rate of $1 \mathrm{~mL} / \mathrm{min}$. The reaction was quenched in an ice bath when the spectrum had a dipole peak at $480 \mathrm{~nm}$ and a sharp peak at $350 \mathrm{~nm}$ (multipolar, signifying the cubic shape). The nanoparticles were then centrifuged (Thermo Legend Micro21) at $12000 \mathrm{~g}$ 's for 30min and re-suspended in ethanol. The particles were further cleaned by centrifuging (12000 g's, 10min) and re-suspending in fresh ethanol (Commercial Alcohols, 95\%) a minimum of 4 times. 


\subsection{2 - Preparation of Polystyrene Thin Films}

Polystyrene thin films were prepared by spin-coating. First, a solution of $3 \mathrm{wt} \%$ polystyrene (Sigma Aldrich, 192,000 g/mol) in toluene (Anachemia) was prepared and stirred at $300 \mathrm{rpm}$, for a minimum of $3 \mathrm{~h}$ before filtering the solution through a $0.22 \mu \mathrm{m}$ syringe filter.

Glass slides were cleaned by sonicating in methanol (BDH, VWR Analytical, 99.8\%) for $5 \mathrm{~min}$, rinsed in methanol, rinsed in deionized water and dried in the oven. Using a homemade spincoater, the polystyrene solution was added to the surface of the slide, such that it completely covered the surface, and the spincoater was then turned on to $\sim 4000 \mathrm{rpm}$ for a time of $1 \mathrm{~min}$. The slides were then left at room temperature for a minimum of $1.5 \mathrm{~h}$ to allow the solvent to evaporate before transferring to the oven $(\geq 110$ ${ }^{\circ} \mathrm{C}$ ) and leaving overnight, to anneal the films.

\subsection{3 - Langmuir Blodgett Deposition of Silver Nanocubes}

The Langmuir Blodgett method was used to deposit silver nanocubes on polystyrene thin films. The Langmuir Blodgett trough was cleaned with chloroform (Alfa Aesar, 99.5\%) using surfactant free delicate task wipers (KIM TECH), and was then filled with deionized water (Milli-Q, 18.2 $\mathrm{M} \Omega^{*} \mathrm{~cm}$ ). The water was aspirated off, and was then refilled and let sit for $20 \mathrm{~min}$. A pressure-area isotherm was taken and used to determine the cleanliness of the trough. If the pressure measured was greater than 0.2 $\mathrm{mN} / \mathrm{m}$ the surface was aspirated off and refilled with deionized water, and then let sit for 20 min before taking an isotherm. This process was repeated until the pressure was less than or equal to $0.2 \mathrm{mN} / \mathrm{m}$. 
The silver nanocube samples were prepared by centrifuging at 12000 g's for 10min, and redistributing in chloroform. The sample was then centrifuged at $12000 \mathrm{~g}$ 's for $5 \mathrm{~min}$ and the supernatant was replaced with fresh chloroform, twice. The sample was then dried under $\mathrm{N}_{2}$ gas, and then $200 \mu \mathrm{L}$ of chloroform and $10 \mu \mathrm{L}$ of DOPC lipid (Avanti Polar Lipids) was added, and sonicated. This mixture was added to the trough dropwise with a microsyringe (Hamilton, $500 \mu \mathrm{L}$ ). After the monolayer was let sit for 20 min a pressure-area isotherm was obtained at a speed of $35 \mathrm{~cm}^{2} / \mathrm{min}$ and the trough was opened by taking a negative isotherm at the same speed. Once at the desired pressure the barrier was set to 'pressure control', and the monolayer was transferred with the use of Schaefer deposition (horizontally touching the slide to the surface and lifting). The slides were dried with $\mathrm{N}_{2}$ and stored in a desiccator until use.

\subsection{4 - Embedding Silver Nanocubes into Polystyrene Thin Films}

The substrates were partially embedded into the polystyrene thin films by heating above polystyrene's glass transition temperature. Specifically, the hot plate was set to a temperature of $\sim 115^{\circ} \mathrm{C}$, measured with a thermocouple. The slide would be placed on for 10s and then measured with UV-Vis spectroscopy. This process was continued until the dipolar mode had stopped red-shifting and the quadrupolar mode had just begun to shift $(\sim 15 \mathrm{~nm})$.

\section{2 - Set-ups for Growth of Copper Oxide Shells}

Four set-ups were used for the growth of copper oxide shells on the surface of silver nanoparticles, namely, the 'Mechanical Stirrer' (described in section 1.7.1), the 'Puck' (described in section 1.7.2), the 'Teflon Piece' (described in section 1.7.3) and the 'New Set-up' (described in section 1.7.4). 


\subsection{1 - Mechanical Stirrer: Set-up Parameters}

The sample was taped onto a glass slide affixed to the steel rod. This rod was then

inserted into the Laboratory Stirrer. With this set up trials were conducted by slowly adding hydrazine (Section 2.3.1) or slowly adding copper (Section 2.3.2) with and without lactic acid.

\subsection{2 - Puck: Set-up Parameters}

The sample was taped to a custom puck, containing a magnet. This was then placed in a beaker on a stirring hot plate (CORNING PC-402D) and set to $1000 \mathrm{rpm}$. With this set up trials were conducted by slowly adding hydrazine (Section 2.3.1) or slowly adding copper (Section 2.3.2) with and without lactic acid.

\subsection{3 - Teflon Piece: Set-up Parameters}

The sample was taped onto the custom Teflon cylinder, which was inserted into a rotary tool (dremel model 800 ). The ACDC converter was set to $6 \mathrm{~V}$ and the Variac was set to $100 \mathrm{~V}$. With this set up trials were conducted by slowly adding hydrazine (Section 2.3.1) or slowly adding copper (Section 2.3.2) with and without lactic acid.

\subsection{4 - New Set-up: Set-up Parameters}

The sample was taped onto a glass rod, which was inserted into a stirrer (IKA ${ }^{\circledR}$ RW16 basic). The stirrer was set to 10, the highest rate. Prior to beginning the synthesis, the solution was purged with nitrogen for at least $15 \mathrm{~min}$. With this set up the concentrations of $\mathrm{NaOH}$ and $\mathrm{Cu}\left(\mathrm{NO}_{3}\right)_{2}$ were varied, as described in (Section 2.3.3). 


\section{3 - Synthesis Methods of Copper Oxide Shells}

\subsection{1 - Copper Oxide Growth: 'Slow' Addition of Hydrazine}

For samples prepared with additions of hydrazine (Fluka, 25\%) throughout the reaction, the solution was first prepared by adding $250 \mathrm{~mL}$ of deionized water. Note that in the case of the New Set-up, the solution would first be purged with $\mathrm{N}_{2}$ gas for a minimum of $15 \mathrm{~min}$. The substrate would then be attached to the set-up and inserted into the water. Subsequently, $450 \mu \mathrm{L}$ of $5 \mathrm{M} \mathrm{NaOH}$ (BioShop, 98\%), $450 \mu \mathrm{L}$ of $4 \mathrm{M}$ lactic acid (if using), and $450 \mu \mathrm{L}$ of $0.5 \mathrm{M} \mathrm{Cu}\left(\mathrm{NO}_{3}\right)_{2}$ were added. A solution of $220 \mu \mathrm{L}$ of $25 \%$ hydrazine in $10 \mathrm{~mL}$ of deionized water had been prepared, and was added in $1 \mathrm{~mL}$ aliquots every $5 \mathrm{~min}, 10 \mathrm{x}$, for a total reaction time of $50 \mathrm{~min}$.

\subsection{2 - Copper Oxide Growth: 'Slow' Addition of Copper Nitrate}

For samples prepared with additions of copper nitrate throughout the reaction, the solution was prepared first by adding $250 \mathrm{~mL}$ of deionized water. Note that in the case of the New Set-up, the solution would first be purged with $\mathrm{N}_{2}$ gas for a minimum of 15 min. The substrate would then be attached to the set-up and inserted into the water. Subsequently, $450 \mu \mathrm{L}$ of $5 \mathrm{M} \mathrm{NaOH}$, and $220 \mu \mathrm{L}$ of $25 \%$ hydrazine were added. A solution of $450 \mu \mathrm{L}$ of $0.5 \mathrm{M} \mathrm{Cu}\left(\mathrm{NO}_{3}\right)_{2}$ and $450 \mu \mathrm{L}$ of $4 \mathrm{M}$ lactic acid (if using) in $10 \mathrm{~mL}$ of deionized water was prepared, and was added in $1 \mathrm{~mL}$ aliquots every $5 \mathrm{~min}, 10 \mathrm{x}$, for a total reaction time of $50 \mathrm{~min}$.

\subsection{3 - Copper Oxide Growth: $\mathrm{NaOH}$, Lactic Acid and $\mathrm{Cu}\left(\mathrm{NO}_{3}\right)_{2}$ Concentrations}

The solution was first prepared by first adding $250 \mathrm{~mL}$ of deionized water. To this varying amounts of $5 \mathrm{M} \mathrm{NaOH}, 4 \mathrm{M}$ lactic acid and then $0.5 \mathrm{M} \mathrm{Cu}\left(\mathrm{NO}_{3}\right)_{2}$, namely 350 
$\mu \mathrm{L}, 450 \mu \mathrm{L}$ or $550 \mu$ of each were added. Then $220 \mu \mathrm{L}$ of $25 \%$ hydrazine was added and the reaction was then let progress for $50 \mathrm{~min}$, in each case.

An additional trial was done without lactic acid. Again, $250 \mathrm{~mL}$ of deionized water was added. Following, varying amounts of $5 \mathrm{M} \mathrm{NaOH}$, and then $0.5 \mathrm{M} \mathrm{Cu}\left(\mathrm{NO}_{3}\right)_{2}$ were added, namely $350 \mu \mathrm{L}, 450 \mu \mathrm{L}$ or $550 \mu$ of each were added. Then $220 \mu \mathrm{L}$ of $25 \%$ hydrazine was added and the reaction, again, was let progress for $50 \mathrm{~min}$, in each case.

\section{4 - Characterization Methods}

\subsection{1 - Characterization with AFM}

The polystyrene thin-films, monolayers prepared with the Langmuir-Blodgett method, embedded monolayers and selected samples after copper oxide growth were characterized with atomic force microscopy (AFM, Ntegra NT-MDT). Images were obtained in semi-contact mode by a silicon probe with a reflective gold coating (NSG03, NT-MDT). The probes used had a $1.75 \mathrm{~N} / \mathrm{m}$ spring constant.

\subsection{2 - Removal and Characterization with UV-Vis Spectroscopy and TEM}

After growth of copper oxide on the surface of the embedded nanocubes, the particles were removed by dissolving the polystyrene support in toluene. The particles were then sonicated to re-suspend the particles and UV-Vis spectra were obtained.

In addition the samples were drop-casted onto a TEM grid (Electron Microscopy Sciences, CF300-Cu), rinsed with toluene and let dry. The TEM images were acquired on a FEI Tecnai G2 F20 TEM at the Carleton University Nano-imaging Facility. 


\section{5 - Theoretical Calculations}

\subsection{1 - Finite Difference Time Domain Calculations}

Finite difference time domain modeling was performed by Dan Prezgot using Lumerical Solutions Inc. (FDTD Solutions v8.15). A $70 \mathrm{~nm}$ silver nanocube, with corner radius of $14 \mathrm{~nm}$ and a $\mathrm{Cu}_{2} \mathrm{O}$ square pyramid with a $140 \mathrm{~nm}$ base and $70 \mathrm{~nm}$ height were the simulation objects. The materials were described by an analytical model fit to tabulated permittivity data. A mesh override region extending at least $10 \mathrm{~nm}$ past the nanocube was used with a $0.5 \mathrm{~nm}$ mesh size. A total-field scattered-field (TFSF) light source was used, and perfect matched layer (PML) was used for the boundary conditions.

\section{3 - Results and Discussion}

The overall goal of this work was to produce anisotropic core-partial shell silvercopper oxide nanoparticles. As such, in this section the results from the anisotropic growth of copper oxide on supported silver nanocubes with the use of several different set-ups are presented. Silver nanocubes are first synthesized by the Polyol process, and deposited onto polystyrene thin films. By heating the substrate above polystyrene's $T_{g}$ the nanocubes can be embedded into the polymer. Copper Oxide is then grown on the cube's surface, with the surface coverage being controlled by the exposed surface area of the cube. Four set-ups were tried for the production of these types of particles, as described following.

\section{1 - Preparation of Substrates for Copper Oxide Growth}

Monolayers of silver nanocubes on polystyrene thin films were first prepared as templates for the growth of copper oxide partial shells. Silver nanocubes were 
synthesized and deposited onto polystyrene thin films, which were prepared by spincoating. Heating these substrates above the $\mathrm{T}_{\mathrm{g}}$ of polystyrene allowed the nanocubes to be partially embedded.

\subsection{1 - Synthesis of Silver Nanocubes}

Silver nanocubes with an edge length of $60 \mathrm{~nm}$ were synthesized for this work by the Polyol process. The extinction spectrum of these nanocubes in ethanol is displayed in Figure 3.1 A, with the dipolar mode at $469 \mathrm{~nm}$ and a multipolar mode at $346 \mathrm{~nm}$, indicative of a cubic structure. These nanocubes were large enough to also support a quadrupolar mode at $392 \mathrm{~nm}$. The size and morphology of these nanocubes were confirmed with the use of atomic force microscopy (Figure $3.1 \mathrm{~B}$ ).
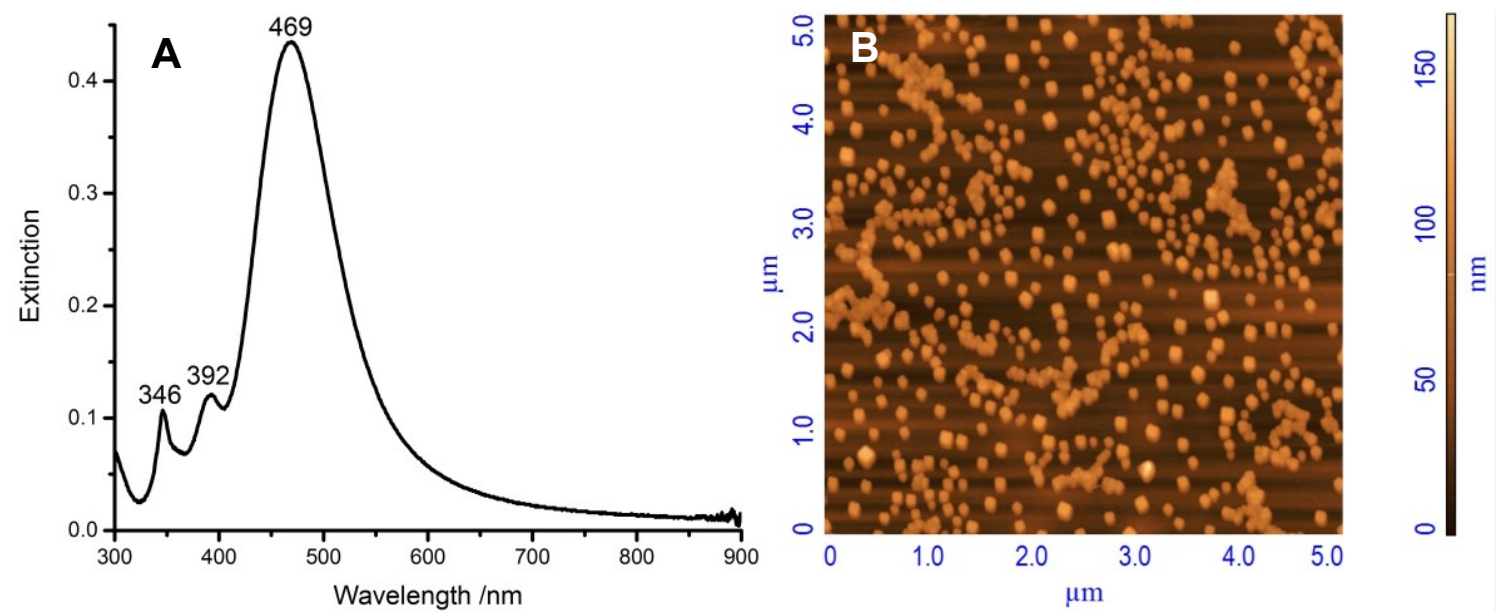

Figure 3.1: (A) UV-Vis spectra of silver nanocubes suspended in ethanol and (B) Atomic force micrograph of silver nanocubes.

\subsection{2 - Preparation of Polystyrene Thin Films}

Polystyrene thin films were prepared by spin-coating a $3 \mathrm{wt} \%$ solution in toluene onto glass slides. The substrates were characterized with atomic force microscopy to determine the roughness and thickness of the polystyrene films. In particular, the average root mean square roughness was determined to be $(1.08 \pm 0.13) \mathrm{nm}$. The thicknesses of the 
films were determined to be $\sim 130 \mathrm{~nm}$ thick, by cutting the film and measuring the topography by AFM. AFMs of a polystyrene thin-film and cut polystyrene thin-film can be seen in Figure 3.2 A and B, respectively.
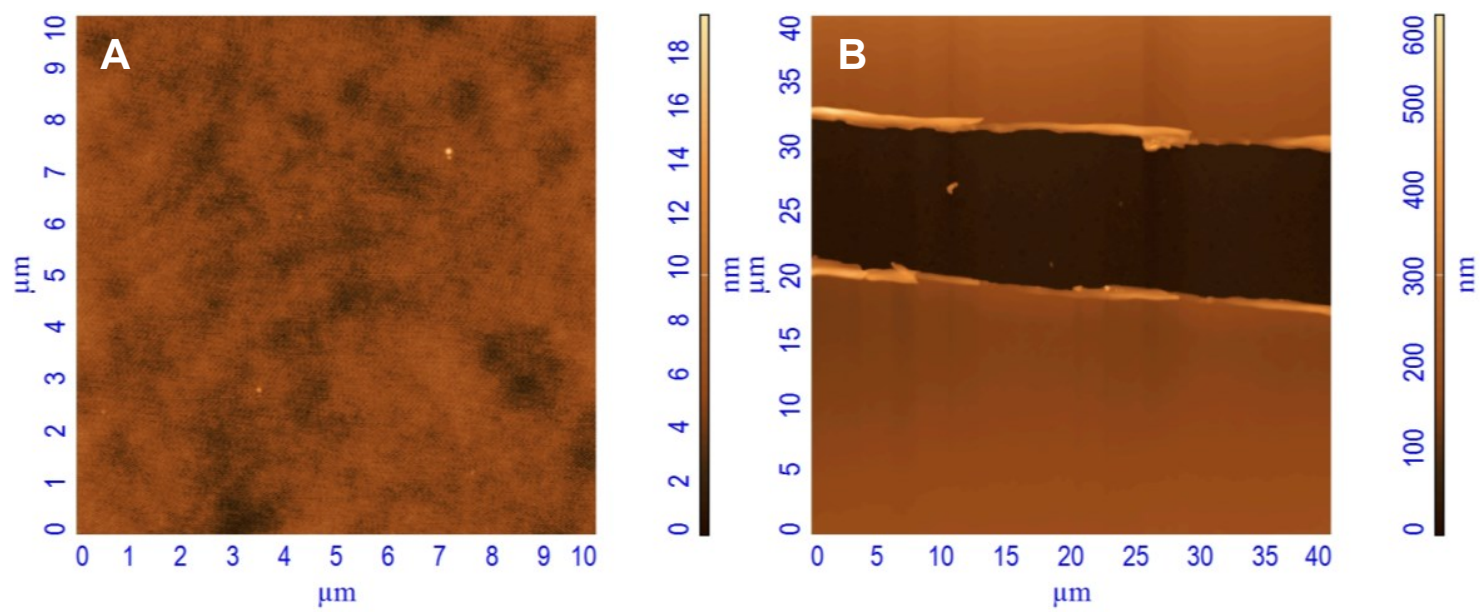

Figure 3.2: Atomic force micrographs of (A) polystyrene film prepared with $3 \mathrm{wt} \%$ polystyrene in toluene, (B) cut polystyrene film.

\subsection{3 - Langmuir-Blodgett Deposition of Silver Nanocubes}

The Langmuir-Blodgett method was used to deposit the silver nanocube sample onto the polystyrene substrates. Since copper oxide would be grown on these samples, it was necessary that the particles were adequately spaced and not aggregated on the substrate, so DOPC, a lipid spacer, was used, and the particles were transferred to the polystyrene at a low $(0.5 \mathrm{mN} / \mathrm{m})$ surface pressure.

These samples were characterized with UV-Vis (Figure $3.3 \mathrm{~A}$ ), and substrateinduced hybridization is observed in the spectra, with modes at $447 \mathrm{~nm}, 390 \mathrm{~nm}$ and 342 $\mathrm{nm}$, corresponding to the hybrid dipolar, hybrid quadrupolar and multipolar modes, respectively. In addition, coupling is not observed in the spectra, which indicates that the particles are spaced adequately, and not aggregated. In addition, AFMs were also obtained for these monolayers (Figure 3.3 B). From these images, it can be concluded 
that the nanocubes are mostly well separated; however, with the possibility of a few small aggregates.
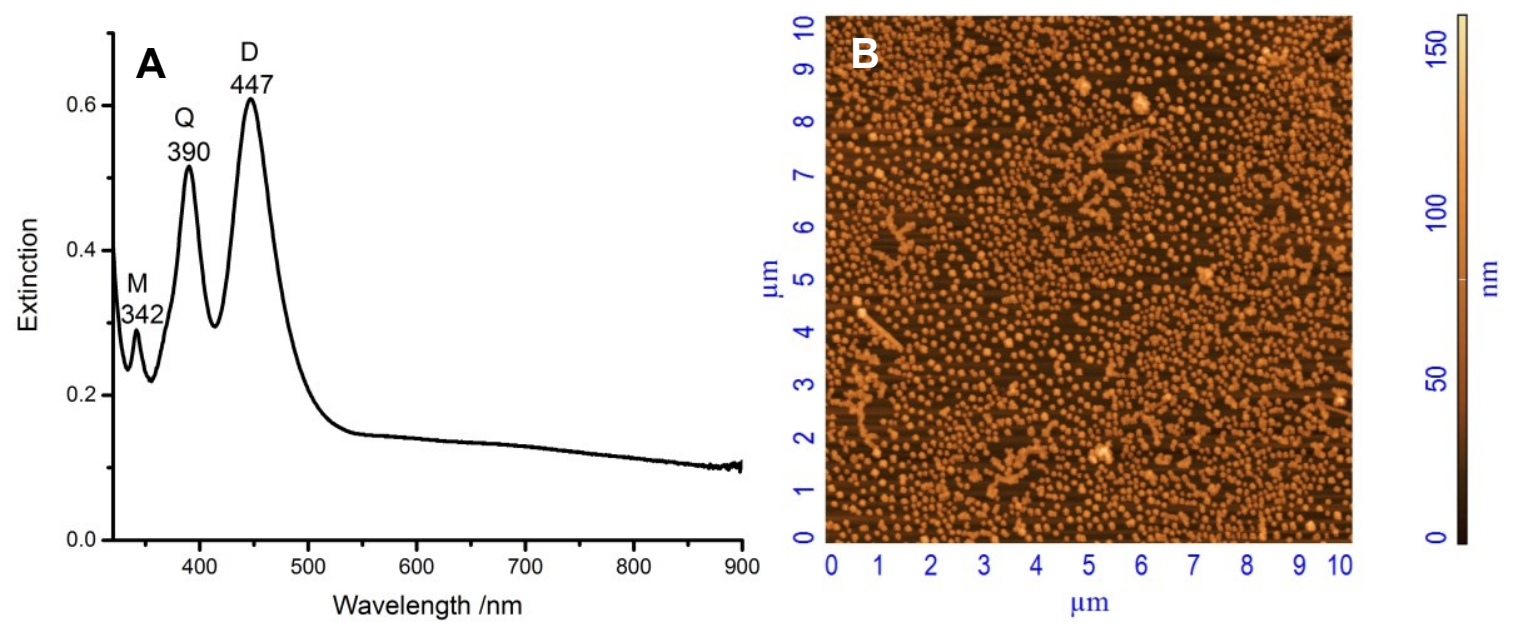

Figure 3.3: (A) UV-Vis of silver nanocubes supported on polystyrene thin-films and (B) Atomic force micrograph of silver nanocubes supported on polystyrene thin-films.

\subsection{4 - Embedding Silver Nanocubes into Polystyrene Thin Films}

The nanocubes were then partially embedded into the polystyrene thin films by heating the slides and monitoring the process by UV-Vis. Figure 3.4 displays the UV-Vis spectra of the samples over time, when heated at $115.5^{\circ} \mathrm{C}$. The substrate was removed from the hotplate every $10 \mathrm{~s}$, to acquire a spectrum, and then placed back on the hotplate to continue the embedding process. The hybrid D and hybrid Q modes were monitored throughout this process to determine the extent of embedded. Since it was desired that the nanocubes were embedded approximately half way, the process should be stopped once the hybrid dipolar mode has finished shifting and the hybrid quadrupolar mode has begun shifting in the UV-Vis spectra. In particular, it was observed that the hybrid dipolar mode, which is more sensitive to the substrate, begins to redshift slightly in the first time interval of $10 \mathrm{~s}$ and stops shifting by $60 \mathrm{~s}$, with the total shift being $\sim 25 \mathrm{~nm}$, from $446 \mathrm{~nm}$ initially to $471 \mathrm{~nm}$ (Figure 3.4). The hybrid quadrupolar mode, however, was observed to begin red shifting slightly by $30 \mathrm{~s}$ and continue to shift until the heating was stopped after 
$80 \mathrm{~s}$. The hybrid Q mode shifted from $394 \mathrm{~nm}$ to $408 \mathrm{~nm}$ during the embedding process (Figure 3.4). The embedded depth of the nanocubes was also confirmed to be $\sim 1 / 2$ way, with AFM measurements. In addition, during the embedding process, some of the nanocubes will tilt, ${ }^{117}$ which is observed by AFM, and will affect the subsequent shell growth. In particular, as the cubes can tilt such that they are corner-down, the shell can be grown over the exposed area, which would be the opposite corner.

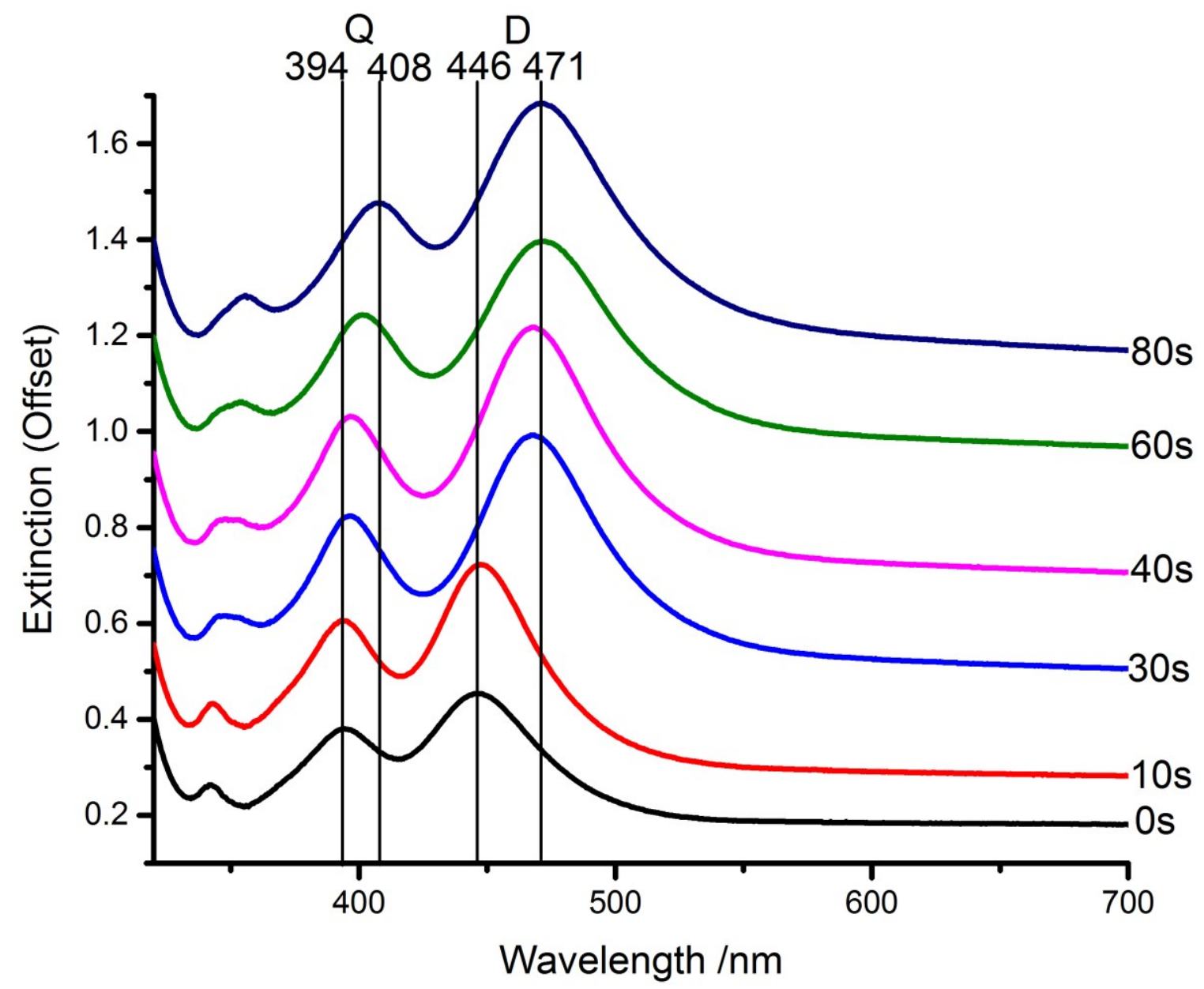

Figure 3.4: UV-Vis of silver nanocube monolayers on polystyrene at different time intervals during the embedding process at $115.5^{\circ} \mathrm{C}$.

\section{2 - Copper Oxide Growth}

Subsequently, these embedded samples were used for the growth of copper oxide shells on the exposed portion of the nanocubes. In particular, four set-ups were used to 
try to optimize the growth of the copper oxide shell. In addition, the concentrations and addition orders of the reagents were modified, and the resulting changes in produced morphologies were observed.

\subsection{1 - Theoretical Considerations}

FDTD calculations were performed by Dan Prezgot for a $70 \mathrm{~nm}$ silver cube which has a pyramidal partial shell. The pyramid has a $140 \mathrm{~nm}$ base and $70 \mathrm{~nm}$ height. Calculations were performed for various shell heights, specifically, $65 \mathrm{~nm}, 55 \mathrm{~nm}, 45 \mathrm{~nm}$ and $35 \mathrm{~nm}$, measured from the bottom of the cube (Figure 3.5). In the calculated spectra, hybridization is observed in all instances, with the best hybridization produced for the highest shell heights (lowest shell coverage). These results demonstrated the potential for hybridization in solution, and so it was desired to produce similar structures. As such, methods for the production of such type of particles were developed. 

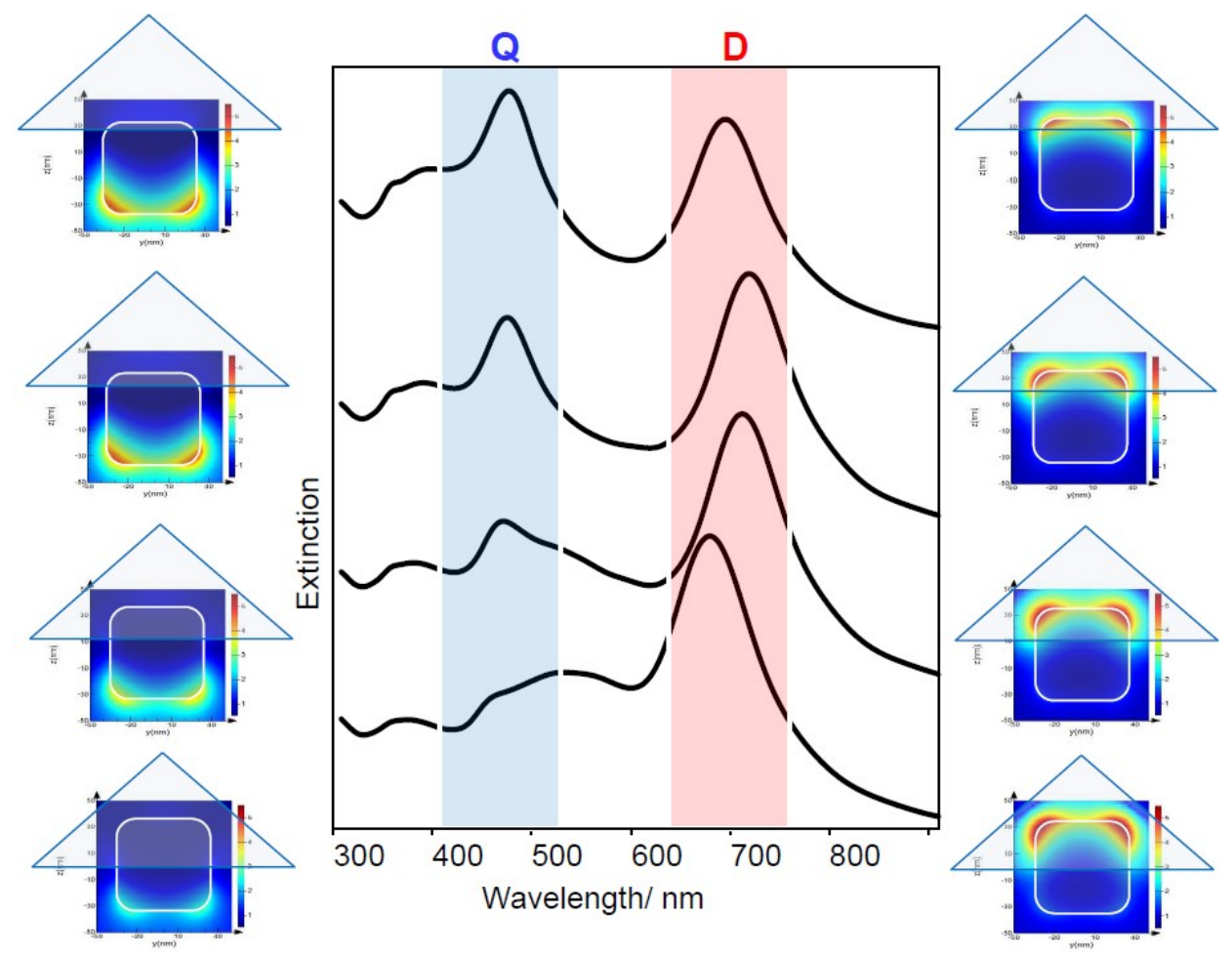

Figure 3.5: FDTD Calculated spectra of silver nanocubes with partial copper oxide shells with, 65 $\mathrm{nm}, 55 \mathrm{~nm}, 45 \mathrm{~nm}$ and $35 \mathrm{~nm}$ shell heights, measured from the bottom of the cube.

\subsubsection{Experimental Considerations}

In order to achieve the goal of controllably producing particles with a partial shell morphology, it was necessary to optimize the growth conditions. Particularly, several setups were used for the growth of copper oxide on the surface of silver nanocubes. Namely, four set-ups, the 'Teflon piece', 'Mechanical Stirrer', 'Puck', and the 'New Set-up' have been used for growth. Precisely, with the first three set-ups an investigation on the order of addition of reagents, namely the copper precursor (copper nitrate) and the reducing agent (hydrazine), was conducted. Specifically, either the copper nitrate or the hydrazine would be added to the reaction all at once, while the other would be added slowly, in intervals of 5 min throughout the reaction. Changing the order of addition of reagents has been reported to have an effect on the resulting morphologies of copper oxide produced, ${ }^{68}$ and it would be expected that a slow addition of the copper precursor or the reducing 
agent would alter the kinetics of the reaction; This could result in a great difference in produced structures, and was therefore of interest to investigate.

In addition, each of these sets (additions of copper nitrate or additions of hydrazine) were also tried in the presence and absence of lactic acid. Lactic acid has been used to direct the production of different facets,${ }^{74}$ and so it is expected that the produced structures will be different in its presence. Lastly, each of these were also tried for samples that were half-way embedded into polystyrene thin films and those that were supported on the polystyrene thin films, in order to produce core-shell morphologies that had a 'half-shell' or one bare face, respectively, and investigate their properties.

From the results produced with the use of the first three set ups (Mechanical Stirrer, Puck and Teflon Piece), the New Set-up was developed. With this set-up, attempts were made to optimize the growth of shells by changing the concentrations of $\mathrm{NaOH}$ and copper precursor, which have been shown to affect the sizes of the produced structures. ${ }^{48,76,77}$

\subsection{1 - Mechanical Stirrer}

The first set-up used to produce these core-partial shell nanoparticles was the Mechanical Stirrer (section 1.7.2). The result of adding copper nitrate or hydrazine throughout the reaction, with or without lactic acid, on samples which were embedded or not embedded, was investigated for this set-up. For samples where hydrazine was added over time, in the presence of lactic acid, it was observed that the solution would initially turn blue with addition of hydrazine, getting bluer over time, until 13-14 min where it turned green-yellow, and then orange-brown over the course of the reaction. In the absence of lactic acid, the progressions of colour changes were similar; however, the blue 
colour would result immediately after the copper precursor was added to the solution, and the colour changes would occur slightly more quickly, with the solution turning greenyellow by $8-9 \mathrm{~min}$. These results suggest that $\mathrm{Cu}(\mathrm{OH})_{2}$, or $\mathrm{Cu}(\mathrm{OH})_{4}{ }^{2-}$, which are blue in colour, ${ }^{35,68,73,87}$ are first forming, followed by the formation of copper oxide, $\mathrm{Cu}_{2} \mathrm{O}$, which can appear deep yellow or orange in solution. ${ }^{35,87}$ Brown could be the result of $\mathrm{CuO}$ formation. ${ }^{73}$

In contrast, in solutions where copper nitrate was added slowly, where sodium hydroxide was added first, then hydrazine, and then a solution of copper nitrate and lactic acid (if using) would be added, the colour was observed to first change to turn pale yellow, by $\sim 1 \mathrm{~min}$, and slowly become darker orange over time (for samples with and without lactic acid). In these cases, since there is more reducing agent in solution initially, the reduction to copper oxide (yellow/orange colour) ${ }^{35,87}$ seems to be able to occur more quickly.

\subsection{2 - Slow Addition of Hydrazine, with Lactic Acid}

Using the 'Mechanical Stirrer', shells were grown, where aliquots of hydrazine were added every 5 min throughout the reaction (in the presence of lactic acid). From the UV-Vis spectra of the samples after shell growth, modes appear red-shifted of the initial dipole modes at $652 \mathrm{~nm}$ and $654 \mathrm{~nm}$, for embedded (Figure 3.6 A) and supported samples (Figure 3.6 B), respectively. This emergence of a mode is consistent with shell growth. 

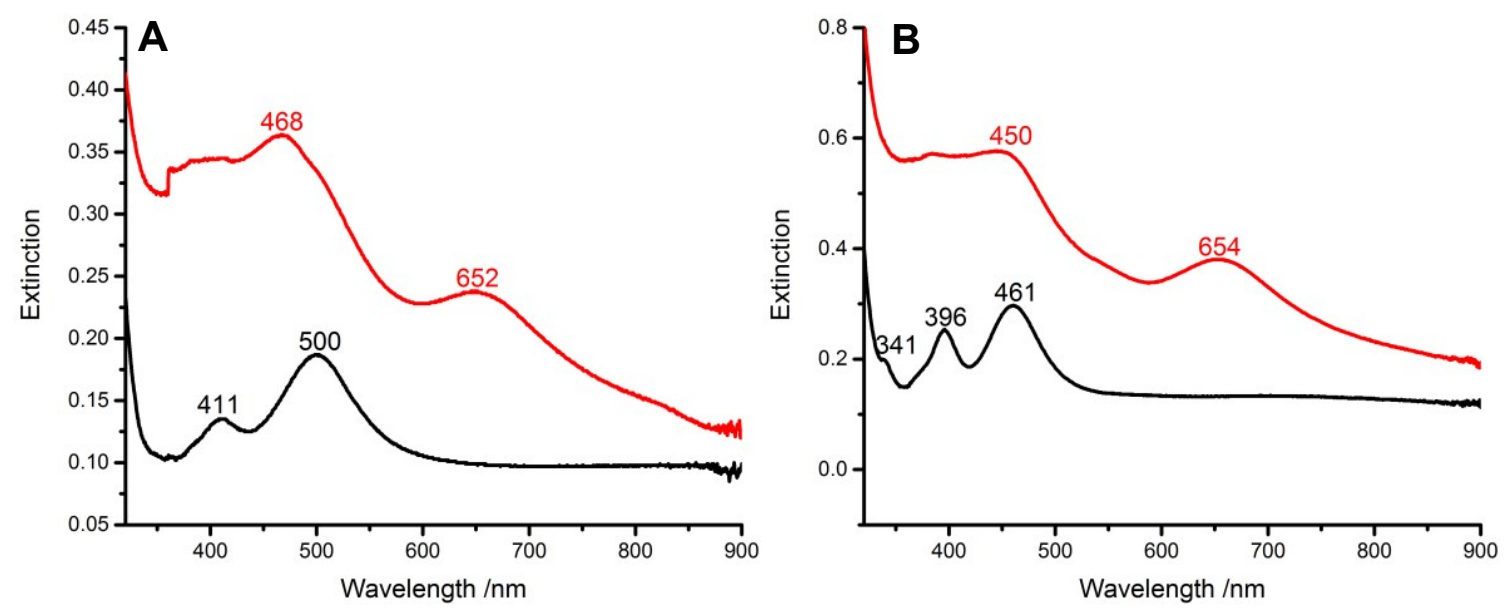

Figure 3.6: Silver nanocube monolayers before (Black) and after copper oxide growth (Red) for (A) embedded (slide 14_4) and (B) supported (slide 1_3) samples.

Subsequent removal of these samples and characterization with TEM revealed shells which had grown significantly, and were porous and disorganized in structure, for both the initially embedded (Figure 3.7 A1-A3) and initially supported (Figure 3.7 B1B3) samples. With the use of Image ${ }^{120}$ data processing software, the shell thicknesses were estimated to be $67 \mathrm{~nm} \pm 20 \mathrm{~nm}$ and $64 \mathrm{~nm} \pm 20 \mathrm{~nm}$ for those produced with embedded and supported particles. This indicates that the same thickness of copper oxide is obtained for both samples prepared from embedded and supported nanocubes. The UV-Vis spectra of these samples had hybridization of the modes for both samples, with D and Q modes at $662 \mathrm{~nm}$ and $480 \mathrm{~nm}$ for the initially embedded sample (Figure 3.7, right, red) and at $666 \mathrm{~nm}$ and $470 \mathrm{~nm}$ for the initially supported sample (Figure 3.7, right, black), respectively. In this sample, hybridization is better for the sample prepared from supported cubes, which could be due to the embedded nanoparticles tilting during the embedding, which could cause less conformal shell growth. 

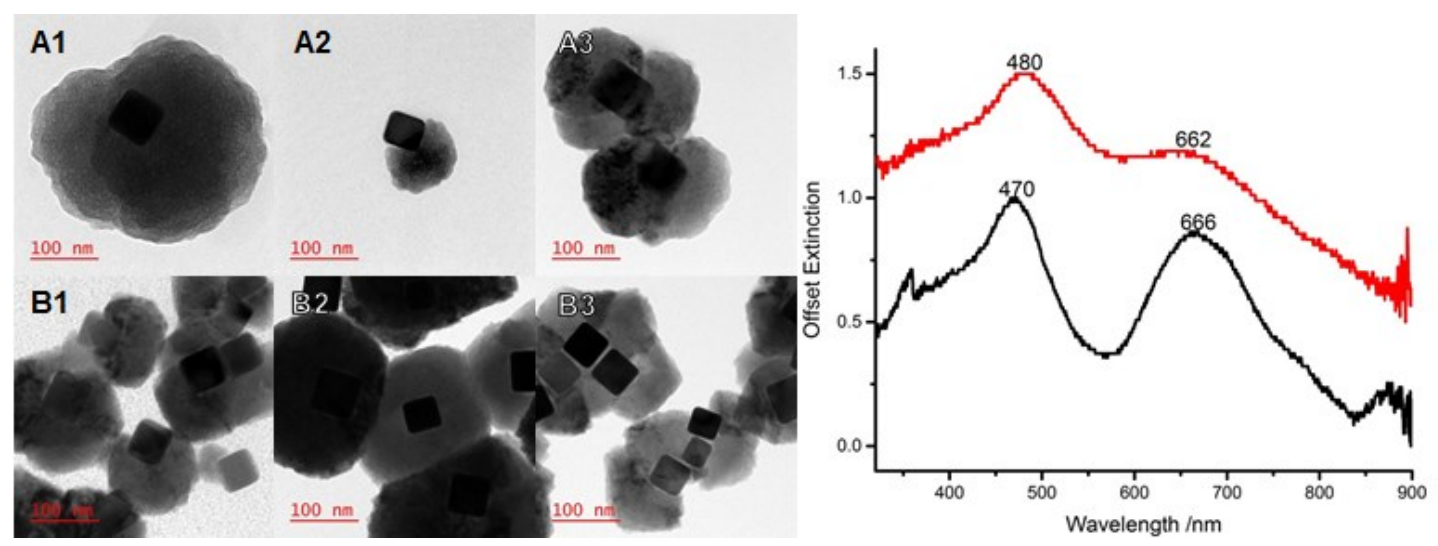

Figure 3.7: (Left) Transmission electron micrographs of shells grown with 10 additions of hydrazine, with lactic acid from (A1-A3) embedded (slide 14_4) and (B1-B3) supported (slide 1_3) samples (Right) In-solution spectra of samples prepared from (Red) embedded (slide 14_4), (Black) supported (slide 1_3) samples

\subsection{3 - Slow Addition of Copper, with Lactic Acid}

Samples were also prepared with the additions of aliquots of a solution with copper nitrate and lactic acid throughout the shell synthesis, in intervals of $5 \mathrm{~min}, 10$ times. After shell growth, new modes have emerged in the spectra at $647 \mathrm{~nm}$ for the embedded sample (Figure $3.8 \mathrm{~A}$ ), and $624 \mathrm{~nm}$ for the supported sample (Figure $3.8 \mathrm{~B}$ ), indicating shell growth.
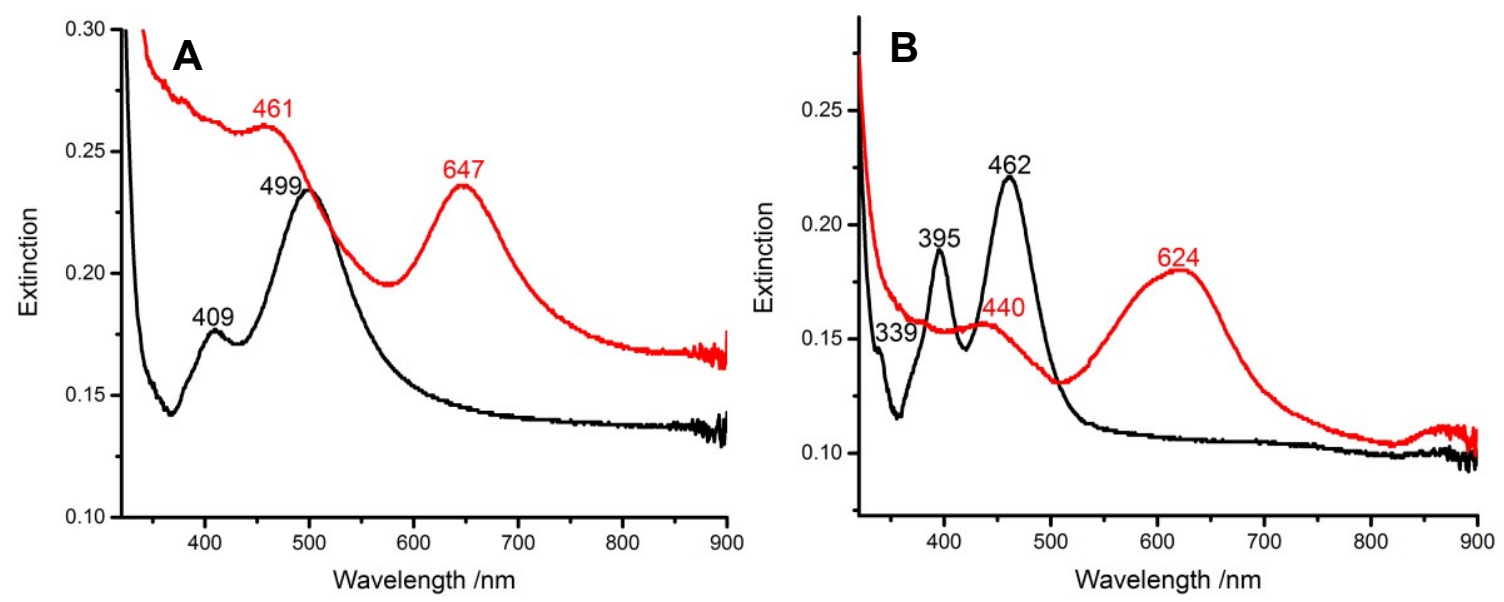

Figure 3.8: Silver nanocube monolayers before (Black) and after copper oxide growth (Red) for (A) embedded (slide 14_2) and (B) supported (slide 1_5) samples. 
Removal of these samples and subsequent TEM images indicated that the produced shells were smoother overall and smaller (Figure 3.9 A1-A3, B1-B3) than the ones produced by adding hydrazine throughout (Figure 3.9 A1-A3, B1-B3). The partial shell morphology is observable for the samples that were embedded in the polystyrene during the shell growth (Figure 3.9 A1, A2). As well, in some of the images it is observed that the shell appears to have nucleated at many points, which have grown into each other (Figure 3.9 A3, B1-B3). The shell thicknesses were estimated to be $47 \mathrm{~nm} \pm 20 \mathrm{~nm}$ and $32 \mathrm{~nm} \pm 8 \mathrm{~nm}$ for the samples prepared from embedded and supported silver nanocubes, respectively, with the use of ImageJ data processing software. These thickness results suggest that the thicknesses of copper oxide obtained for initially embedded and supported nanocubes are comparable; however, measurement of the shell thickness provided results with large uncertainty, due to the inconsistent shell morphologies. In addition, the UV-Vis spectra show hybridization for both samples, with improved hybridization for the initially embedded sample.
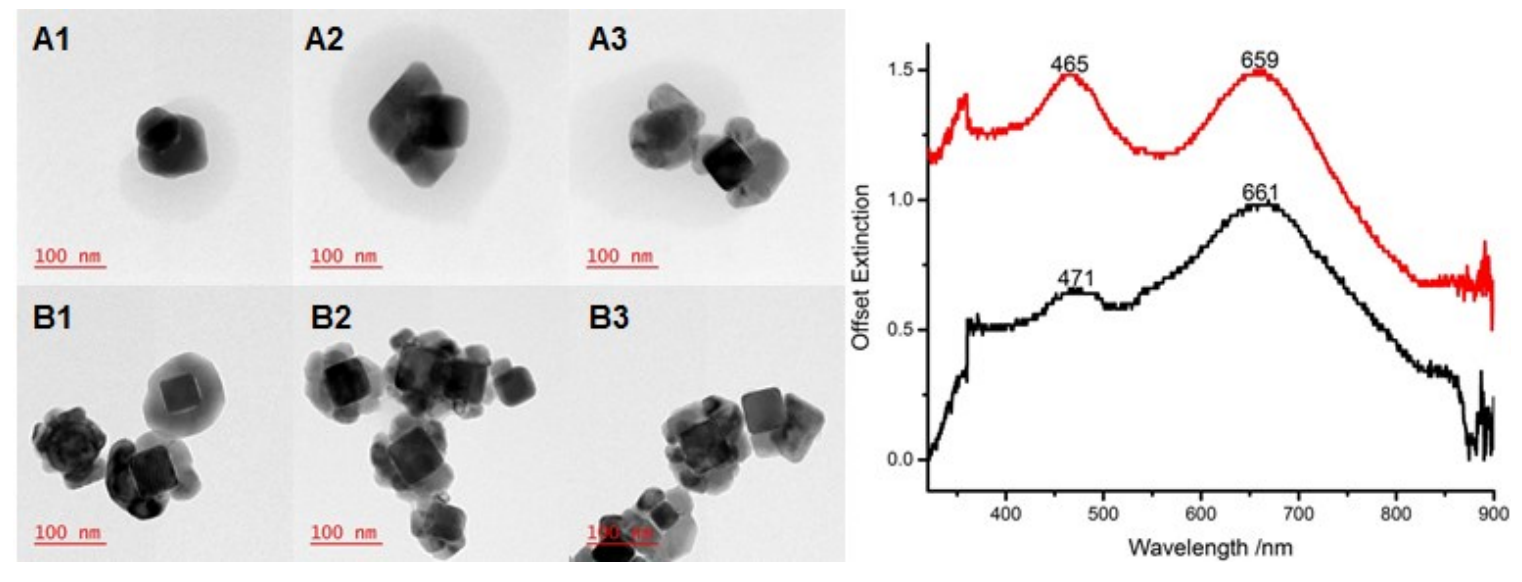

Figure 3.9: Transmission electron micrographs of shells grown with 10 additions of copper, with lactic acid from (A1-A3) embedded (slide 14_2) and (B1-B3) supported (slide 1_5) samples. (Right) Insolution spectra of samples prepared from (Red) embedded (slide 14_2), (Black) supported (slide 1_5) samples. 


\subsection{4 - Slow Addition of Hydrazine, without Lactic Acid}

Samples were prepared with additions of hydrazine throughout the reaction, with $1 \mathrm{~mL}$ of the solution added every $5 \mathrm{~min}$ (10 times), without lactic acid. UV-Vis spectra of the samples after synthesis, have new modes red-shifted of the initial dipole peaks, indicating growth of copper oxide shells. Notably, these modes are observed at $681 \mathrm{~nm}$ for the embedded samples (Figure $3.10 \mathrm{~A}$ ), and $684 \mathrm{~nm}$ for the supported samples (Figure $3.10 \mathrm{~B})$.
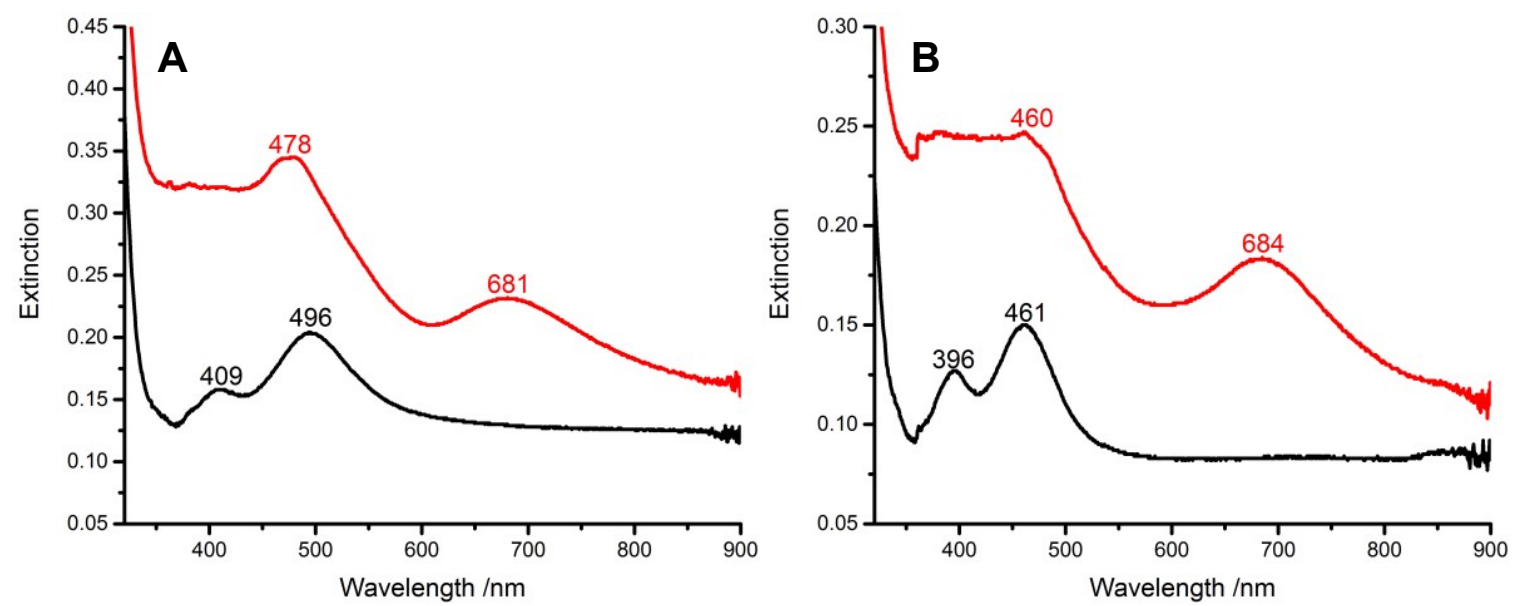

Figure 3.10: Silver nanocube monolayers before (Black) and after copper oxide growth (Red) for (A) embedded (slide 14_3) and (B) supported (slide 11_4) samples.

Removal of these samples from the substrates and acquisition of TEMs revealed mostly cubic-shaped shell growth, for both the samples which were embedded (Figure 3.11 A1-A3) and those supported (Figure 3.11 B1-B3). The $\{100\}$ facet is the lowest energy facet for $\mathrm{Cu}_{2} \mathrm{O},{ }^{70}$ and so in the absence of capping/structural guiding agents, cube shapes are obtained. The thicknesses were measured with ImageJ data processing software and determined to be $48 \mathrm{~nm} \pm 16 \mathrm{~nm}$ and $48 \mathrm{~nm} \pm 18 \mathrm{~nm}$, for the shells grown on embedded and supported silver nanocube samples, respectively, indicating consistent thicknesses between samples. 
For both the core-shell structures prepared from embedded and supported nanocubes, hybridization is observed in the UV-Vis spectra (Figure 3.11 right). For the samples initially embedded in the polystyrene, the D and Q modes are located at $673 \mathrm{~nm}$ and $486 \mathrm{~nm}$, respectively; however, for the samples prepared from the supported silver nanocubes, the D and Q modes are located at $697 \mathrm{~nm}$ and $486 \mathrm{~nm}$, respectively. Since $\mathrm{Cu}_{2} \mathrm{O}$ is a higher refractive index material, additional growth of these shells, which occurs on the supported samples relative to the embedded samples, could result in further red shift of the modes, as observed.
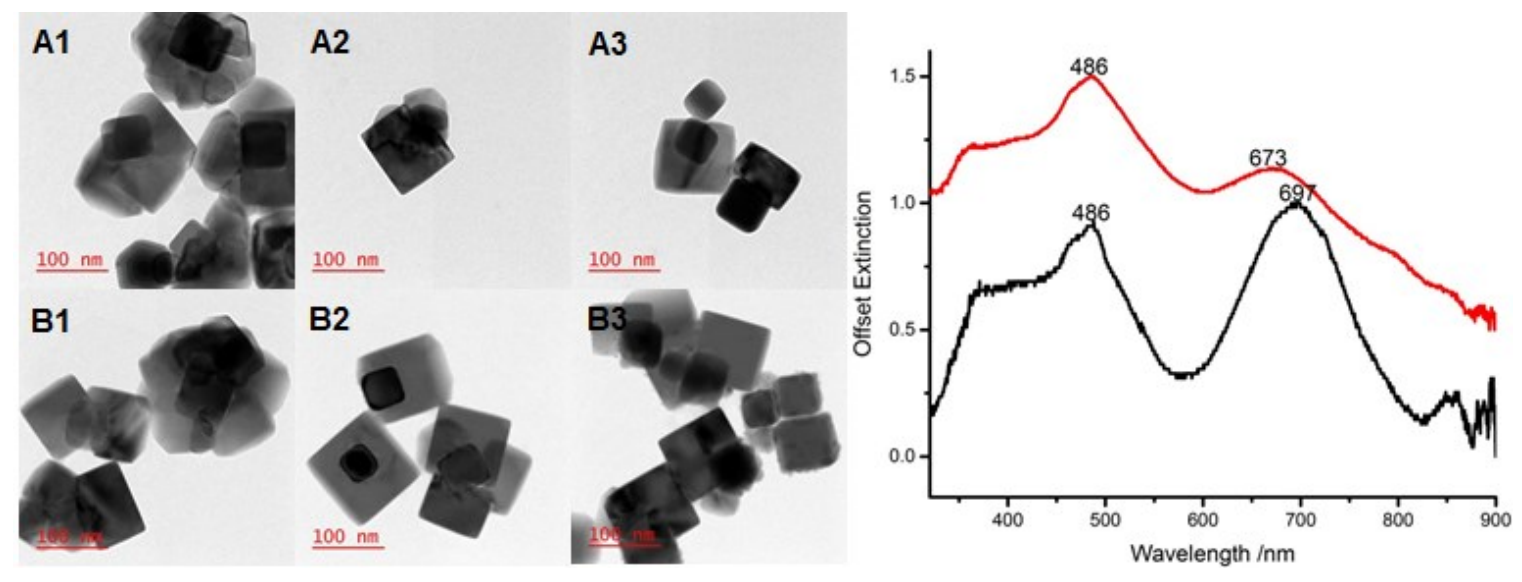

Figure 3.11: (Left) Transmission electron micrographs of shells grown with 10 additions of hydrazine, without lactic acid from (A1-A3) embedded (slide 14_3) and (B1-B3) supported (slide 11_4). (Right) In-solution spectra of samples prepared from (Red) embedded (slide 14_3), (Black) supported (slide 11_4) samples.

\subsection{5 - Slow Addition of Copper, without Lactic Acid}

Samples to which only a solution of copper nitrate had been added throughout the reaction, with additions every 5 min (10 times), were observed to have the development of modes red-shifted of the initial dipole peaks. In particular, for the embedded samples of silver nanocubes, this mode is located at $692 \mathrm{~nm}$ (Figure $3.12 \mathrm{~A}$ ); for supported samples, this mode is located at $657 \mathrm{~nm}$ (Figure $3.12 \mathrm{~B}$ ). 

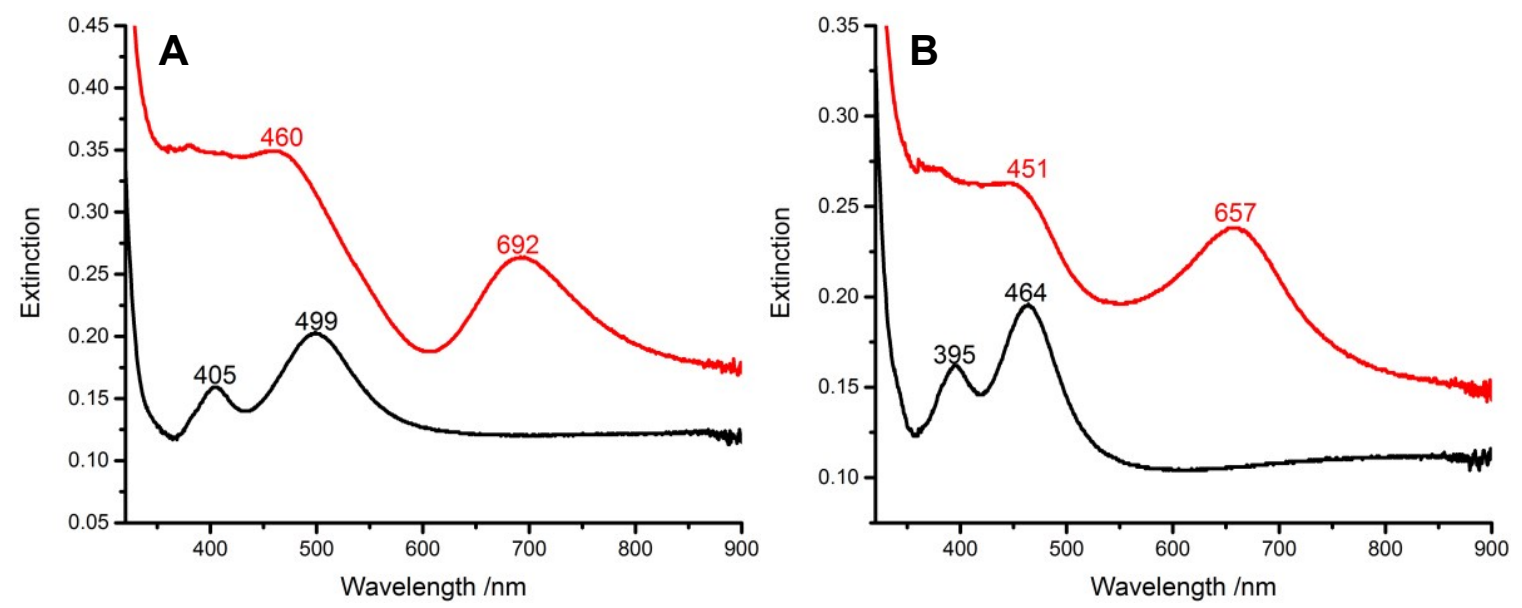

Figure 3.12: Silver nanocube monolayers before (Black) and after copper oxide growth (Red) for (A) embedded (slide 14_1) and (B) supported (slide 11_3) samples.

These samples were subsequently removed and re-suspended in toluene. TEMs show that on many of the nanocubes, the copper oxide shells have nucleated at several points, growing as semi-cubic and pyramidal structures, and have further grown into each other forming the shells (Figure 3.13 A1-A3, B1-B3). Partial shells can be observed in the samples which were embedded in the polystyrene during the copper oxide growth (Figure 3.13 A2, A3). The thicknesses of the produced copper oxide shells were determined to be $48 \mathrm{~nm} \pm 15 \mathrm{~nm}$ and $44 \mathrm{~nm} \pm 11 \mathrm{~nm}$ for those prepared from embedded and supported samples, respectively, indicating comparable thicknesses between samples.

In addition, hybridization is observed in the UV-Vis spectra for both the samples which were embedded (Figure 3.13, right, red) and for those that were supported (Figure 3.13, right, black) during the shell growth. The D and Q modes are located at $701 \mathrm{~nm}$ and $479 \mathrm{~nm}$ for the particles embedded during synthesis, and $684 \mathrm{~nm}$ and $466 \mathrm{~nm}$ for the particles supported during shell growth, respectively. 

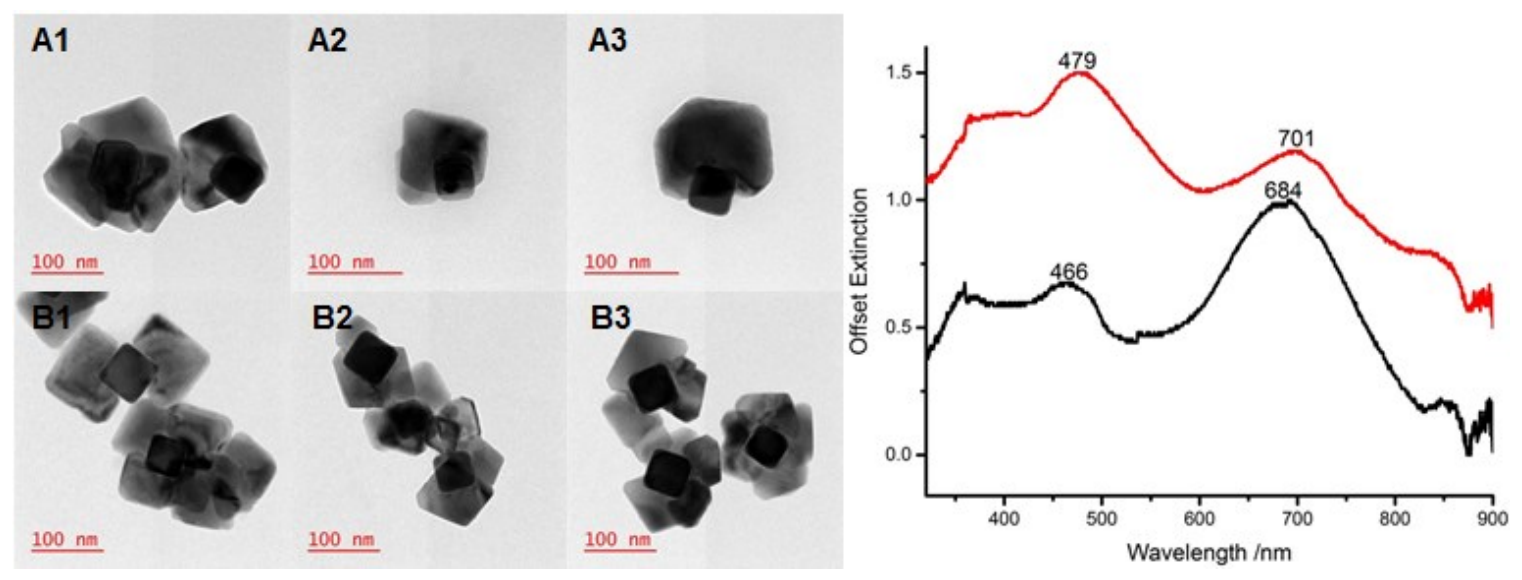

Figure 3.13: (Left) Transmission electron micrographs of shells grown with 10 additions of copper, without lactic acid from (A1-A3) embedded (slide 14_1) and (B1-B3) supported (slide 11_3). (Right) In-solution spectra of samples prepared from (Red) embedded (slide 14_1) and (Black) supported (slide 11_3) samples.

\subsection{6 - Concluding Statements on the Mechanical Stirrer}

In conclusion, the use of the Mechanical Stirrer for the production of core-partial shell silver-copper oxide nanoparticles showed promising results for the use of such types of set-ups to produce these types of anisotropic structures. The order and rates of addition of the reagents was modified, during the growth of copper oxide shells for embedded and supported silver nanocube monolayers. In addition, these syntheses were also tried with and without the addition of lactic acid.

To summarize, in the presence of lactic acid, the slow addition of hydrazine produced larger and more porous shells than those produced with the slow addition of copper precursor. In the absence of lactic acid, the slow addition of hydrazine produced mostly cubic shaped shells, while the slow addition of copper precursor produced semicubic and pyramidal shaped shell pieces. For both, nucleation often appeared to have happened on multiple points of the particle, and grown into one another. In addition, the produced core-partial shell nanoparticles display hybridization in the in-solution spectra, which is consistent with the theoretical expectation for these types of particles. 
Although this set-up showed promising initial results, it can be improved by using a set-up which allows the spin speed to be changed, which would allow the effect of the stirring rate to also be investigated. In addition, a steel rod with a slide epoxied to it was used as the sample support, and was difficult to thoroughly clean, which is an issue that must be addressed. As such, to overcome these issues, another set-up, the 'Puck', was attempted.

\subsection{1 - Puck}

With the 'Puck' set-up, samples were again produced, with slow additions of copper nitrate or hydrazine, in the presence and absence of lactic acid, and on samples which were embedded in or supported on polystyrene thin films. The solution for samples to which hydrazine was added throughout the reaction (with lactic acid) initially turned blue after the addition of hydrazine, and subsequently yellow at $\sim 11 \mathrm{~min}$ and orange at $\sim 15$ min. For the samples with hydrazine added without lactic acid, the solution turned blue directly after the addition of copper nitrate, then turned green by $\sim 8 \mathrm{~min}$, yellowbrown by $\sim 14 \mathrm{~min}$, and orange by $\sim 27 \mathrm{~min}$. The blue colour ${ }^{35,68,73,87}$ could be the production of $\mathrm{Cu}(\mathrm{OH})_{2}$ or $\mathrm{Cu}(\mathrm{OH})_{4}{ }^{2-}$. The subsequent colour change to orange is attributed to $\mathrm{Cu}_{2} \mathrm{O} .^{35,87}$

For samples to which copper was added throughout the reaction (with and without lactic acid), the solutions turned yellow by $\sim 1 \mathrm{~min}$, and subsequently turned orange over the course of the reaction. This result again suggests that with the samples to which all the reducing agent was added initially, the formation of $\mathrm{Cu}_{2} \mathrm{O}$ occurs more quickly, as indicated by the yellow and orange colours. ${ }^{35,87}$ 


\subsection{2 - Slow Addition of Hydrazine, with Lactic Acid}

With this set-up, samples were prepared with the addition of hydrazine every 5 min (10 times), over the course of the reaction, in the presence of lactic acid. From the $\mathrm{UV}-\mathrm{V}$ is spectra of the substrates after the reaction, new peaks are observed to have developed, red-shifted of the initial dipole mode, at $649 \mathrm{~nm}$ for embedded samples (Figure $3.14 \mathrm{~A}$ ) and at $572 \mathrm{~nm}$ for supported samples (Figure $3.14 \mathrm{~B}$ ), indicating the growth of copper oxide shells on the surface.
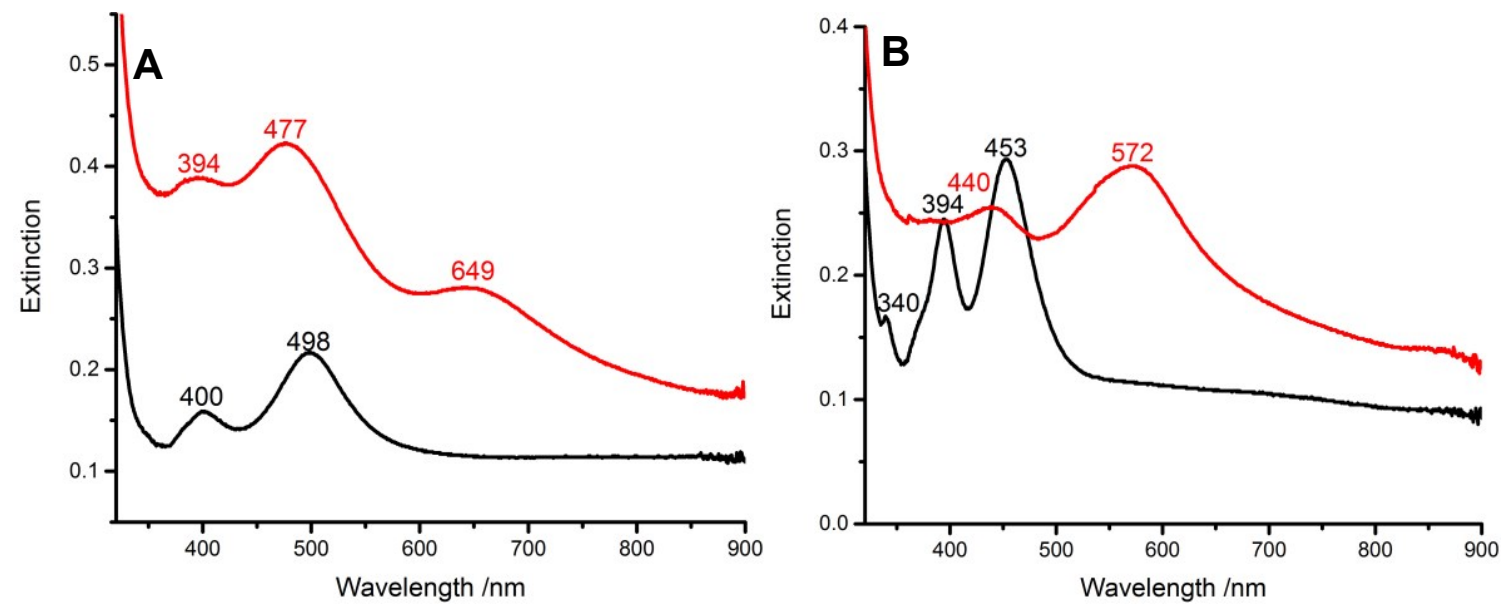

Figure 3.14: Silver nanocube monolayers before (Black) and after copper oxide growth (Red) for (A) embedded (slide 13_4) and (B) supported (slide 1_2) samples.

Removal of these samples revealed shells which appeared to be porous in the TEMs (Figure 3.15 A1-A3, B1-B3). The samples that were embedded during the growth process are observed have produced structures which have a partial shell, with the exposed portion of the nanocube visible (Figure 3.15 A1-A3). Nucleation appears to have occurred at many areas on the surface of the cubes, especially in the sample in which the silver nanocubes were supported on the polystyrene during growth (Figure 3.15 B1-B3). The thickness of the copper oxide layer was measured with ImageJ data processing software and found to be $80 \mathrm{~nm} \pm 28 \mathrm{~nm}$ and $35 \mathrm{~nm} \pm 6 \mathrm{~nm}$ for the structures prepared from embedded and supported nanocubes, respectively. This result indicates that thicker 
shells are produced for the embedded samples, which could be due to the nucleation at many points on the surface of the supported nanocubes, leading to thinner shells relative to the embedded nanocubes, where the shell only grow off of one area (Figure $3.15 \mathrm{~A}, \mathrm{~B}$ ). UV-Vis spectra for these samples were also obtained and the development of some hybridization for the samples which were prepared from embedded silver nanocubes was observed, with a peak at $487 \mathrm{~nm}$ and a shoulder at $654 \mathrm{~nm}$ (Figure 3.15, right, red). The UV-Vis spectra obtained in-solution from the samples prepared from supported silver nanocubes resembles a red shifted cube spectra, which could be explained by having one bare face, with all other faces coated in copper oxide, which redshifts the spectra, with a main peak at $618 \mathrm{~nm}$ (Figure 3.15, right, black).
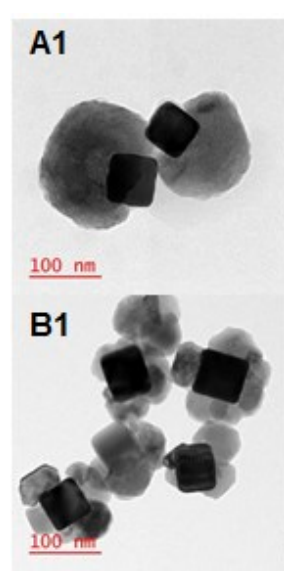
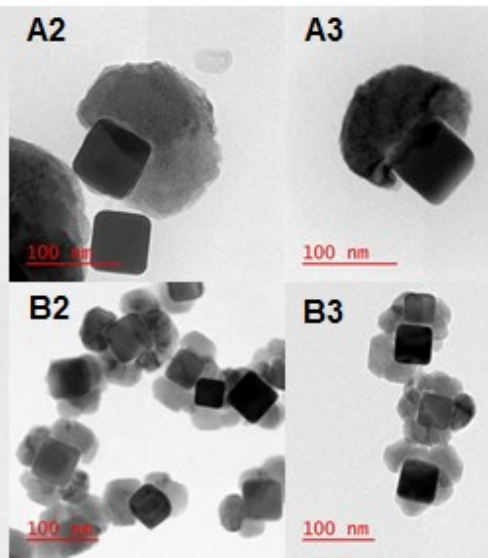

A3

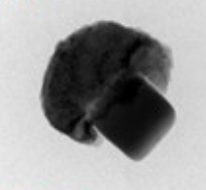

$100 \mathrm{~nm}$

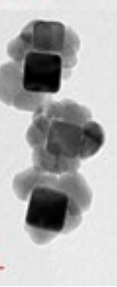

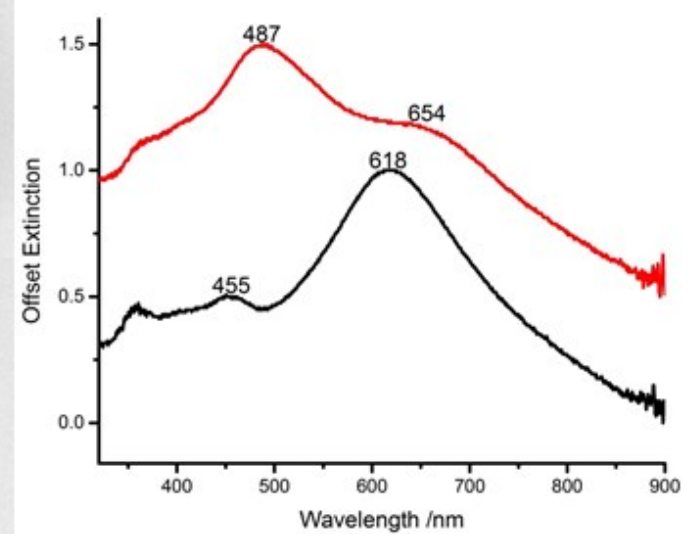

Figure 3.15: (Left) Transmission electron micrographs of shells grown with 10 additions of hydrazine, with lactic acid from (A1-A3) embedded (slide 13_4) and (B1-B3) supported (slide 1_2) samples, (Right) In-solution spectra of samples prepared from (Red) embedded (slide 13_4), (Black) supported (slide 1_2) samples.

\subsection{3 - Slow Addition of Copper, with Lactic Acid}

Samples were prepared with the addition of aliquots of a solution of copper nitrate and lactic acid, every $5 \mathrm{~min}$, throughout the reaction. After the reaction had been carried out, the UV-Vis spectra indicated that growth had occurred, with new modes emerging at 
$640 \mathrm{~nm}$ for the embedded sample (Figure $3.16 \mathrm{~A}$ ) and $593 \mathrm{~nm}$ for the supported sample (Figure $3.16 \mathrm{~B})$.
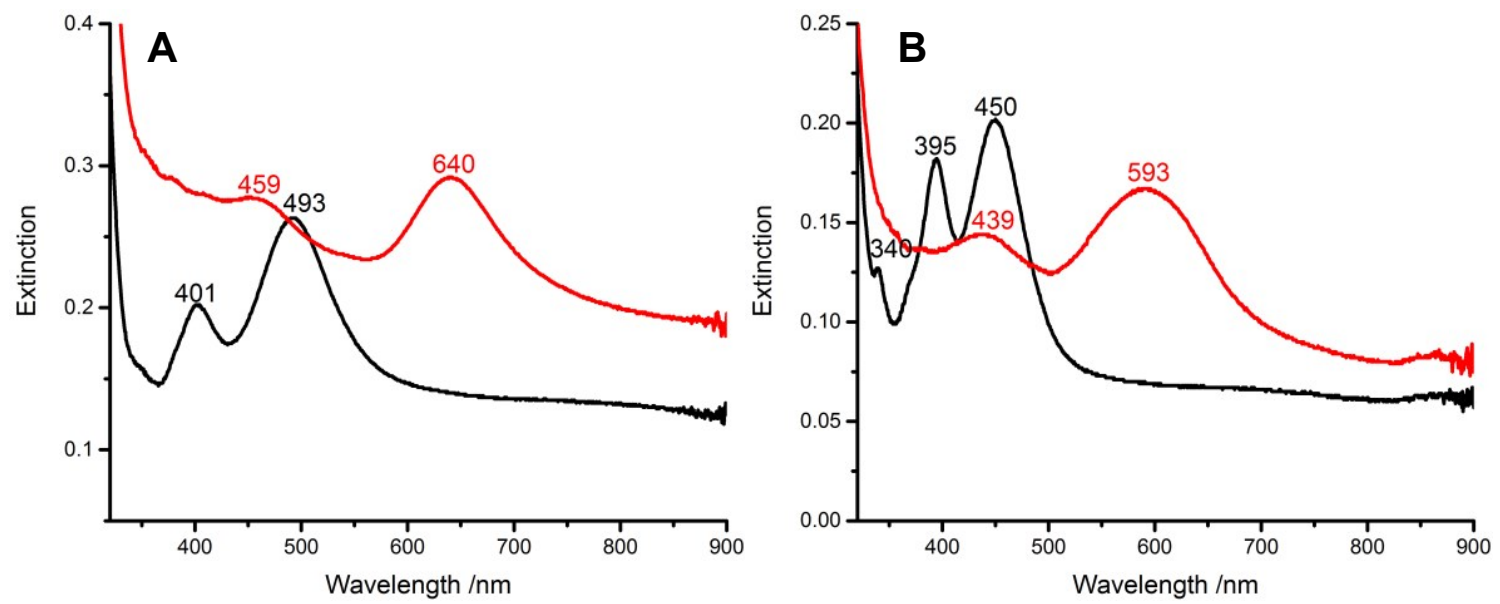

Figure 3.16: Silver nanocube monolayers before (Black) and after copper oxide growth (Red) for (A) embedded (slide 13_2) and (B) supported (slide 1_4).

The morphologies of the shells are observed with TEM, and these samples are observed to have many nucleation points on the cube's surface for both samples which were embedded (Figure 3.17 A1-A3) and supported (Figure 3.17 B1-B3) during growth. The observed shell structure is composed of many rounded units, which appear to be mostly attached to the faces, arranged like 'petals'. The thicknesses of these shell structures were determined with ImageJ data processing software. The thicknesses were $37 \mathrm{~nm} \pm 9 \mathrm{~nm}$ for shells grown on embedded nanocubes and $28 \mathrm{~nm} \pm 6 \mathrm{~nm}$ for shells grown on supported nanocubes.

The shapes of the resulting UV-Vis spectra are similar to those obtained for the corresponding samples with additions of hydrazine (with lactic acid) (Figure 3.17 right). Specifically, the sample that was prepared from embedded silver nanocubes shows some hybridization, with D and Q modes at $660 \mathrm{~nm}$ and $486 \mathrm{~nm}$, respectively; in contrast, the sample prepared from supported silver nanocubes's spectra in-solution resembles a red- 
shifted silver nanocube spectra, which could be due to copper oxide covering most of the nanocubes' surface.

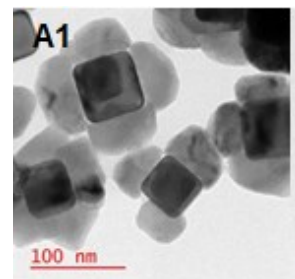

B1

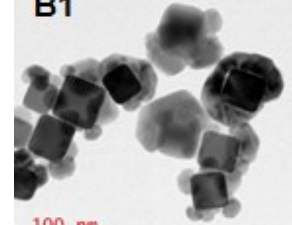

$\underline{100 \mathrm{~nm}}$
A2

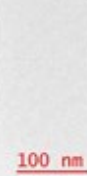

B2
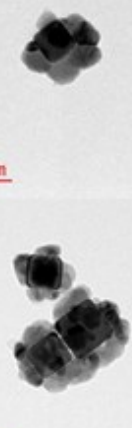

$\underline{100 \mathrm{~nm}}$

Figure 3.17: Transmission electron micrographs of shells grown with 10 additions of copper, with lactic acid from (A1-A3) embedded (slide 13_2) and (B1-B3) supported (slide 1_4) samples, (Right) Insolution spectra of samples prepared from (Red) embedded (slide 13_2), (Black) supported (slide 1_4) samples.

\subsection{4 - Slow Addition of Hydrazine, without Lactic Acid}

Samples were made whereby hydrazine was added to the solutions, every $5 \mathrm{~min}$ (10 times), throughout the reaction, in the absence of lactic acid. For these samples, it is observed that modes develop at $653 \mathrm{~nm}$ for the samples embedded (Figure $3.18 \mathrm{~A}$ ) and $687 \mathrm{~nm}$ for the supported samples (Figure $3.18 \mathrm{~B}$ ), after growth of copper oxide shells.
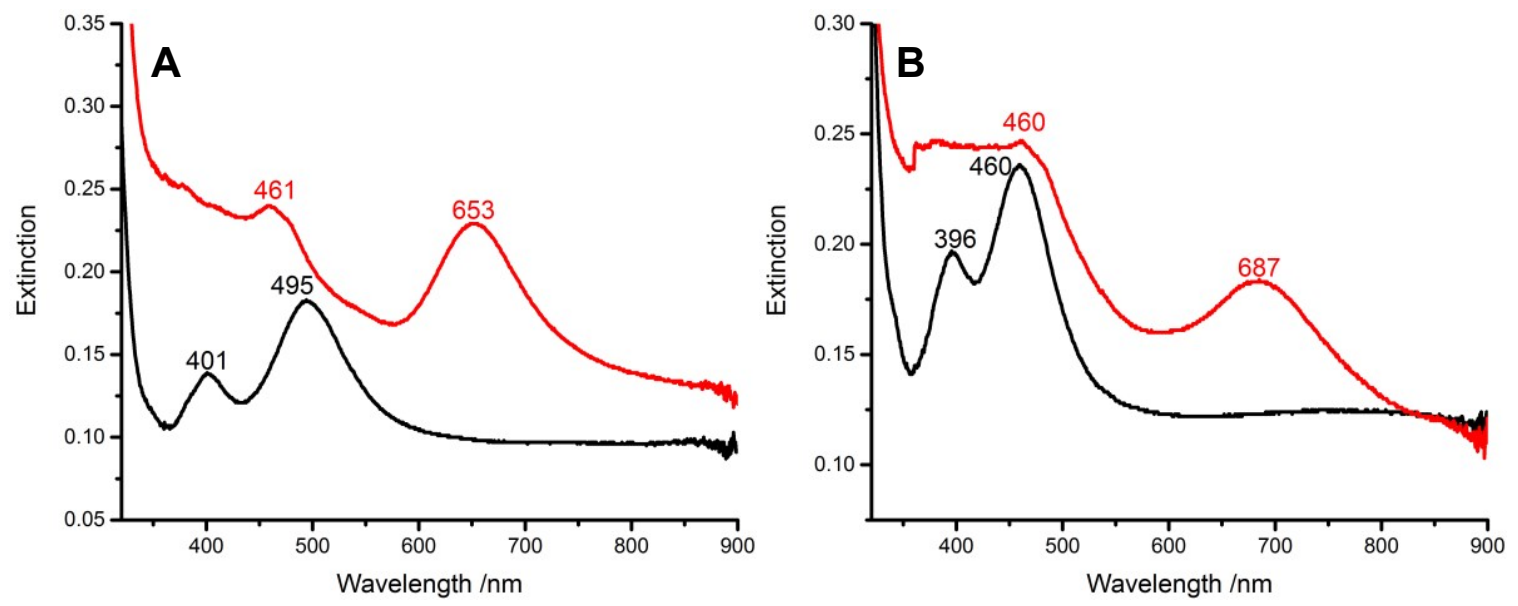

Figure 3.18: Silver nanocube monolayers before (Black) and after copper oxide growth (Red) for (A) embedded (slide 13_3) and (B) supported (slide 11_2). 
TEM images revealed shell structures which had been grown from many nucleation points (Figure 3.19 A1-A3, B1-B3). The partial-shell morphology is observable in the sample which was partially embedded during growth (Figure 3.19 A2). The shells are composed of many portions which have grown and coalesced into one another, some of which are cubic in shape (Figure 3.19 A2, A3). This shell composed of many pieces is also observed in the sample which was supported during synthesis (Figure 3.19 B1-B3). The thicknesses of the shells grown were found to be $43 \mathrm{~nm} \pm 19 \mathrm{~nm}$ and $35 \mathrm{~nm} \pm 13 \mathrm{~nm}$ for those prepared from embedded and supported samples, respectively, with the use of ImageJ data processing software. These thickness values indicate comparable thicknesses of copper oxide were grown between samples.

UV-Vis spectra were also obtained for these samples, with the sample that was embedded during shell growth showing hybridization, with peaks at $673 \mathrm{~nm}$ and $471 \mathrm{~nm}$ (Figure 3.19, right, red). In contrast, the core-shell particles which were prepared with supported silver nanocubes show a peak at $684 \mathrm{~nm}$ (Figure 3.19, right, black), which could be due to the copper oxide coating covering all but one face, instead of only half the particle, as is the case for those prepared from embedded nanocubes.
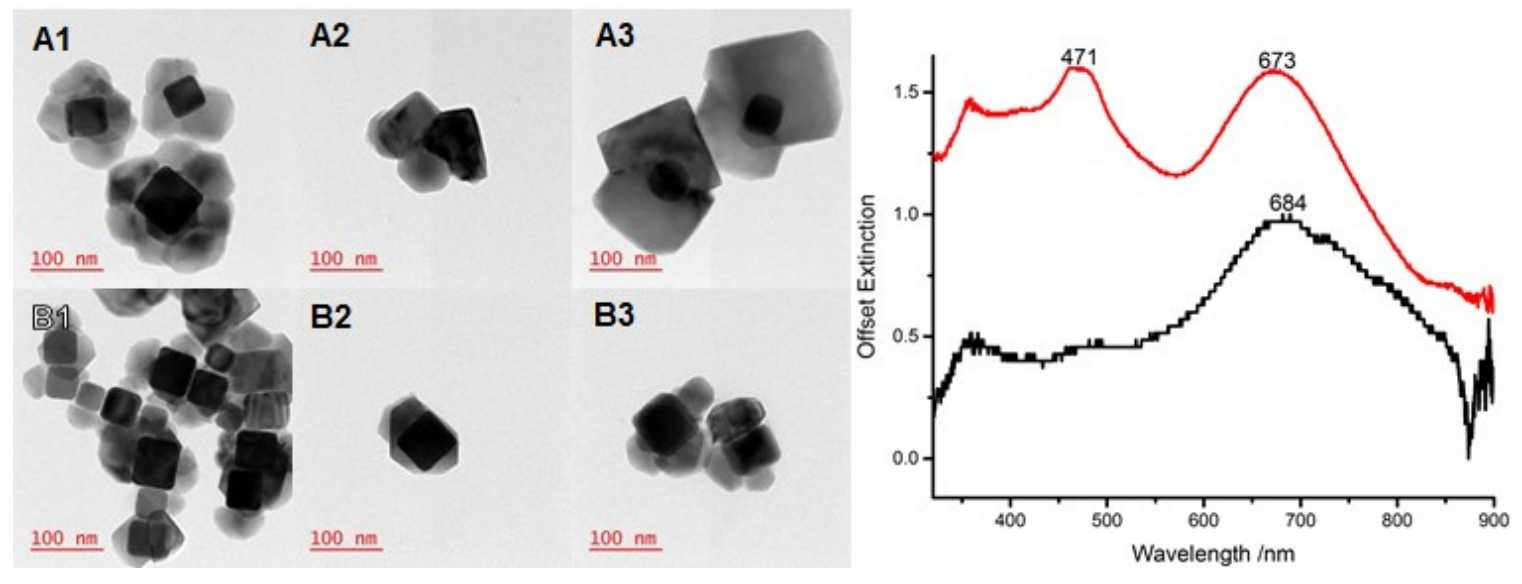

Figure 3.19: Transmission electron micrographs of shells grown with 10 additions of hydrazine, without lactic acid (A1-A3) embedded (slide 13_3) and (B1-B3) supported (slide 11_2) samples. 
(Right) In-solution spectra of samples prepared form (Red) embedded (slide 13_3), (Black) supported (slide 11_2) samples.

\subsection{5 - Slow Addition of Copper, without Lactic Acid}

Shell synthesises were carried out whereby aliquots of copper nitrate solution were added throughout the reaction, every $5 \mathrm{~min}, 10$ times. UV-Vis spectra of the samples after indicated the growth of shells, with new modes present at $660 \mathrm{~nm}$ for the embedded particles (Figure 3.20 A) and $656 \mathrm{~nm}$ for supported particles (Figure $3.20 \mathrm{~B}$ ).
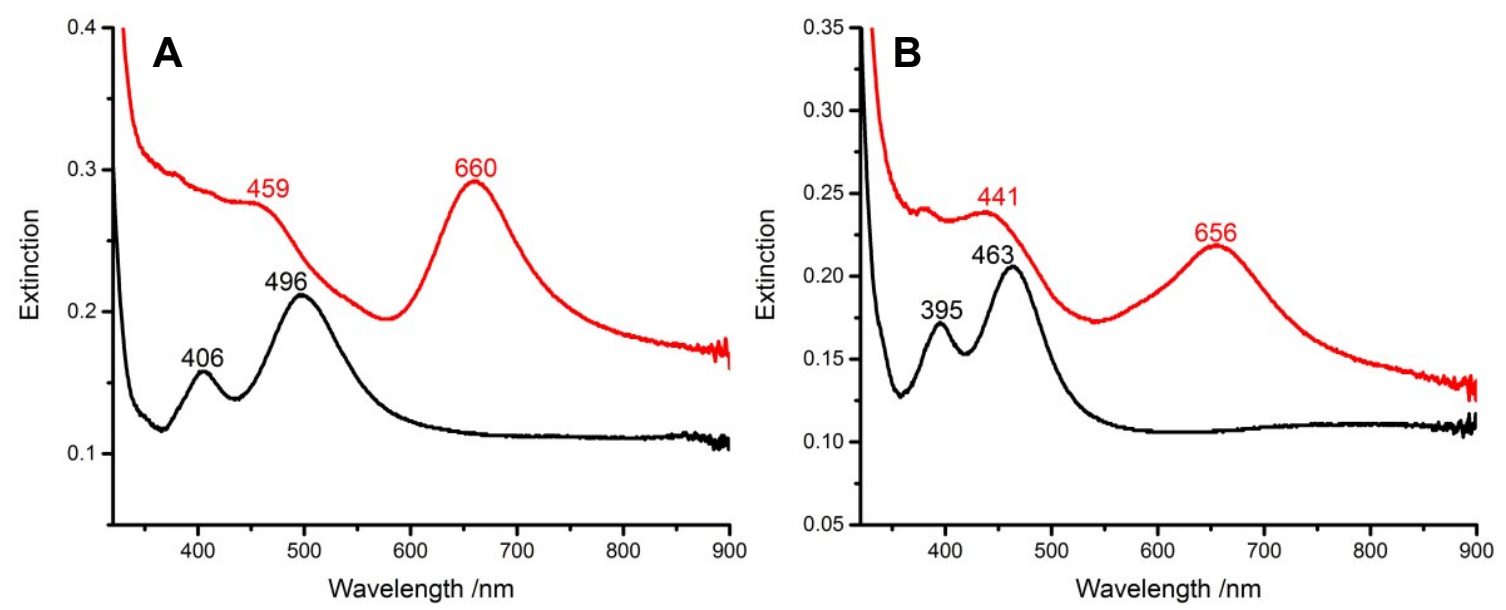

Figure 3.20: Silver nanocube monolayers before (Black) and after copper oxide growth (Red) for (A) embedded (slide 13_1) and (B) supported (slide 11_1) samples.

Subsequently, these samples were removed and characterized with TEM and UV-

Vis. From the TEM images it was observed that the shell was composed of many portions which appear to have nucleated separately and grown together to form the shell for the samples prepared from cubes that were embedded (Figure 3.21 A1-A3) and supported (Figure 3.21 B1-B3). Some of the shell components are roughly cubic in shape; however, many do not have a well-defined structure. The thicknesses, determined with ImageJ, were $38 \mathrm{~nm} \pm 13 \mathrm{~nm}$ and $39 \mathrm{~nm} \pm 10 \mathrm{~nm}$ for those prepared from embedded and supported samples, respectively, indicating a consistent thickness of copper oxide was produced between samples. 
UV-Vis spectra of the samples after removal show hybridization for both the samples which were embedded during growth (Figure 3.21, right, red) and supported during growth (Figure 3.21, right, black). The peaks are located at $678 \mathrm{~nm}$ and $466 \mathrm{~nm}$ (Figure 3.21, right, red) and at $689 \mathrm{~nm}$ and 463 (Figure 3.21, right, black), for the particles prepared from embedded and supported samples, respectively. These samples have similar hybridization.
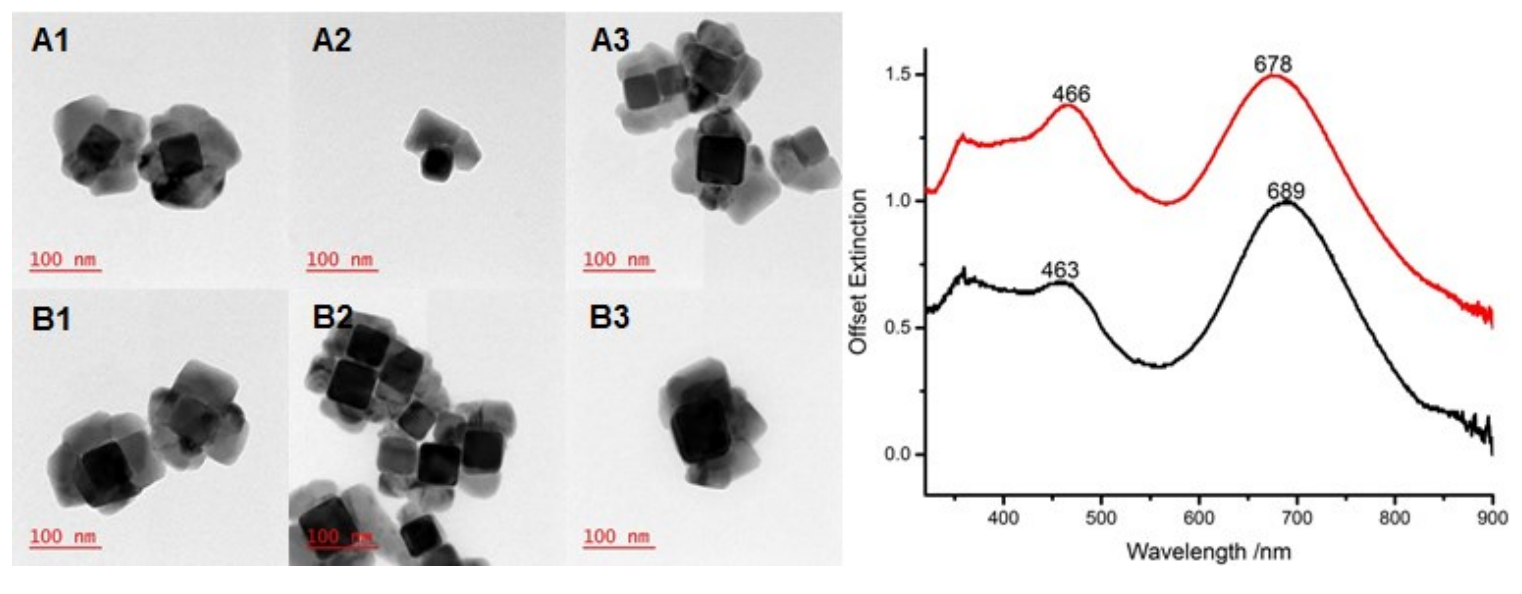

Figure 3.21: (Left) Transmission electron micrographs of shells grown with 10 additions of copper, without lactic acid from (A1-A3) embedded (slide 13_1) and (B1-B3) supported (slide 11_1) samples, (Right) In-solution spectra of samples prepared from (Red) embedded (slide 13_1), (Black) supported (slide 11_1) samples.

\subsection{6 - Concluding Statements on the Puck}

Similarly to the Mechanical Stirrer, the Puck also showed encouraging initial results as a set-up to produce partial shells on the surface of silver nanocubes. In particular, in the presence of lactic acid, it was observed that the produced structures were similar for both the slow addition of hydrazine and the slow addition of copper precursor. Specifically, the produced particles appeared to be composed of structures which resembled 'petals' of $\mathrm{Cu}_{2} \mathrm{O}$ which had grown off of the silver nanocube. For these samples, it was observed that the copper oxide shells grown on the partially embedded 
samples had some hybridization; however, for the samples that were supported during the growth copper oxide, the spectra appeared to resemble very red-shifted cube spectra.

In the absence of lactic acid, the produced structures also appeared to have nucleated at many points on the particle, for both the slow addition of hydrazine and the slow addition of copper precursor. For these samples, some of the produced structures were roughly cubic in shape; however, many had no distinct shape. For the sample to which hydrazine was added slowly, hybridization is observed in solution for the copper oxide grown on partially embedded sample, whereas the sample which was prepared with supported nanocubes, does not display hybridization in solution, and resembles a redshifted cube spectrum. Contrarily, the sample which was prepared with the slow addition of copper precursor, shows in solution hybridization for both the samples prepared from embedded and supported silver nanocube monolayers.

The use of the Puck to prepare core-partial shell nanoparticles was successful. The substrate can be very stably attached to the puck, since it rests on its surface, minimizing the risk of the substrate falling off during the synthesis. In addition, the stirring speed of this set-up could be modified by changing the setting on the stirring hot plate. However, further improvements can be made to this set-up. Specifically, although the stirring rate of this set-up could be modified, high stirring speeds could not be reached with this set-up, and so it was desired to produce a set-up with which the stirring rate could be modified over a larger range, especially faster speeds. In addition, with this setup, if the puck was off-centre in the beaker, it would shake back and forth, no longer smoothly stirring the solution. As such, the Teflon piece was developed in an attempt to further improve the production of these core-partial shell particles. 


\subsection{1 - Teflon Piece}

An initial investigation of the changes in UV-Vis spectra for the growth of copper oxide shells on the surface of embedded silver nanocubes was completed. In particular, these samples were prepared by adding sodium hydroxide, lactic acid and copper nitrate to the solution initially, and adding aliquots of the reducing agent, hydrazine, throughout the reaction. From this, the goal was to determine the changes that occur to the spectra during the shell growth. Hydrazine was added every $5 \mathrm{~min}$, for four sets of substrates, with four, six, eight and ten additions.

After depositing these samples on polystyrene with the Langmuir-Blodgett method, hybrid modes are observed in the spectrum (Figure 3.22, black), with a D mode at $453 \mathrm{~nm}$ and a Q mode at $395 \mathrm{~nm}$. After embedding the samples, the D mode and the Q mode have shifted to $488 \mathrm{~nm}$ and $405 \mathrm{~nm}$, respectively (Figure 3.22, red). With four (Figure 3.22, blue) and six (Figure 3.22, pink) additions of hydrazine, the spectra remain fairly consistent; however, by eight additions of hydrazine (Figure 3.22, green) a new mode has emerged at $586 \mathrm{~nm}$. By ten additions of hydrazine (Figure 3.22, dark blue), this mode has shifted to $604 \mathrm{~nm}$.

For these samples, the expected spectral changes through the growth would first involve the loss of the Q mode as the cubes' surroundings approach having the same affective refractive index for the substrate and superstrate. Subsequently, with the continued growth of copper oxide, a new mode should appear, red-shifted of the initial D mode. This should result because, as the copper oxide shell increases in thickness, the effective refractive index increases, and once it is higher than the polystyrene substrate, 
the $\mathrm{D}$ mode will then be directed into the copper oxide and the $\mathrm{Q}$ mode will be directed into the polystyrene substrate.

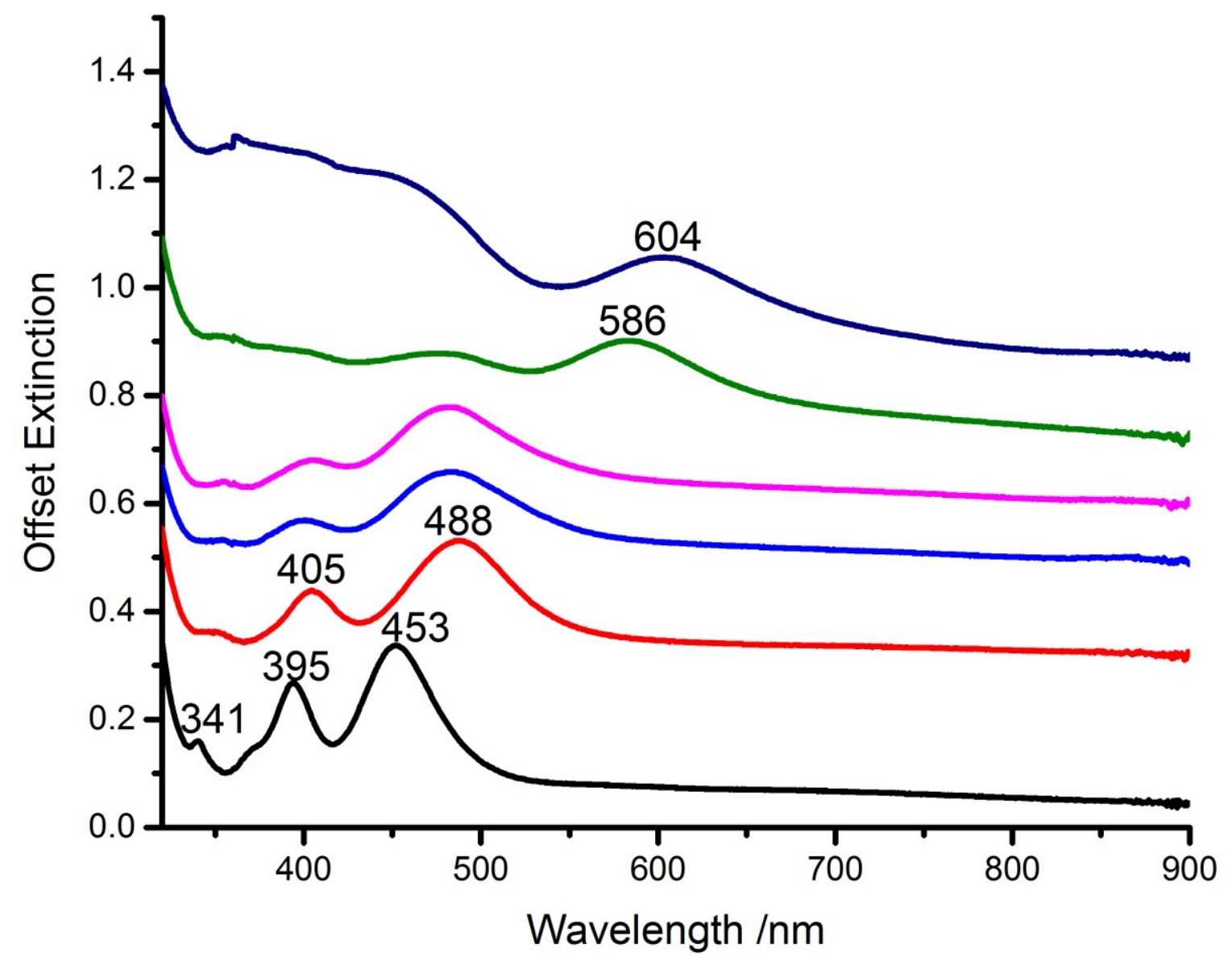

Figure 3.22: UV-Vis spectra of silver nanocube samples initially (Black), embedded into polystyrene (Red) and throughout growth of copper oxide shells, with four additions of hydrazine (Blue), six additions of hydrazine (Pink), eight additions of hydrazine (Green) and ten additions of hydrazine (Dark Blue).

Samples of copper oxide were grown in varying conditions, namely, with additions of copper nitrate or additions of hydrazine, with and without lactic acid, and on samples which were embedded in polystyrene or supported on polystyrene. For all samples, sodium hydroxide was added to the solution first, followed by the other reagents. For the samples where hydrazine was added over many additions, following the addition of sodium hydroxide would be lactic acid (if using), copper nitrate, and finally hydrazine added throughout the reaction. For samples to which hydrazine was added 
(with lactic acid) the solution initially turned blue by $\sim 5 \mathrm{~min}$, following the addition of hydrazine, and subsequently turned green in colour by $\sim 17 \mathrm{~min}$ and finally brown-yellow. For samples to which hydrazine was added throughout the synthesis (without lactic acid), the solution turned blue immediately following the addition of copper nitrate, and then as the reaction proceeded, green by $\sim 8 \mathrm{~min}$, yellow by $\sim 25 \mathrm{~min}$, and brown by $\sim 39 \mathrm{~min}$. The sequence of colour changes of the solution was similar to what occurred under the same

conditions for the 'Mechanical Stirrer'. Again, the blue colour ${ }^{35,68,73,87}$ can be attributed to formation of $\mathrm{Cu}(\mathrm{OH})_{2}$ or $\mathrm{Cu}(\mathrm{OH})_{4}{ }^{2-}$, and the yellow ${ }^{35,87}$ and brown $^{73}$ colours to formation of $\mathrm{Cu}_{2} \mathrm{O}$, or $\mathrm{CuO}$, respectively.

Contrarily, for samples to which copper nitrate was added throughout the synthesis, it was observed that with lactic acid the solution turned yellow within $\sim 1 \mathrm{~min}$, and in the absence of lactic acid turned yellow within $\sim 2-3 \mathrm{~min}$. In both cases, as the reaction progressed, the yellow colour intensified and turned orange over time. For these samples, since the solution contains a higher concentration of reducing agent, with the addition of copper precursor, copper oxide seems to be produced more quickly, based on the yellow colour observed. ${ }^{35,87}$

\subsection{2 - Slow Addition of Hydrazine, with Lactic Acid}

Samples were prepared to which hydrazine was added throughout the synthesis (in the presence of lactic acid) every $5 \mathrm{~min}, 10$ times. The UV-Vis spectra of the substrates, for both embedded (Figure $3.23 \mathrm{~A}$ ) and supported (Figure $3.23 \mathrm{~B}$ ) silver nanocube monolayers, the spectra indicated the growth of copper oxide (red spectra), with the emergence of peaks red-shifted of the initial dipole peak, at $599 \mathrm{~nm}$ and $630 \mathrm{~nm}$ for embedded and supported nanocubes, respectively. 

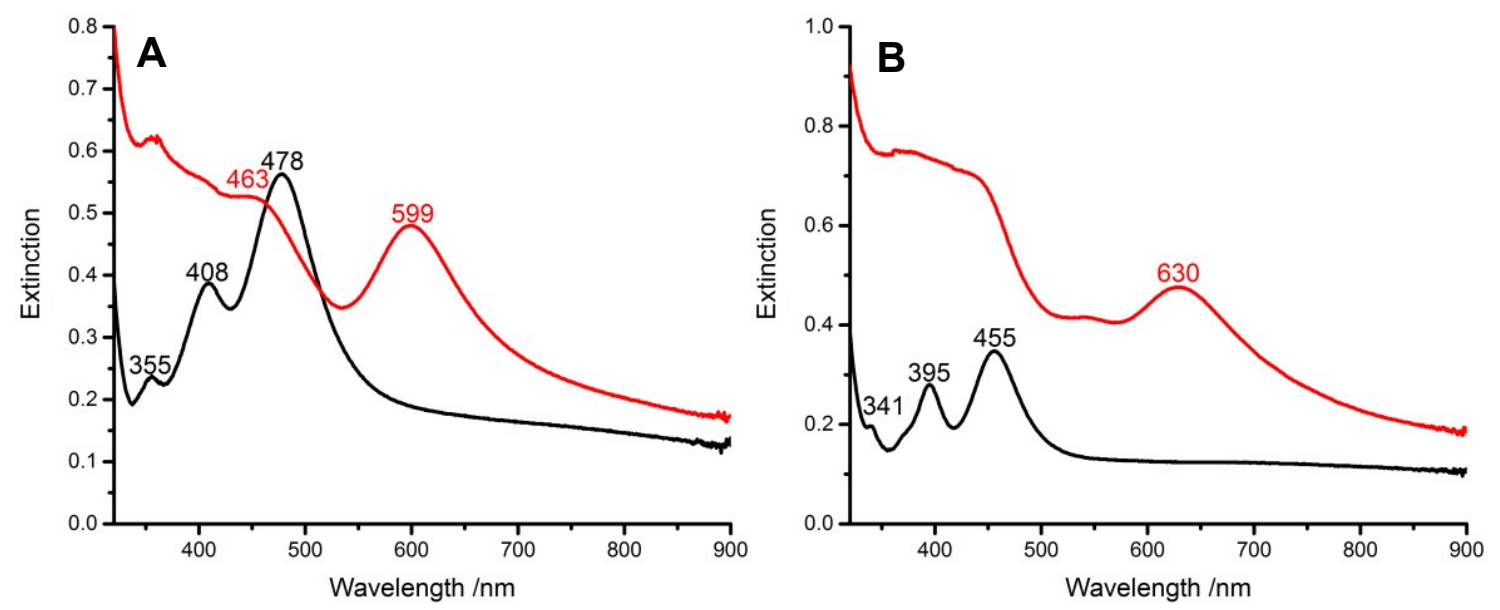

Figure 3.23: Silver nanocube monolayers before (Black) and after copper oxide growth (Red) for (A) embedded (slide 16_4) and (B) supported (slide 1_1) samples.

These particles were then removed from the substrates to determine the resulting morphologies with TEM and to observe their in-solution UV-Vis spectra. In particular, in the images of the sample which was partially embedded, the partial shell morphology can be observed, with exposed part of the silver nanocube visible (Figure 3.24 A1-A3). The shell morphology is similar for both samples that were embedded (Figure 3.24 A1-A3) and for those that were supported (Figure 3.24 B1-B3). The shell structure appears to be composed of many small grains, which had come together resulting in a porous, disorganized shell. The thicknesses of the produced shells were $39 \mathrm{~nm} \pm 20 \mathrm{~nm}$ and 55 $\mathrm{nm} \pm 19 \mathrm{~nm}$ for shells grown on embedded and supported samples, respectively, determined with ImageJ data processing software. These results indicate that the produced shells were of comparable thicknesses; however, the large uncertainty on the thicknesses is the result of the inconsistent shell morphologies produced.

In the UV-Vis spectra for both samples, hybridization of the modes is observed in solution, with D and Q modes at $630 \mathrm{~nm}$ and $464 \mathrm{~nm}$, and $683 \mathrm{~nm}$ and $460 \mathrm{~nm}$, for the samples which were embedded and supported, respectively. In particular, the observed hybridization is better for the samples prepared from the supported silver nanocubes. 

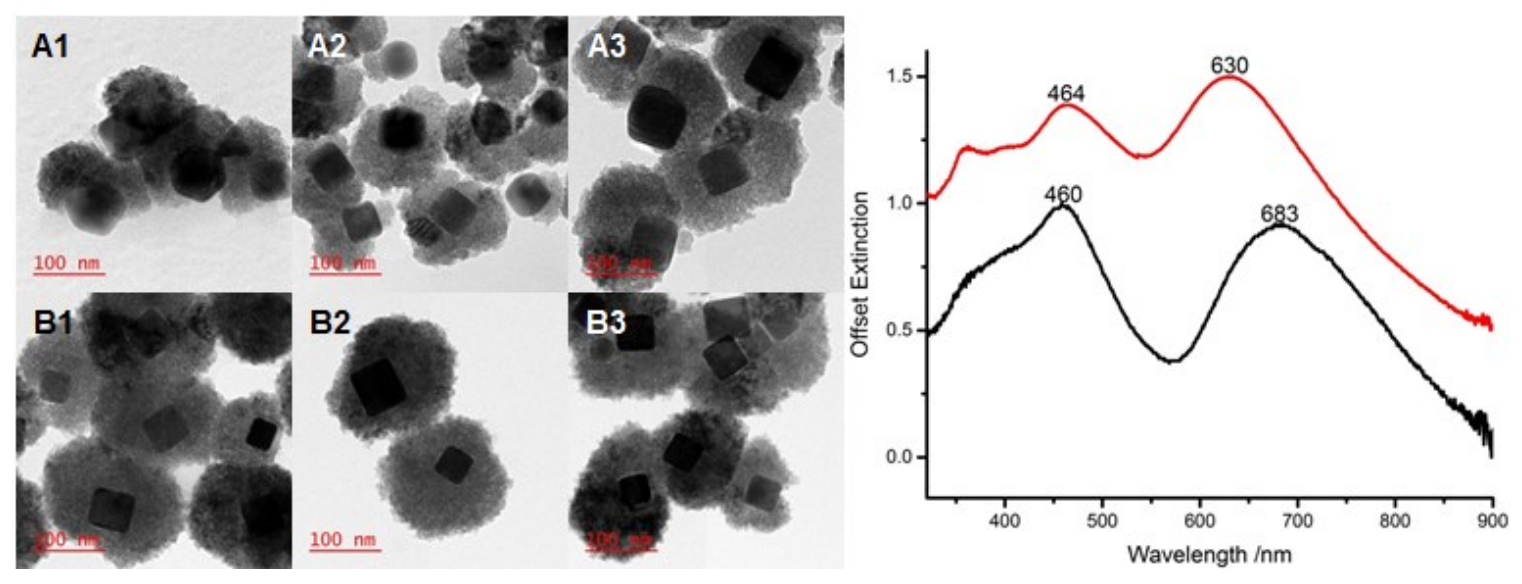

Figure 3.24: (Left) Transmission electron micrographs of shells grown with 10 additions of hydrazine (A1-A3) embedded (slide 16-4) and (B1-B3) supported (slide 1_1) samples. (Right) In-solution spectra of samples prepared from (Red) embedded (slide 16_4) and (Black) supported (slide 1_1) samples.

\subsection{3 - Slow Addition of Copper, with Lactic Acid}

Samples were prepared by adding hydrazine to the solution initially, and adding aliquots of a solution of copper nitrate and lactic acid throughout the reaction (10 additions, one every $5 \mathrm{~min}$ ), for embedded and supported silver nanocube samples. The $\mathrm{UV}-\mathrm{Vis}$ spectra of the slides before and after the synthesis did not indicate that much growth of copper oxide had occurred on the surface of the embedded (Figure 3.25 A) or supported (Figure 3.25 B) nanocubes. For the embedded silver nanocubes (Figure 3.25 A), the D and Q modes are still observable, and have blue-shifted slightly, which could indicate some rounding of the sample had occurred. For the supported samples (Figure $3.25 \mathrm{~B}$ ), a change is seen in the UV-Vis spectra whereby a shoulder is observed at $\sim 650$ $\mathrm{nm}$, which suggests some copper oxide growth on the surface of the particles. 

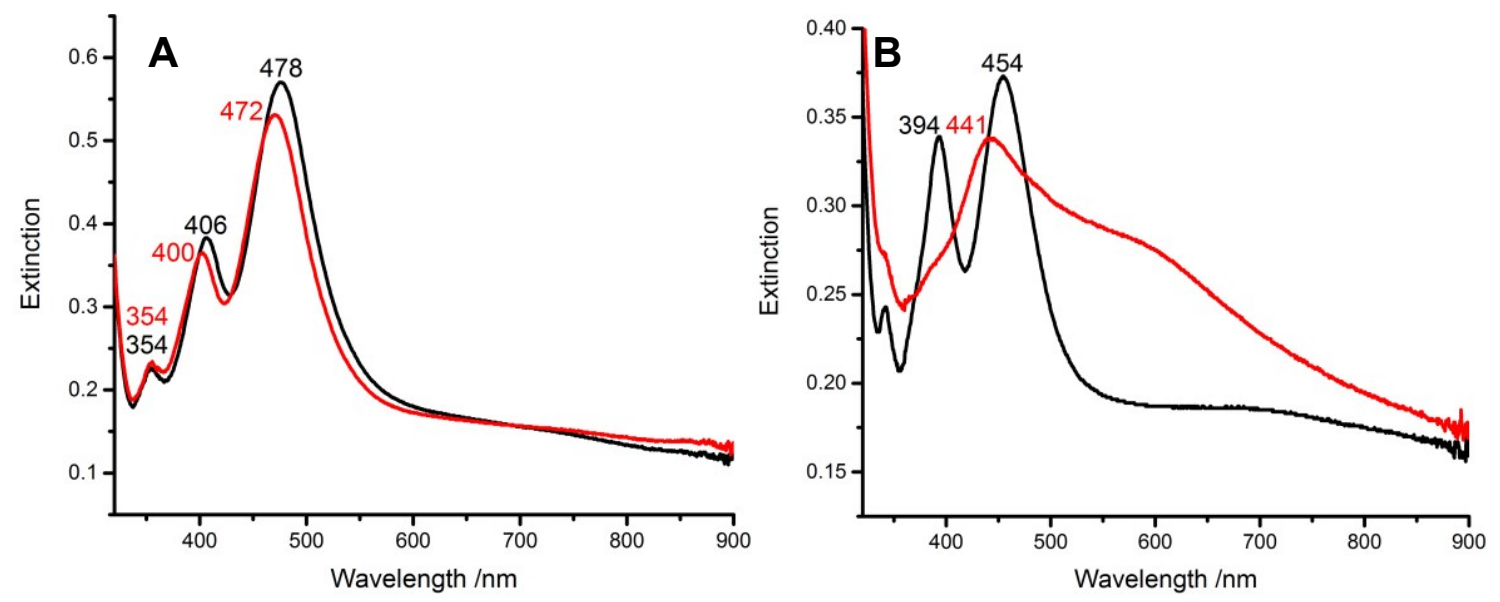

Figure 3.25: Silver nanocube monolayers before (Black) and after copper oxide growth (Red) for (A) embedded (slide 16_2) and (B) supported (slide 22_2) samples

Subsequent removal of the samples and obtainment of TEM images was consistent with the UV-Vis spectra of the samples on the substrates. In particular, very minimal growth is observable on the surface of the particles that were embedded (Figure 3.26 A1-A3). Additionally, some of the cubes are observed to have rounded (Figure 3.26 A1-A3). The corresponding UV-Vis spectra of this sample (Figure 3.26, right, red) is shaped like a spectrum of cubic particles with a main dipole at $492 \mathrm{~nm}$ and a slight peak at $\sim 350 \mathrm{~nm}$, which is consistent with minimal growth on the particles.

For the sample that was supported on the surface of the particles, the results are similar to their embedded counterparts, with not a lot of growth on the surface; however, as more area of the cube was exposed, some growth is observed on a larger area of the nanoparticle surface (Figure $3.26 \mathrm{~B} 1-\mathrm{B} 3$ ). In these samples, patches of growth are observed, indicating that some copper oxide nucleated at different areas on the cube surface and have not grown together into a complete shell (Figure 3.26 B1-B3). The UVVis spectrum of this sample (Figure 3.26, right, black) shows further red-shift of the peak to $587 \mathrm{~nm}$, with the presence of a shoulder at $\sim 450 \mathrm{~nm}$, indicating that hybridization of the modes has started, however, not enough to separate the peaks well. 
From these results, it is observed that the growth on the nanoparticles is poor, which is expected to be due to the Teflon piece causing a lot of bubbles. Since the synthesis was carried out under air, oxygen would have been mixed into the solution from the bubbles. Since the hydrazine was added all at the beginning of the reaction, it seems that the lack of growth is due to hydrazine reacting with oxygen, ${ }^{119}$ which competed with the reduction of the copper precursor to copper (I) oxide. Similarly, $\mathrm{Cu}^{2+}$ can be reformed from $\mathrm{Cu}^{+}$in the presence of oxygen, ${ }^{119}$ which could also reduce the growth of the copper oxide. In particular, the thicknesses of the patches were measured with ImageJ software and determined to be $14 \mathrm{~nm} \pm 7 \mathrm{~nm}$ and $16 \mathrm{~nm} \pm 6 \mathrm{~nm}$ at the thickest portion of copper oxide shell, for those grown on embedded and supported samples, respectively, which are much thinner than the shells produced under other conditions. As such, under these conditions, the 'Teflon Piece' set-up is not appropriate for the growth of copper oxide shells on the surface of silver nanoparticles.
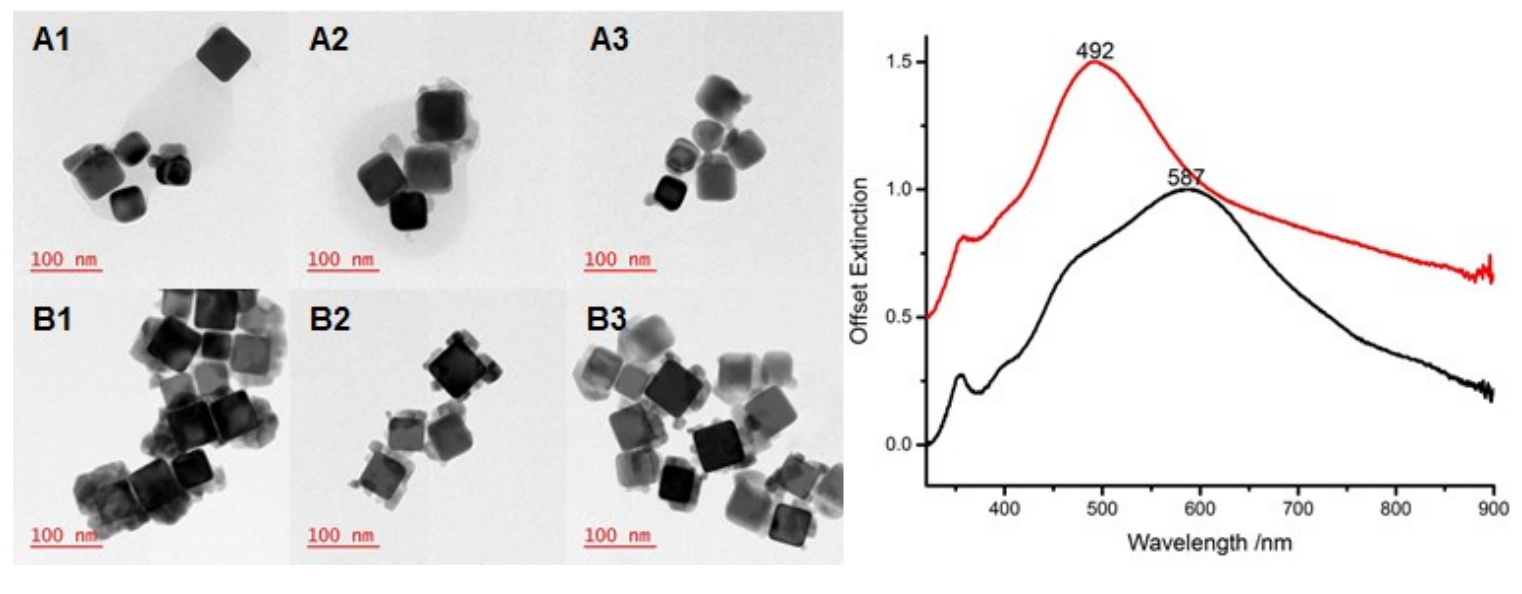

Figure 3.26: (Left) Transmission electron micrographs of shells grown with 10 additions of copper, with lactic acid (A1-A3) embedded (slide 16_2) and (B1-B3) supported (slide 22_2) samples. (Right) In-solution spectra of samples prepared from (Red) embedded (slide 16_2), (Black) supported (slide 22_2) samples. 


\subsection{4 - Slow Addition of Hydrazine, without Lactic Acid}

Samples were also prepared using the 'Teflon Piece' by adding hydrazine throughout the reaction (one addition every $5 \mathrm{~min}, 10$ times), without lactic acid. For these samples, the UV-Vis spectra indicate the growth of the copper oxide shells, from the emergence of a peak red-shifted of the initial dipole peaks, at $605 \mathrm{~nm}$ and $612 \mathrm{~nm}$ for the embedded (Figure 3.27 A) and supported (Figure 3.27 B) samples, respectively.
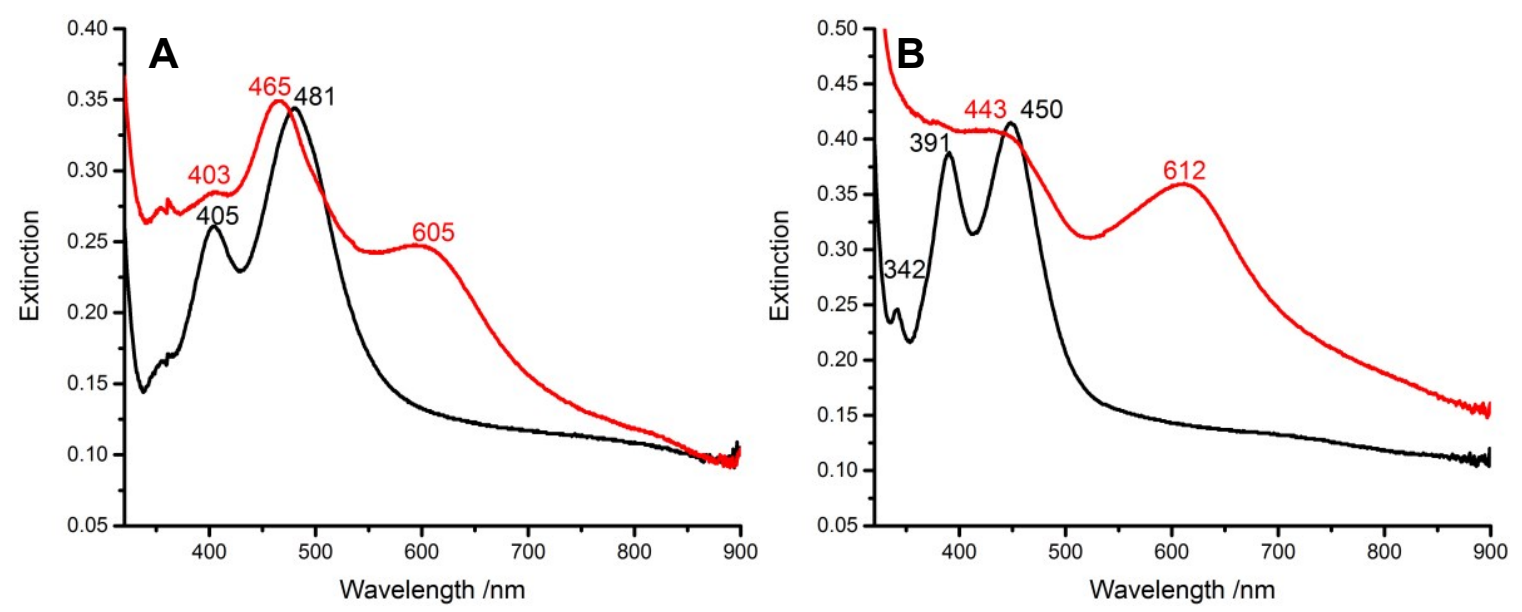

Figure 3.27: Silver nanocube monolayers before (Black) and after copper oxide growth (Red) for (A) embedded (slide 16_3) and (B) supported (slide 18_2) samples.

Thereafter, the samples are removed from the substrates and re-suspended in solution. TEM images are obtained for the embedded (Figure 3.28 A1-A3) and supported samples (Figure 3.28 B1-B3) samples. For these particles, the resulting copper oxide structures are observed to be mostly cubic in structure, which could have resulted due to the fact that the lowest energy facet for $\mathrm{Cu}_{2} \mathrm{O}$ is the $\{100\}$ facet, ${ }^{70}$ so it was most stable, especially in the absence of other structural guiding agents, such as lactate. It can be observed in these samples that, on many of the particles, the shells nucleated at many different parts of the particle and grew into each other (Figure 3.28 A1-A3, B1-B3). These results are similar to the morphology of the shell obtained under the same conditions using the Mechanical Stirrer (Figure 3.11). The thicknesses of the produced 
shells were $36 \mathrm{~nm} \pm 17 \mathrm{~nm}$ and $36 \mathrm{~nm} \pm 10 \mathrm{~nm}$ for samples prepared from embedded and supported nanocubes, respectively, determined with ImageJ data processing software, indicating consistent produced thicknesses.

In the UV-Vis spectra hybridization is observed for the particles that were embedded with the D and Q modes being located at $637 \mathrm{~nm}$ and $480 \mathrm{~nm}$, respectively. The spectra for the samples prepared from supported silver nanocube monolayers resemble a red-shifted cube spectrum, with a main peak at $663 \mathrm{~nm}$ another at $461 \mathrm{~nm}$.
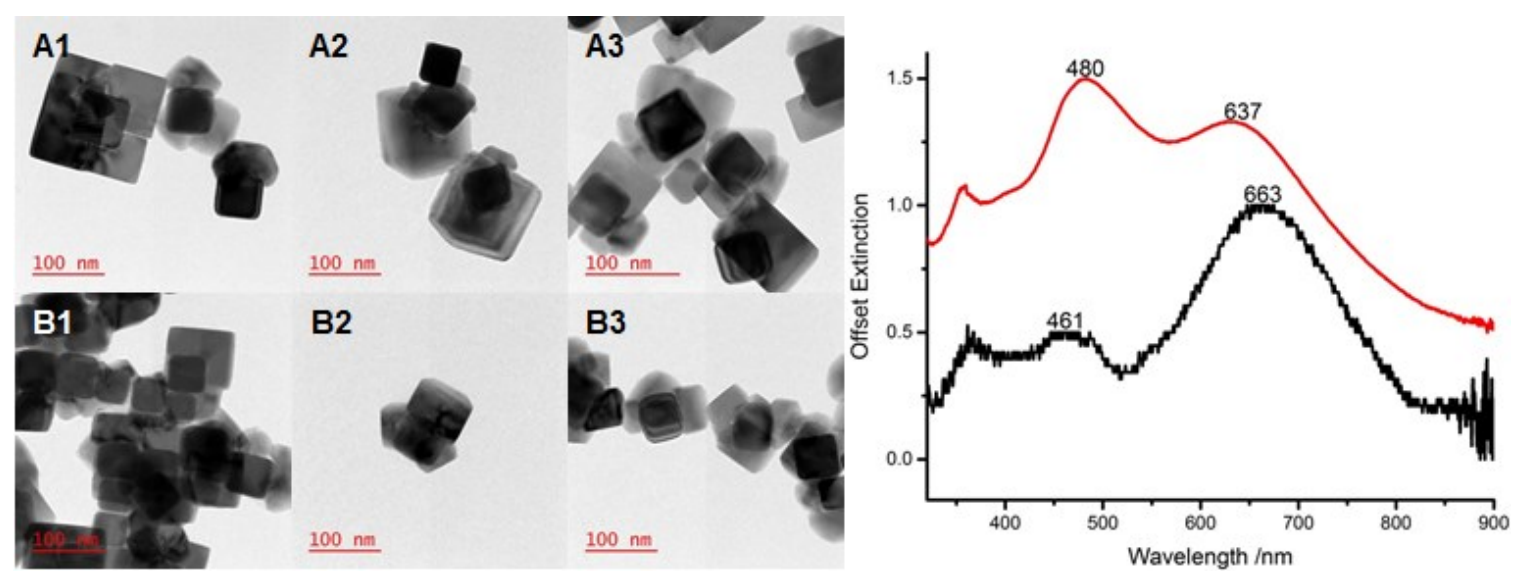

Figure 3.28: (Left) Transmission electron micrographs of shells grown with 10 additions of hydrazine, without lactic acid from (A1-A3) embedded (slide 16_3) and (B1-B3) supported (slide 18_2) samples, (Right) In-solution spectra of samples prepared from (Red) embedded (slide 16_3), (Black) supported (slide 18_2) samples.

\subsection{5 - Slow Addition of Copper, without Lactic Acid}

Furthermore, samples were prepared by adding portions of a solution of copper nitrate (without lactic acid) throughout the reaction (added every 5 min, 10 times). The UV-Vis spectra of these samples on the substrates indicated growth, with peaks emerging at $670 \mathrm{~nm}$ and $677 \mathrm{~nm}$, for embedded (Figure $3.29 \mathrm{~A}$ ) and supported (Figure $3.29 \mathrm{~B}$ ) nanoparticles, respectively. 

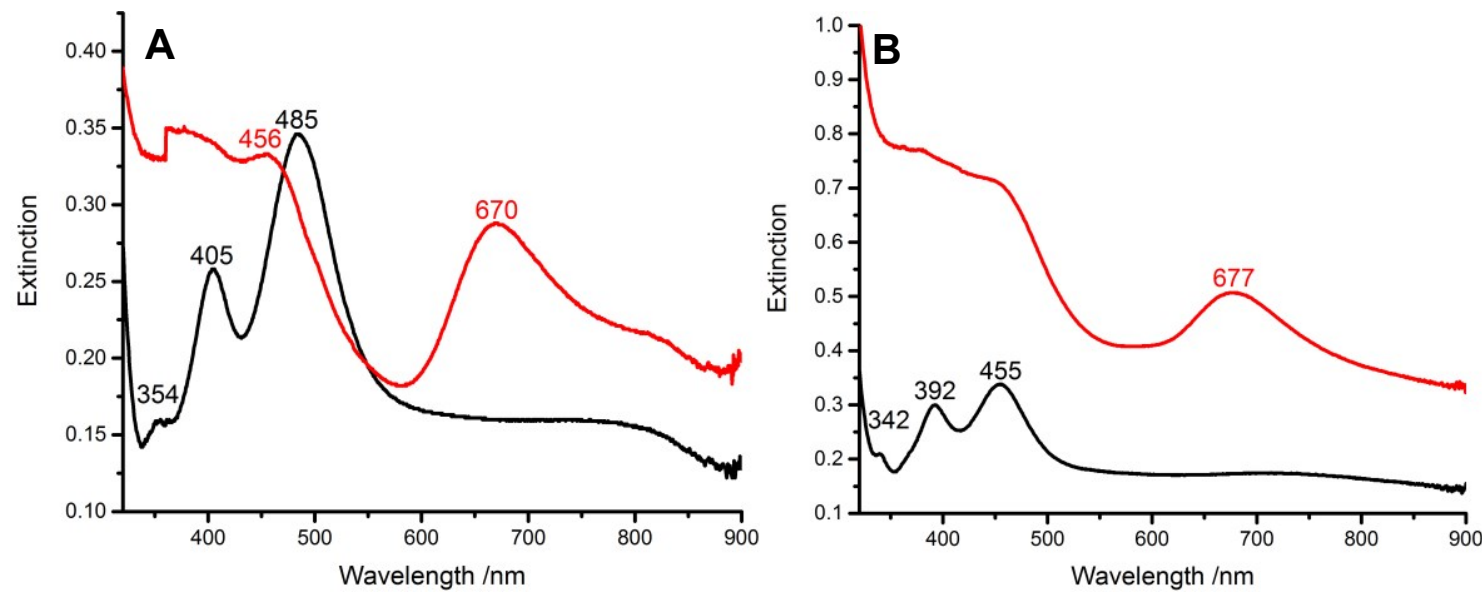

Figure 3.29: Silver nanocube monolayers before (Black) and after copper oxide growth (Red) for (A) embedded (slide 16_1) and (B) supported (slide 18_1) samples.

Moreover, upon removal of these samples, TEMs were obtained for structures prepared from the embedded (Figure 3.30 A1-A3) and supported (Figure 3.30 B1-B3) nanocubes. The shell is perceived to be composed of the growth of many portions which had nucleated and grown together, forming the shell. In addition, many sections of the shell appear to be semi-cubic in structure; however, these are not as defined as the cubic shapes as were obtained under the conditions where hydrazine was added throughout the reaction (Figure 3.30 A1-A3, B1-B3), instead of copper precursor. The thicknesses produced under these conditions were determined to be $42 \mathrm{~nm} \pm 17 \mathrm{~nm}$ and $43 \mathrm{~nm} \pm 14$ $\mathrm{nm}$ for those grown on embedded and supported samples, respectively, with the use of ImageJ data processing software, indicating consistent produced thicknesses.

The UV-Vis spectra of the samples prepared were obtained for the particles in solution and it was observed that there was hybridization of the D and Q modes in solution; for the initially embedded sample (Figure 3.30, right, red) and the supported sample (Figure 3.30, left, black), the D and Q modes are observed at $692 \mathrm{~nm}$ and $470 \mathrm{~nm}$, and 718 and $472 \mathrm{~nm}$, respectively. 

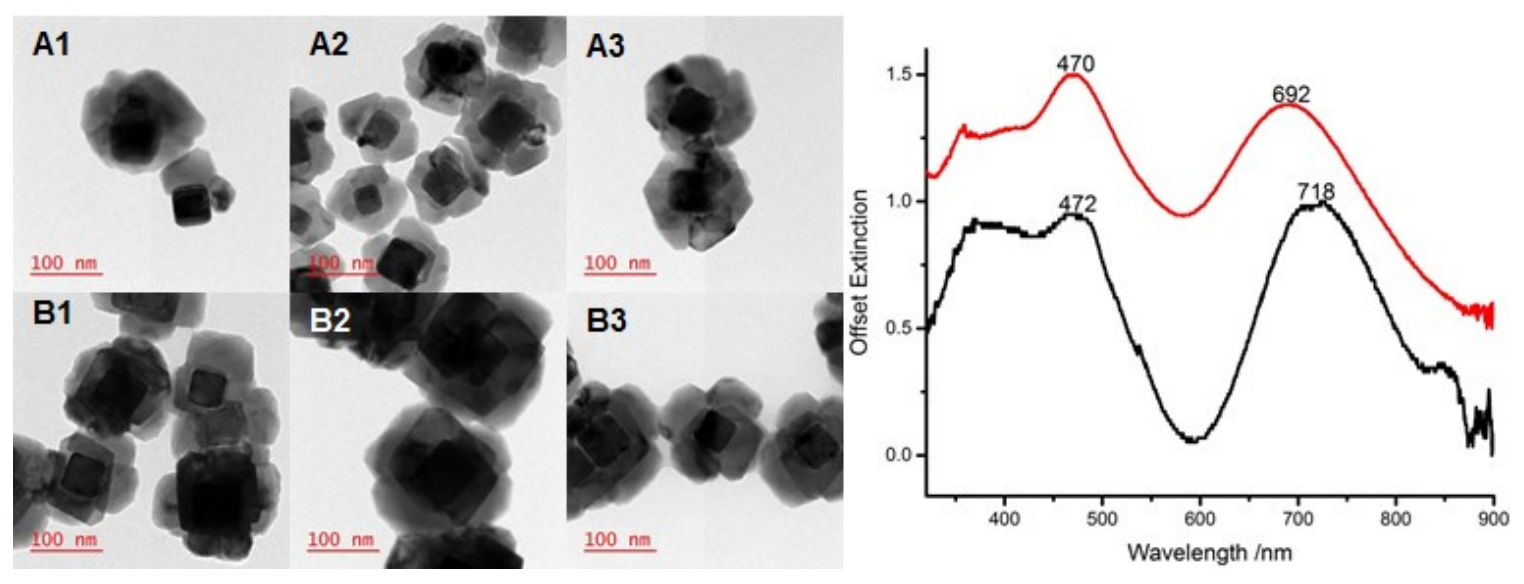

Figure 3.30: (Left) Transmission electron micrographs of shells grown with 10 additions of copper, without lactic acid from (A1-A3) embedded (slide 16_1) and (B1-B3) supported (slide 18_1) samples, (Right) In-solution spectra of samples prepared from (Red) embedded (slide 16_1), (Black) supported (slide 18_1) samples.

\subsection{6 - Concluding Statements on the Teflon Piece}

The 'Teflon Piece' was successful for the preparation of core-partial shell silver copper oxide nanoparticles, under certain conditions. In particular, for samples that were prepared in the presence of lactic acid with the slow addition of hydrazine, the resulting shell structure was porous. Both the particles produced from embedded and supported silver nanocubes had substrate-induced hybridization present in the UV-Vis spectra of the particles in solution. Structures produced with the slow addition of copper, however, had very little growth, which was attributed to the production of a lot of bubbles. This was expected to have resulted in hydrazine reacting with oxygen, ${ }^{119}$ and reducing the growth of copper oxide shells. The shape of the in-solution spectra resembled that of cubes for the particles produced with the partially embedded nanocubes; however, the spectra appears to be starting to split due to hybridization, since there was more overall copper oxide growth on these particles.

For the samples produced without lactic acid, with the slow addition of hydrazine, the produced shell structures were boxes, often with many having nucleated on the 
surface of each particle. In the case of the samples produced without lactic acid, many cubic-shaped copper oxide shell particles were grown when hydrazine was added slowly. Hybridization was observed in the in-solution spectra of the structures prepared from the partially embedded nanocubes, while the in-solution spectra of the structures prepared from the supported nanocubes resemble a red-shifted cube spectrum.

For samples prepared without lactic acid, with the slow addition of the copper precursor, semi-cubic shaped shell patches were produced; however, these were not as sharp as the ones prepared with the slow addition of hydrazine. Both the structures prepared from nanocubes which were embedded and supported showed hybridization of the modes in-solution in the UV-Vis spectra.

This set-up had the advantages of being able to stir at faster rates than the puck, and the speed was adjustable. In addition, the Teflon piece was removable and easy to thoroughly clean. However, this set-up produced a lot of bubbles, which impacted the growth of the copper oxide shells, especially for the case where lactic acid and hydrazine had been added at once and the copper precursor was added slowly, throughout the reaction. As such, a set-up in which the synthesis could be done under nitrogen was designed, in an attempt rectify this issue.

\subsection{1 - New Set-up}

This set-up was used, taking into consideration the lessons learned from the other setups: namely, this set-up allows the reactions to be carried out under nitrogen, has adjustable speeds, and is glass, and thus non-reactive and easy to clean. 
With this set-up, copper oxide was grown on embedded samples, with increasing amounts of $\mathrm{NaOH}$ and $\mathrm{CuNO}_{3}$, with and without lactic acid, keeping the ratio between these reagents consistent, in an attempt to adjust the sizes of produced shells.

\subsection{2 - Hydrazine and Copper Added Quickly, without Lactic Acid}

The first set of samples was prepared in the absence of lactic acid, and, in all cases, $250 \mathrm{~mL}$ of water was used as the solvent and $220 \mu \mathrm{L}$ of $25 \%$ hydrazine was used as the reducing agent, which was added after the addition of $\mathrm{NaOH}$ and $\mathrm{CuNO}_{3}$. The amounts of $5 \mathrm{M} \mathrm{NaOH}$ and $0.5 \mathrm{M} \mathrm{CuNO}_{3}$ added to the samples were each $550 \mu \mathrm{L}, 450$ $\mu \mathrm{L}$ and $350 \mu \mathrm{L}$; the UV-Vis spectra can be seen in Figure $3.31 \mathrm{~A}, \mathrm{~B}, \mathrm{C}$, for these concentrations, respectively. Under these conditions increasing the concentrations of both $\mathrm{NaOH}$ and $\mathrm{CuNO}_{3}$ does not result in a significant increase in the amount of shell growth observable in the UV-Vis spectra (Figure 3.31).

Particularly, as the copper oxide shell first starts to grow on the surface of the nanoparticle, the hybrid Q mode will be affected, and is observed to deplete in the UVVis spectra. As the copper oxide shell continues to grow, a new mode, red-shifted of the hybrid D mode, will begin to appear in the UV-Vis spectra for thick shells. This is not observed in the spectra, except for a slight bump at $\sim 650 \mathrm{~nm}$ in Figure $3.31 \mathrm{~B}$ (corresponding to the intermediate amount of $\mathrm{NaOH}$ and $\mathrm{CuNO}_{3}$ ).

In addition, from the TEMs, the morphologies of the produced copper oxide shells are observed, and it is noted that in all cases the growth is not uniform. The structures produced with the lowest concentrations of $\mathrm{NaOH}$ and $\mathrm{CuNO}_{3}$ have small islands of copper oxide, which seem to grow predominantly off of the corners, with some also growing off of other areas (Figure 3.32 C). The intermediate concentrations of $\mathrm{NaOH}$ and 
$\mathrm{CuNO}_{3}$ produced shells that were more complete than those produced with the lowest concentrations; however, some of the nanoparticles were also uncoated, which could result from these particles being aggregated on the surface of the polystyrene, such that copper oxide cannot grow on their surfaces (Figure 3.32B). Similarly, not much growth is observed on the surface of the nanoparticles with the highest concentrations (Figure 3.32A). The thicknesses of the copper oxide grown was measured with ImageJ software and found to be $15 \mathrm{~nm} \pm 7 \mathrm{~nm}, 21 \mathrm{~nm} \pm 11 \mathrm{~nm}$ and $24 \mathrm{~nm} \pm 12 \mathrm{~nm}$, for the samples prepared with $350 \mu \mathrm{L}, 450 \mu \mathrm{L}$ and $550 \mu \mathrm{L}$ of reagents, respectively. These measurements suggest that as the amount of $\mathrm{NaOH}$ and $\mathrm{CuNO}_{3}$ is increased the produced shells increase in thickness; however, the large uncertainty on the thickness values are due to the inconsistent shell morphology.

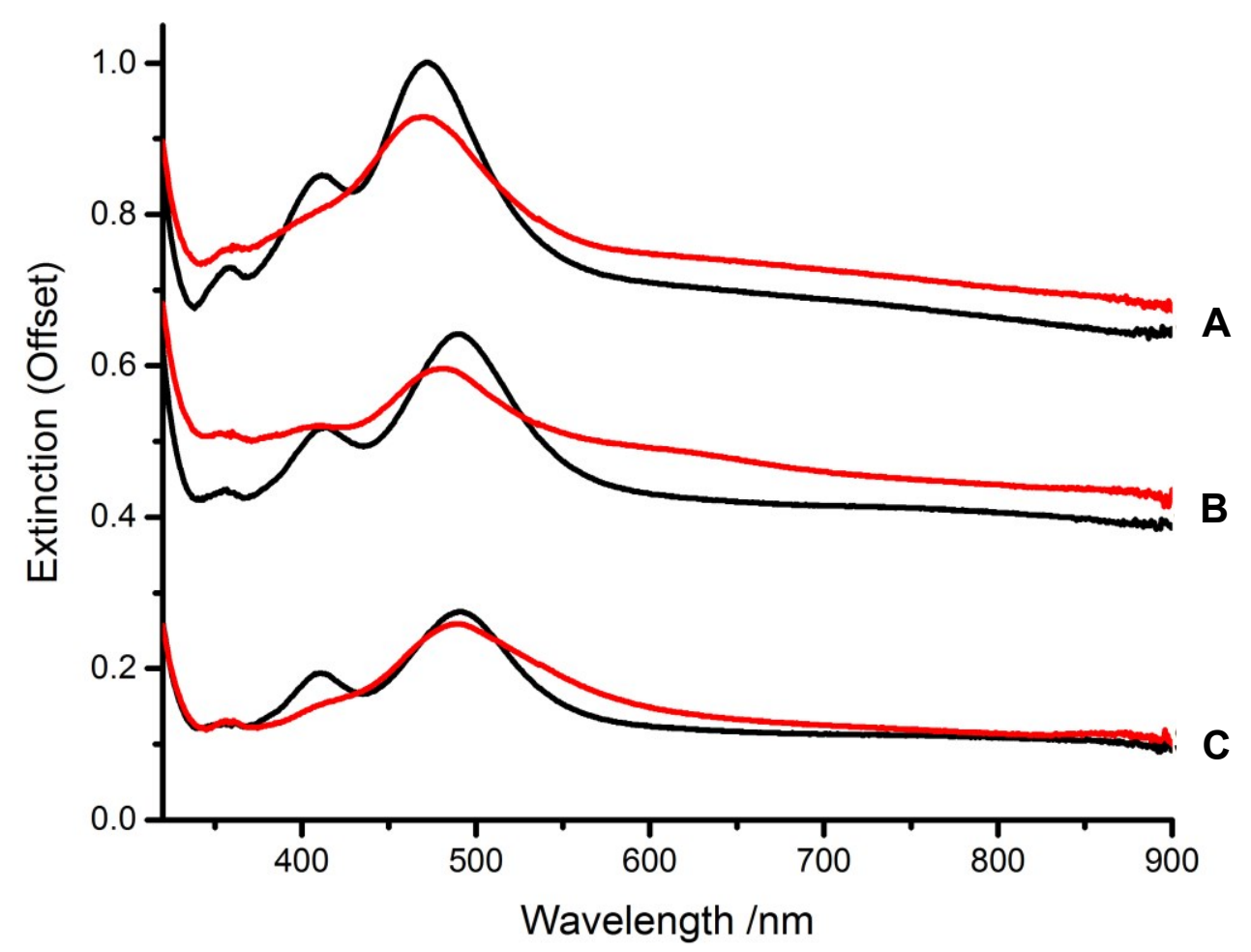

Figure 3.31: UV-Vis spectra of embedded silver nanocube monolayers before (Black) and after copper oxide growth (Red) for different added amounts of $5 \mathrm{M} \mathrm{NaOH}$ and $0.5 \mathrm{M} \mathrm{Cu}\left(\mathrm{NO}_{3}\right)_{2}(\mathrm{~A}) 550 \mu \mathrm{L}$ (slide 23_2), (B) $450 \mu \mathrm{L}$ (slide 5_3), (C) $350 \mu \mathrm{L}$ (slide 5_1). 


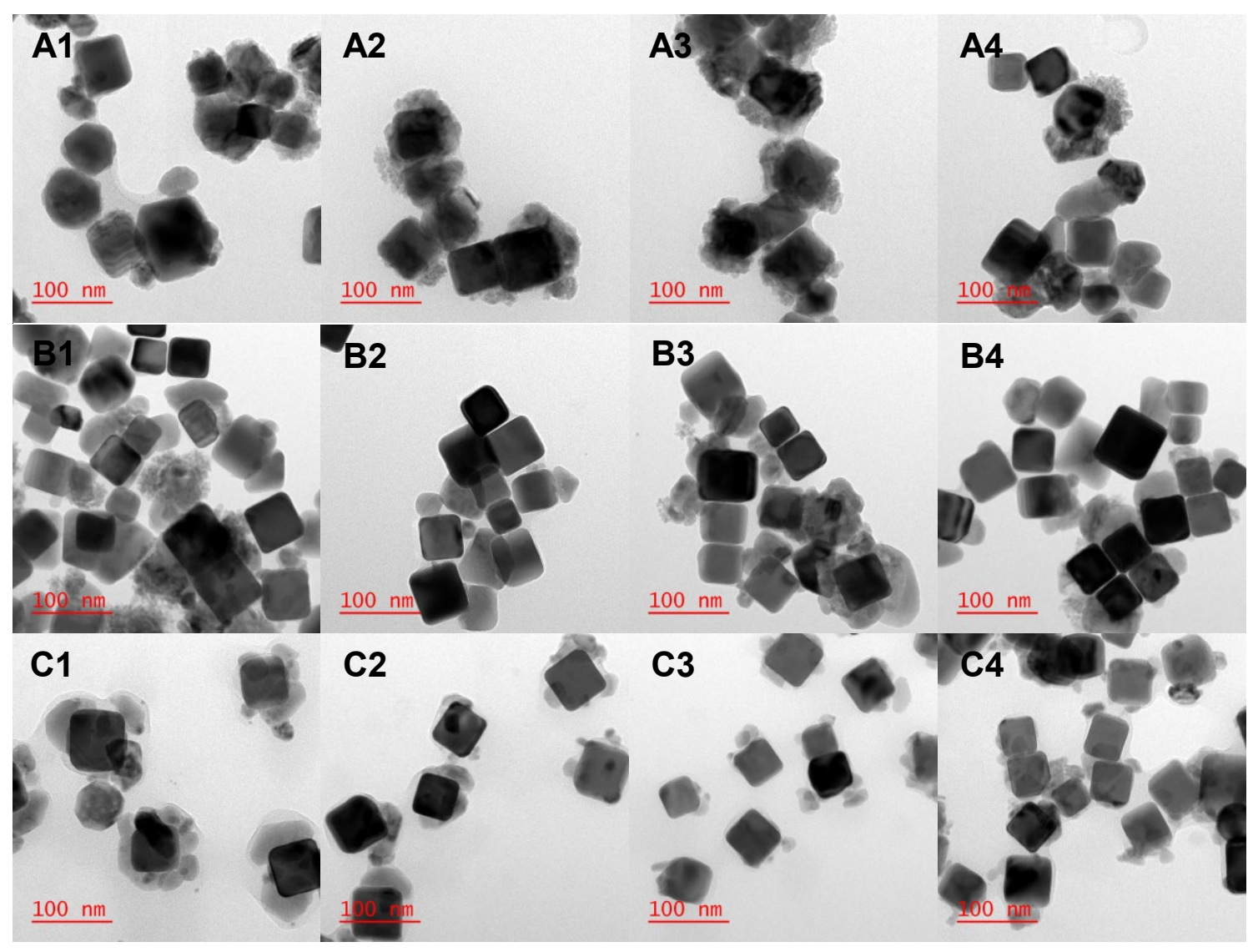

Figure 3.32: TEM images for structures produced from embedded nanocube monolayers with different added amounts of $5 \mathrm{M} \mathrm{NaOH}$ and $0.5 \mathrm{M} \mathrm{Cu}\left(\mathrm{NO}_{3}\right)_{2}$ (A) $550 \mu \mathrm{L}$ (slide 23_2), (B) $450 \mu \mathrm{L}$ (slide 5_3), (C) $350 \mu \mathrm{L}$ (slide 5_1).

\subsection{3 - Hydrazine and Copper Added Quickly, with Lactic Acid}

This experiment was also conducted with the addition of lactic acid. In all cases, $250 \mathrm{~mL}$ of water was again used as the solvent, and $220 \mu \mathrm{L}$ of $25 \%$ hydrazine was used as the reducing agent, which was added after the addition of $\mathrm{NaOH}$, lactic acid and $\mathrm{CuNO}_{3}$. The amounts of $5 \mathrm{M} \mathrm{NaOH}, 4 \mathrm{M}$ lactic acid and $0.5 \mathrm{M} \mathrm{CuNO}_{3}$ added to the samples were each $550 \mu \mathrm{L}, 450 \mu \mathrm{L}$ and $350 \mu \mathrm{L}$; with the UV-Vis spectra seen in Figure $3.34 \mathrm{~A}, \mathrm{~B}, \mathrm{C}$ respectively.

From the UV-Vis spectra of the samples, it can be observed that with the lowest amounts of $\mathrm{NaOH}, \mathrm{CuNO}_{3}$ and lactic acid, the hybrid Q mode is depleted and a peak redshifted of the hybrid D mode is present in the spectra after the growth of copper oxide 
(Figure $3.34 \mathrm{C}$ ). With the intermediate amounts of precursors added, the hybrid Q mode is similarly depleted, and the mode red-shifted of the hybrid D mode is more pronounced, indicating more growth of copper oxide on this sample than the previous one (Figure 3.34 B). However, further increasing the concentration of the precursors does not result in thicker shells. The spectrum of the particles after copper oxide growth with the highest concentration of precursors shows the hybrid Q mode is less defined; however, a peak had not developed red-shifted of the hybrid D mode (Figure 3.34 A). In addition, the substrate was observed to be bright, matte orange in colour and after removal in toluene, the solution similarly was bright orange, indicating the growth of many copper oxide nanoparticles in solution, which had deposited onto the substrate.

Under these conditions, increasing the concentrations from the low (Figure 3.35 A) to intermediate (Figure $3.35 \mathrm{~B}$ ) amount added results in an increased growth of copper oxide, while increasing the concentration to the highest amount (550 $\mu \mathrm{L}$ of each) resulted in growth of copper oxide particles in solution, and as such, less growth of copper oxide shells on the silver nanocubes (Figure 3.35 A).

The TEMs were consistent with the observed spectral changes. Particularly, the sample that was subjected to the highest concentrations of precursors had very little growth on the silver nanocubes. However, many large particles were also observed (Figure $3.35 \mathrm{~A}$ ), indicating that under these conditions, the concentrations were high enough such that homogeneous nucleation of the copper oxide in solution was favourable to the heterogeneous nucleation on the silver nanocube's surface. It is interesting that this result is observed only in the presence of lactic acid (Figure $3.35 \mathrm{~A}$ ), and not in its 
absence (Figure $3.32 \mathrm{~A}$ ), which suggests the presence of lactic acid makes homogeneous nucleation more favourable at these concentrations.

Additionally, the low and intermediate amounts of precursors (Figure 3.35 B,C) resulted in more growth on the surface than in the same conditions without lactic acid (Figure $3.32 \mathrm{~B}, \mathrm{C}$ ), again indicating that lactic acid facilitates the growth of copper oxide, under these conditions. Both the low and intermediate amount of precursor with lactic acid resulted in the growth of shells that seem to be the result of nucleation at many points on the nanoparticle's surface further growing together to form the shell (Figure 3.35 B, C). Increasing the concentration of precursors from the low ( $350 \mu \mathrm{L}$ of each) to the intermediate $(450 \mu \mathrm{L}$ of each) resulted in increased shell growth, with the intermediate concentration having fewer gaps between portions of the shell that have grown together (Figure $3.35 \mathrm{~B}, \mathrm{C}$ ). Specifically, the thicknesses of the shells were measured with ImageJ software, and determined to be $21 \mathrm{~nm} \pm 7 \mathrm{~nm}$ for those produced with $350 \mu \mathrm{L}$ of precursor and $22 \mathrm{~nm} \pm 7 \mathrm{~nm}$ with the addition of $450 \mu \mathrm{L}$ of precursor. Although the thickness produced is very similar between the low and intermediate amounts, the shells have fewer gaps and are more complete with the intermediate amounts of precursor. With the addition of $550 \mu \mathrm{L}$ of reagents the produced copper oxide particles had diameters of $167 \mathrm{~nm} \pm 30 \mathrm{~nm}$. 


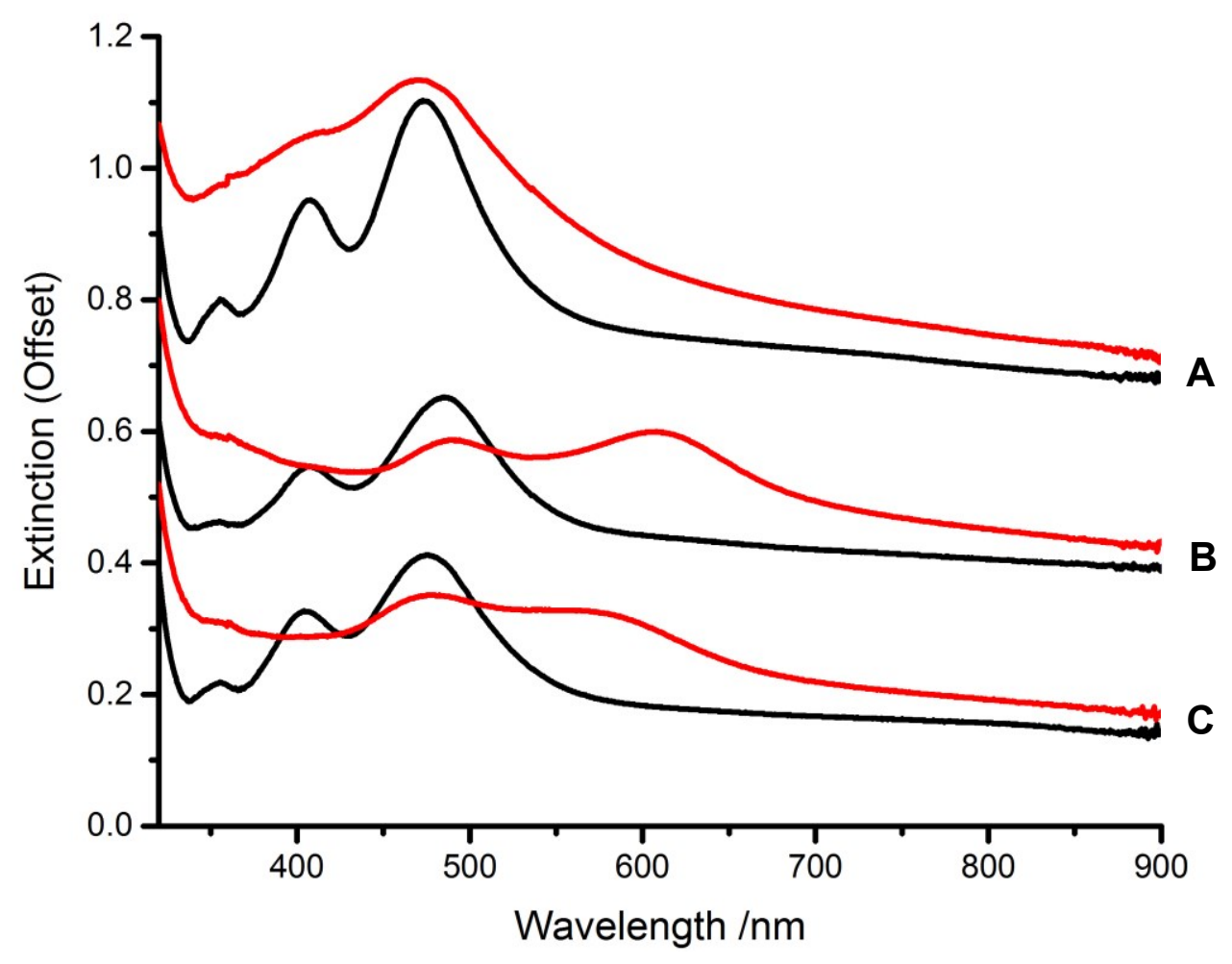

Figure 3.33: UV-Vis spectra of embedded silver nanocube monolayers before (Black) and after copper oxide growth (Red) for different added amounts of $5 \mathrm{M} \mathrm{NaOH}, 4 \mathrm{M}$ lactic acid and $0.5 \mathrm{M}$ $\mathrm{Cu}\left(\mathrm{NO}_{3}\right)_{2}$ (A) $550 \mu \mathrm{L}$ (slide 23_3), (B) $450 \mu \mathrm{L}$ (slide 5_4), (C) $350 \mu \mathrm{L}$ (slide 23_1). 


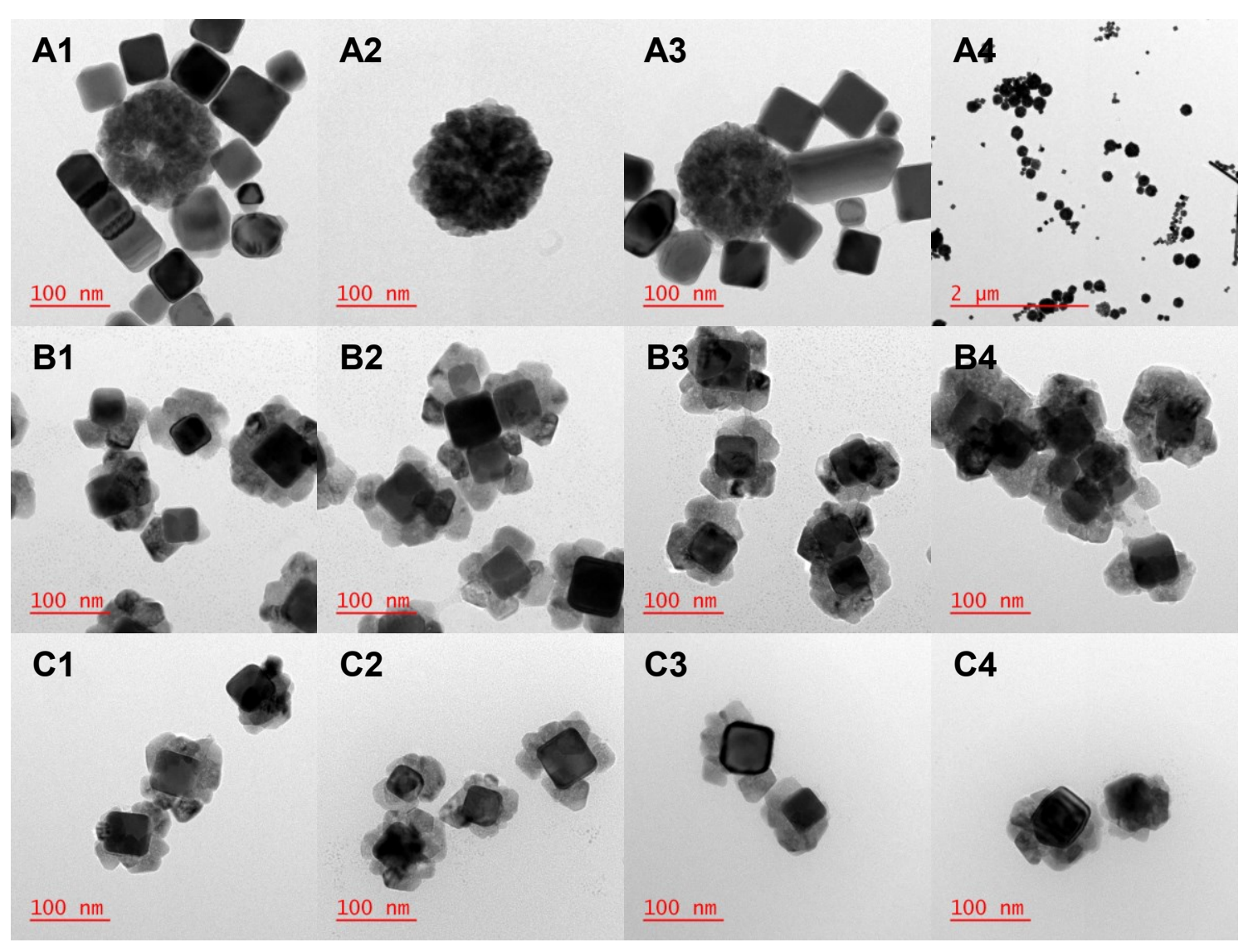

Figure 3.34: TEM images for structures produced from embedded nanocube monolayers with different added amounts of $5 \mathrm{M} \mathrm{NaOH}, 4 \mathrm{M}$ lactic acid and $0.5 \mathrm{M} \mathrm{Cu}\left(\mathrm{NO}_{3}\right)_{2}$ (A) $550 \mu \mathrm{L}$ (slide 23_3), (B) $450 \mu \mathrm{L}$ (slide 5_4), (C) $350 \mu \mathrm{L}$ (slide 23_1).

\subsection{4 - Concluding Statements on the New Set-up}

With the use of the 'New Set-up', the amounts of precursors added all at once was varied. This was done with and without lactic acid. Without lactic acid, it was observed that for increasing amounts of $\mathrm{NaOH}$ and $\mathrm{Cu}\left(\mathrm{NO}_{3}\right)_{2}$, shell coverage did not increase. Contrarily, for the samples to which increasing amounts of $\mathrm{NaOH}$, lactic acid and $\mathrm{Cu}\left(\mathrm{NO}_{3}\right)_{2}$ were added, between the low and intermediate amounts the shell thickness and coverage increased; however, with the highest amounts, very little growth occurred on the particles, as nucleation had occurred in solution, resulting in many large copper oxide particles. 
This set-up was created to improve upon the initial set-ups, and work on the production of core-partial shell nanoparticles with this set-up should continue. Addition order, rate of addition and concentrations of precursors should be varied to optimize the growth of these core-partial shell nanoparticles with this set-up.

\section{4 - Conclusions and Future Directions}

In conclusion, the method used in this work, namely the use of silver nanocube monolayers supported and embedded as a template for the production of core-partial shell silver nanocube-copper oxide structures has proved successful. Throughout this work, many lessons were learned about the parameters necessary for the optimal set-up for the creation of these types of structures, as four distinct set-ups were used to produce such particles. Particularly, the set-up should exclude oxygen, have an adjustable stirring rate, be able to stir at high rates, and be easy to clean.

In addition, with the first three set-ups, the results of slowly adding either hydrazine or the copper precursor, with and without lactic acid, was investigated. Some trends are observed for the order and rate of addition of reagents across set-ups; however of these three set-ups the structures produced with the Puck are the most similar between the different trials. Compared to the Mechanical Stirrer and Teflon Piece, the Puck spins at a much slower rate.

Generally, in the syntheses with lactic acid, where hydrazine was added slowly, the produced shells tended to be more porous, and thicker than those produced where the copper precursor was added slowly. Thinner shells are attributed to hyrdrazine reacting with oxygen and oxygen reforming $\mathrm{Cu}^{2+},{ }^{119}$ inhibiting the shell growth. In the absence of 
lactic acid, the produced copper oxide structures generally were cubic, or semi-cubic for both the slow addition or hydrazine and the slow addition of copper nitrate. This could be the result of producing the lowest energy facet, the $\{100\}$ facet, for copper oxide..$^{70}$

From the results, the New Set-up was created, and it was attempted to control the sizes of the produced shells by varying the concentrations of precursors added to the solution, with and without lactic acid. Without lactic acid, at all concentrations of precursors, very little growth was observed on the particles. With lactic acid, at the two lower concentrations the produced shells were made of many particles which had come together to make up the shell. However, for the highest concentration with lactic acid, essentially no growth was observed on the surface; however, large copper oxide nanoparticles nucleated and grew in-solution.

The core-partial shell nanoparticles produced in this work were characterized with UV-Vis spectroscopy, and it was observed that hybridization of the plasmonic modes occurred when the particles were suspended in solution, due to the partial copper oxide shell. This result is consistent with FDTD calculations using similar structures, a $70 \mathrm{~nm}$ silver nanocube with a triangular shell grown at different depths. In particular, it is observed in the calculated spectra that the hybridization is greatest for the lowest shell coverage. In this work the hybridization of the plasmonic modes expected from FDTD calculations was observed.

Further research on the production of these types of particles must be continued with the New Set-up. In particular, a similar investigation to the one carried out with the initial three set-ups must be conducted, to determine the effects that modifying the order of addition of reagents and speed of addition has on the morphologies of the produced 
structures, under conditions where there will not be interference on shell growth from oxygen. Subsequently, effort should be made to investigate the effects of other capping and structural guiding agents, which should allow controllable, shaped shell structures to be produced. Finally, the concentrations of the precursor should be varied, to determine the effects on the produced shell sizes and morphologies. The synthesis of these types of particles should then be scaled-up and to allow their use in applications, including catalysis and directed assembly.

These particles can be used for applications in sensing. In-solution hybridization of the plasmonic modes resulted in hybrid D and hybrid Q modes, which are localized towards and away from the greater refractive index dielectric, respectively, and so are sensitive to different areas. This allows for sensing specific to different areas of the particles, which can provide information about refractive index changes near the surface of the particle, monitored by the shift in the hybrid D and Q modes.

The core-partial shell nanoparticles were prepared using supported and embedded silver nanocubes. The particles produced with greater shell coverage, such as those obtained by growing the shells on supported nanoparticles with the Mechanical stirrer using lactic acid, have potential for directed assembly. Since, in this morphology only one face is exposed, this face can be functionalized to organize two particles into dimers, or to attach the particles to a surface. With the structures prepared from partially embedded nanoparticles, it is possible that attachment could occur on the exposed sides of the nanoparticle and would then be less controllable. However, particles which yield better hybridization, which is expected for less shell coverage, have greater potential applications in sensing. 
The shells grown in this work were produced with a variety of morphologies, each of which has the potential for use in different applications. Porous shell and rough shell structures possess a greater surface area, and so could potentially be useful for catalysis by the copper oxide shell. As well, the facets do not have the same surface termination, and so each is more suited for certain applications. For instance, the $\{111\}$ facet is superior to the $\{100\}$ facet in terms of antibacterial applications for E. coli bacteria. ${ }^{72}$ The $\{110\}$ facet exhibits higher photocatalytic activity for the degradation of dye pollutant methyl orange than either the $\{111\}$ or $\{100\}$ facet. ${ }^{35}$ In addition, many compounds have different affinity for certain facets, which has been employed for selective etching or deposition of a material onto the facets which have lower surface coverage, resulting in fine control over the produced structures. ${ }^{70}$ Hence, the core-partial shell nanoparticles produced have the potential for use in a variety of applications.

In addition, other materials should be investigated for the production of these types of core-shell particles, which has already started, since currently underway in the group is the investigation of core-partial shell nanoparticles with other shell materials including gold and silver sulfide. This research should be further expanded to the use of other metal oxides as the shell, including silica and can be expanded to the use of other core materials and differently shaped particles. Subsequently, these core-partial shell particles can be used to make other particles with unique shapes, for example, after removal, another material could be grown on the surface, producing a core-Janus shell particle. Contrarily, after removal, etching the core particles could produce 'nanocup' particles of the shell material. 


\section{5 - References}

(1) Burrows, N. D.; Vartanian, A. M.; Abadeer, N. S.; Grzincic, E. M.; Jacob, L. M.; Lin, W.; Li, J.;

Dennison, J. M.; Hinman, J. G.; Murphy, C. J. Anisotropic Nanoparticles and Anisotropic Surface

Chemistry. J. Phys. Chem. Lett. 2016, 7, 632-641.

(2) Murphy, C. J.; Sau, T. K.; Gole, A. M.; Orendoff, C. J.; Gao, J.; Gou, L.; Hunyadi, S. E.; Li, T. Anisotropic Metal Nanoparticles: Synthesis, Assembly and Optical Applications. J. Phys. Chem B. 2005, 109, 13857-13870.

(3) Olenin, a. Y.; Krutyakov, Y. a.; Lisichkin, G. V. Formation Mechanisms of Anisotropic Silver Nanostructures in Polyol Synthesis. Nanotechnologies Russ. 2010, 5, 421-426.

(4) Scarabelli, L.; Coronado-Puchau, M.; Giner-Casares, J. J.; Langer, J.; Liz-Marz??n, L. M. Monodisperse Gold Nanotriangles: Size Control, Large-Scale Self-Assembly, and Performance in Surface-Enhanced Raman Scattering. ACS Nano 2014, 8, 5833-5842.

(5) Kirkeminde, A.; Ruzicka, B. a; Wang, R.; Puna, S.; Zhao, H.; Ren, S. Synthesis and Optoelectronic Properties of Two-Dimensional FeS2 Nanoplates. ACS Appl. Mater. Interfaces 2012, 4, 11741177.

(6) Wiley, B. J.; Chen, Y.; McLellan, J. M.; Xiong, Y.; Li, Z. Y.; Ginger, D.; Xia, Y. Synthesis and Optical Properties of Silver Nanobars and Nanorice. Nano Lett. 2007, 7, 1032-1036.

(7) Wei, H.; Reyes-Coronado, A.; Nordlander, P.; Aizpurua, J.; Xu, H. Multipolar Plasmon Resonances in Individual Ag Nanorice. ACS Nano 2010, 4, 2649-2654.

Zhang, Q.; Large, N.; Wang, H. Gold Nanoparticles with Tipped Surface Structures as Substrates for Single-Particle Surface-Enhanced Raman Spectroscopy: Concave Nanocubes, Nanotrisoctahedra, and Nanostars. ACS Appl. Mater. Interfaces 2014, 6, 17255-17267.

(9) Barbosa, S.; Agrawal, A.; Rodríguez-Lorenzo, L.; Pastoriza-Santos, I.; Alvarez-Puebla, R. A.; Kornowski, A.; Weller, H.; Liz-Marzán, L. M. Tuning Size and Sensing Properties in Colloidal Gold Nanostars. Langmuir 2010, 26, 14943-14950. 
(10) McConnell, M. D.; Kraeutler, M. J.; Yang, S.; Composto, R. J. Patchy and Multiregion Janus Particles with Tunable Optical Properties. Nano Lett. 2010, 10, 603-609.

(11) Gilroy, K. D.; Ruditskiy, A.; Peng, H.-C.; Qin, D.; Xia, Y. Bimetallic Nanocrystals: Syntheses, Properties, and Applications. Chem. Rev. 2016.

Pothorszky, S.; Zámbó, D.; Deák, A. Structural and Optical Properties of Gold/Silica "Mushroom” Particles Prepared by Interfacial Templating. Part. Part. Syst. Charact. 2016, 1600291.

(13) Passas-Lagos, E.; Schüth, F. Amphiphilic Pickering Emulsifiers Based on Mushroom-Type Janus Particles. Langmuir 2015, 7749-7757.

(14) Ruhland, T. M.; Gröschel, A. H.; Walther, A.; Müller, A. H. E. Janus Cylinders at Liquid-Liquid Interfaces. Langmuir 2011, 27, 9807-9814.

(15) Wang, Y.; Deprince, A. E.; K. Gray, S.; Lin, X. M.; Pelton, M. Solvent-Mediated End-to-End Assembly of Gold Nanorods. J. Phys. Chem. Lett. 2010, 1, 2692-2698.

(16) Onishi, S.; Tokuda, M.; Suzuki, T.; Minami, H. Preparation of Janus Particles with Different Stabilizers and Formation of One-Dimensional Particle Arrays. Langmuir 2015, 31, 674-678.

(17) Ren, B.; Ruditskiy, A.; Song, J. H.; Kretzschmar, I. Assembly Behavior of Iron Oxide-Capped Janus Particles in a Magnetic Field. Langmuir 2012, 28, 1149-1156.

(18) Fu, X.; Wang, Y.; Huang, L.; Sha, Y.; Gui, L.; Lai, L.; Tang, Y. Assemblies of Metal Nanoparticles and Self-Assembled Peptide Fibrils_-Formation of Double Helical and Single-Chain Arrays of Metal Nanoparticles. Adv. Mater. 2003, 15, 902-906.

(19) Stewart, A. F.; Lee, A.; Ahmed, A.; Ip, S.; Kumacheva, E.; Walker, G. C. Rational Design for the Controlled Aggregation of Gold Nanorods via Phospholipid Encapsulation for Enhanced Raman Scattering. ACS Nano 2014, 8, 5462-5467.

Bharti, B.; Rutkowski, D.; Han, K.; Kumar, A. U.; Hall, C. K.; Velev, O. D. Capillary Bridging as a Tool for Assembling Discrete Clusters of Patchy Particles. J. Am. Chem. Soc. 2016, 138, 1494814953.

(21) Stewart, M. E.; Anderton, C. R.; Thompson, L. B.; Maria, J.; Gray, S. K.; Rogers, J. A.; Nuzzo, R. 
G. Nanostructured Plasmonic Sensors. Chem. Rev. 2008, 108, 494-521.

(22) Vogele, K.; List, J.; Pardatscher, G.; Holland, N. B.; Simmel, F. C.; Pirzer, T. A Self-Assembled Active Plasmonic Waveguide with a Peptide-Based Thermo-Mechanical Switch. ACS Nano 2016, acsnano.6b06635.

Lal, S.; Link, S.; Halas, N. J. Nano-Optics from Sensing to Waveguiding. Nat. Photonics 2007, 1, $641-648$.

(24) Burrows, N. D.; Lin, W.; Hinman, J. G.; Dennison, J. M.; Vartanian, A. M.; Abadeer, N. S.; Grzincic, E. M.; Jacob, L. M.; Li, J.; Murphy, C. J. Surface Chemistry of Gold Nanorods. Langmuir 2016, 32, 9905-9921.

Nie, Z.; Fava, D.; Kumacheva, E.; Zou, S.; Walker, G. C.; Rubinstein, M. Self-Assembly of MetalPolymer Analogues of Amphiphilic Triblock Copolymers. Nat. Mater. 2007, 6, 609-614.

(26) Caswell, K. K.; Wilson, J. N.; Bunz, U. H. F.; Murphy, C. J. Preferential End-to-End Assembly of Gold Nanorods by Biotin- Streptavidin Connectors. J. Am. Chem. Soc. 2003, 125, 13914-13915.

(27) Klinkova, A.; Thérien-Aubin, H.; Ahmed, A.; Nykypanchuk, D.; Choueiri, R. M.; Gagnon, B.; Muntyanu, A.; Gang, O.; Walker, G. C.; Kumacheva, E. Structural and Optical Properties of SelfAssembled Chains of Plasmonic Nanocubes. Nano Lett. 2014, 14, 6314-6321.

(28) Klinkova, A.; Thérien-Aubin, H.; Choueiri, R. M.; Rubinstein, M.; Kumacheva, E. Colloidal Analogs of Molecular Chain Stoppers. Proc. Natl. Acad. Sci. 2013, 110, 18775-18779.

(29) Noguchi, T. G.; Iwashita, Y.; Kimura, Y. Dependence of the Internal Structure on Water/Particle Volume Ratio in an Amphiphilic Janus Particle - Water - Oil Ternary System: From Micelle-like Clusters to Emulsions of Spherical Droplets. Langmuir 2017, 1030-1036.

(30) Fujii, S.; Yokoyama, Y.; Miyanari, Y.; Shiono, T.; Ito, M.; Yusa, S. I.; Nakamura, Y. MicrometerSized Gold-Silica Janus Particles as Particulate Emulsifiers. Langmuir 2013, 29, 5457-5465.

(31) Glaser, N.; Adams, D. J.; Böker, A.; Krausch, G. Janus Particles at Liquid-Liquid Interfaces. Langmuir 2006, 22, 5227-5229.

(32) Kaewsaneha, C.; Tangboriboonrat, P.; Polpanich, D.; Eissa, M.; Elaissari, A. Janus Colloidal 
Particles: Preparation, Properties, and Biomedical Applications. ACS Appl. Mater. Interfaces 2013, $5,1857-1869$.

Meng, X.; Liu, L.; Ouyang, S.; Xu, H.; Wang, D.; Zhao, N.; Ye, J. Nanometals for Solar-to-

Chemical Energy Conversion: From Semiconductor-Based Photocatalysis to Plasmon-Mediated Photocatalysis and Photo-Thermocatalysis. Adv. Mater. 2016, 1-23.

(34) Zhang, Q.; Lee, I.; Joo, J. I. B.; Zaera, F. Core-Shell Nanostructured Catalysts. Acc. Chem. Res. 2013, 46 .

Huang, W. C.; Lyu, L. M.; Yang, Y. C.; Huang, M. H. Synthesis of Cu2O Nanocrystals from Cubic to Rhombic Dodecahedral Structures and Their Comparative Photocatalytic Activity. J. Am. Chem. Soc. 2012, 134, 1261-1267.

Hara, M.; Kondo, T.; Komoda, M.; Ikeda, S.; Shinohara, K.; Tanaka, A.; Kondo, J. N.; Domen, K.; Hara, M.; Shinohara, K.; et al. Cu $2 \mathrm{O}$ as a Photocatalyst for Overall Water Splitting under Visible Light Irradiation. Chem. Commun. 1998, 2, 357-358.

Willets, K. a; Van Duyne, R. P. Localized Surface Plasmon Resonance Spectroscopy and Sensing. Annu. Rev. Phys. Chem. 2007, 58, 267-297.

Lohse, S. E.; Murphy, C. J. The Quest for Shape Control: A History of Gold Nanorod Synthesis.

Chem. Mater. 2013, 25, 1250-1261.

Rycenga, M.; Cobley, C. M.; Zeng, J.; Li, W.; Moran, C. H.; Zhang, Q.; Qin, D.; Xia, Y. Controlling the Synthesis and Assembly of Silver Nanostructures for Plasmonic Applications. Chem. Rev. 2011, 111,3669-3712.

Sajanlal, P. R.; Sreeprasad, T. S.; Samal, A. K.; Pradeep, T. Anisotropic Nanomaterials: Structure, Growth, Assembly, and Functions. Nano Rev. 2011, 5883.

(41) Sun, Y.; Mayers, B.; Herricks, T.; Xia, Y. Polyol Synthesis of Uniform Silver Nanowires: A Plausible Growth Mechanism and the Supporting Evidence. Nano Lett. 2003, 3, 955-960.

(42) Wiley, B. J.; Xiong, Y.; Li, Z.-Y.; Yin, Y.; Xia, Y. Right Bipyramids of Silver: A New Shape Derived from Single Twinned Seeds. Nano Lett. 2006, 6, 765-768. 
(43) Xia, X.; Zeng, J.; Oetjen, L. K.; Li, Q.; Xia, Y. Quantitative Analysis of the Role Played by Poly(vinylpyrrolidone) in Seed-Mediated Growth of Ag Nanocrystals. J. Am. Chem. Soc. 2012, 134, 1793-1801.

(44) Xiong, Y.; Washio, I.; Chen, J.; Cai, H.; Li, Z.; Xia, Y. Poly (Vinyl Pyrrolidone): A Dual

Functional Reductant and Stabilizer for the Facile Synthesis of Noble Metal Nanoplates in Aqueous Solutions. Langmuir 2006, 8563-8570.

Cortie, M. B.; McDonagh, A. M. Synthesis and Optical Properties of Hybrid and Alloy Plasmonic Nanoparticles. Chem. Rev. 2011, 111, 3713-3735.

Yang, Y.; Liu, J.; Fu, Z.-W.; Qin, D. Galvanic Replacement-Free Deposition of Au on Ag for CoreShell Nanocubes with Enhanced Chemical Stability and SERS Activity. J. Am. Chem. Soc. 2014, $136,8153-8156$.

Niitsoo, O.; Couzis, A. Facile Synthesis of Silver Core - Silica Shell Composite Nanoparticles. J. Colloid Interface Sci. 2011, 354, 887-890.

Jing, H.; Large, N.; Zhang, Q.; Wang, H. Epitaxial Growth of Cu2O on Ag Allows for Fine Control over Particle Geometries and Optical Properties of Ag-Cu2O Core-Shell Nanoparticles. J. Phys. Chem. C 2014.

Khodashenas, B.; Ghorbani, H. R. Synthesis of Silver Nanoparticles with Different Shapes. Arab. J. Chem. 2015.

Obare, S. O.; Jana, N. R.; Murphy, C. J. Preparation of Polystyrene- and Silica-Coated Gold Nanorods and Their Use as Templates for the Synthesis of Hollow Nanotubes. Nano Lett. 2001, 1, $601-603$.

Jiang, R.; Qin, F.; Liu, Y.; Ling, X. Y.; Guo, J.; Tang, M.; Cheng, S.; Wang, J. Colloidal Gold Nanocups with Orientation-Dependent Plasmonic Properties. Adv. Mater. 2016, 6322-6331.

Turkevich, J.; Stevenson, P. C.; Hillier, J. A Study of the Nucleation and Growth Processes in the Synthesis of Colloidal Gold. Discuss. Faraday Soc 1951, 11. 
J. Mater. Sci. Appl. 2015, 4, 325.

(54) Xia, Y.; Xiong, Y.; Lim, B.; Skarabalak, S. E. Shape-Controlled Synthesis of Metal Nanocrystals : Simple Chemistry Meets Complex Physics? 2009, 48, 60-103.

(55) Chang, S.; Chen, K.; Hua, Q.; Ma, Y.; Huang, W. Evidence for the Growth Mechanisms of Silver Nanocubes and Nanowires. J. Phys. Chem. C 2011, 115, 7979-7986.

(56) Siekkinen, A. R.; McLellan, J. M.; Chen, J.; Xia, Y. Rapid Synthesis of Small Silver Nanocubes by Mediating Polyol Reduction with a Trace Amount of Sodium Sulfide or Sodium Hydrosulfide. Chem. Phys. Lett. 2006, 432, 491-496.

Wang, Y.; Zheng, Y.; Huang, C. Z.; Xia, Y. Synthesis of Ag Nanocubes $18-32$ Nm in Edge Length: The E Ff Ects of Polyol on Reduction Kinetics, Size Control, and Reproducibility. J. Am. Chem. Soc. 2013, 135, 1941-1951.

(58) Seo, D.; Park, J. C.; Song, H. Polyhedral Gold Nanocrystals with O H Symmetry : From Octahedra to Cubes. J. Am. Chem. Soc. 2016, 14863-14870.

Zeng, J.; Zheng, Y.; Rycenga, M.; Tao, J.; Li, Z. Y.; Zhang, Q.; Zhu, Y.; Xia, Y. Controlling the Shapes of Silver Nanocrystals with Different Capping Agents. J. Am. Chem. Soc. 2010, 132, 85528553.

(60) Koczkur, K. M.; Mourdikoudis, S.; Polavarapu, L.; Skrabalak, S. E. Polyvinylpyrrolidone (PVP) in Nanoparticle Synthesis. Dalton Trans. 2015, 44, 17883-17905.

(61) Long, R.; Zhou, S.; Wiley, B. J.; Xiong, Y. Oxidative Etching for Controlled Synthesis of Metal Nanocrystals: Atomic Addition and Subtraction. Chem. Soc. Rev. 2014, 43, 6288-6310.

(62) Zheng, Y.; Zeng, J.; Ruditskiy, A.; Liu, M.; Xia, Y. Oxidative Etching and Its Role in Manipulating the Nucleation and Growth of Noble-Metal Nanocrystals. Chem. Mater. 2014, 26, 22-33.

(63) Skrabalak, S. E.; Wiley, B. J.; Kim, M.; Formo, E. V.; Xia, Y. On the Polyol Synthesis of Silver Nanostructures: Glycolaldehyde as a Reducing Agent. Nano Lett. 2008, 8, 2077-2081.

(64) Thanh, N. T. K.; Maclean, N.; Mahiddine, S. Mechanisms of Nucleation and Growth of Nanoparticles in Solution. Chem. Rev. 2014, 114, 7610-7630. 
(65) Sun, Y. Controlled Synthesis of Colloidal Silver Nanoparticles in Organic Solutions: Empirical Rules for Nucleation Engineering. Chem. Soc. Rev. 2013, 42, 2497-2511.

(66) Xia, Y.; Xia, X.; Peng, H.-C. Shape-Controlled Synthesis of Colloidal Metal Nanocrystals:

Thermodynamic versus Kinetic Products. J. Am. Chem. Soc. 2015, 137, 7947-7966.

(67) Wiley, B.; Sun, Y.; Mayers, B.; Xia, Y. Shape-Controlled Synthesis of Metal Nanostructures: The Case of Silver. Chemistry 2005, 11, 454-463.

(68) Lignier, P.; Bellabarba, R.; Tooze, R. P. Scalable Strategies for the Synthesis of Well-Defined Copper Metal and Oxide Nanocrystals. Chem. Soc. Rev. 2012, 41, 1708-1720.

(69) Wang, Z.; Wang, H.; Wang, L.; Pan, L. Controlled Synthesis of Cu 2 O Cubic and Octahedral Nano- and Microcrystals. Cryst. Res. Technol. 2009, 44, 624-628.

(70) Shang, Y.; Guo, L. Facet-Controlled Synthetic Strategy of Cu2O-Based Crystals for Catalysis and Sensing. Adv. Sci. 2015, 2, 1500140.

(71) Majhi, S. M.; Rai, P.; Raj, S.; Chon, B.-S.; Park, K.-K.; Yu, Y.-T. Effect of Au Nanorods on Potential Barrier Modulation in Morphologically Controlled Au@Cu2O Core-Shell Nanoreactors for Gas Sensor Applications. Appl. Mater. Interfaces 2014, 6, 7491-7497.

(72) Ren, J.; Wang, W.; Sun, S.; Zhang, L.; Wang, L.; Chang, J. Crystallography Facet-Dependent Antibacterial Activity: The Case of Cu2O. Ind. Eng. Chem. Res. 2011, 10366-10369.

(73) Susman, M. D.; Feldman, Y.; Vaskevich, A.; Rubinstein, I. Chemical Deposition of Cu2O Nanocrystals with Precise Morphology Control. ACS Nano 2014, 8, 162-174.

(74) Wu, W.; Feng, K.; Shan, B.; Zhang, N. Orientation and Grain Shape Control of Cu2O Film and the Related Properties. Electrochim. Acta 2015, 176, 59-64.

(75) Zhang, D. F.; Zhang, H.; Guo, L.; Zheng, K.; Han, X. D.; Zhang, Z. Delicate Control of Crystallographic Facet-Oriented Cu2O Nanocrystals and the Correlated Adsorption Ability. $J$. Mater. Chem. 2009, 19, 5220-5225.

(76) Xu, H.; Wang, W.; Zhu, W. Shape Evolution and Size-Controllable Synthesis of Cu2O Octahedra and Their Morphology-Dependent Photocatalytic Properties. J. Phys. Chem. B 2006, 110, 13829- 
13834.

(77) Ke, W.-H.; Hsia, C.-F.; Chen, Y.-J.; Huang, M. H. Synthesis of Ultrasmall Cu2 O Nanocubes and Octahedra with Tunable Sizes for Facet-Dependent Optical Property Examination. Small 2016, 12, $3530-3534$.

(78) Jimenez-Cadena, G.; Comini, E.; Ferroni, M.; Sberveglieri, G. Synthesis of Cu2O Bi-Pyramids by Reduction of $\mathrm{Cu}(\mathrm{OH}) 2$ in Solution. Mater. Lett. 2010, 64, 469-471.

(79) Yang, Z.; Sun, S.; Kong, C.; Song, X.; Ding, B. Designated-Tailoring on $\{100\}$ Facets of Cu2O Nanostructures: From Octahedral to Its Different Truncated Forms. J. Nanomater. 2010, 2010, 111.

(80) Gou, L.; Murphy, C. J. Solution-Phase Synthesis of Cu2O Nanocubes. Nano Lett. 2003, 3, 231234.

(81) Zhao, H. Y.; Wang, Y. F.; Zeng, J. H. Hydrothermal Synthesis of Uniform Cuprous Oxide Microcrystals with Controlled Morphology. Cryst. Growth Des. 2008, 8, 3731-3734.

(82) Kuo, C.; Huang, M. H.; Kuo, C.; Huang, M. H. Facile Synthesis of Cu2O Nanocrystals with Systematic Shape Evolution from Cubic to. J. Phys. Chem. C 2008, 18355-18360.

Zhao, X.; Bao, Z.; Sun, C.; Xue, D. Polymorphology Formation of Cu2O: A Microscopic Understanding of Single Crystal Growth from Both Thermodynamic and Kinetic Models. J. Cryst. Growth 2009, 311, 711-715.

(84) Hwee, C.; Ng, B.; Fan, W. Y. Shape Evolution of Cu 2 O Nanostructures via Kinetic and Thermodynamic Controlled Growth. J. Phys. Chem. B 2006, 110, 20801-20807.

Sui, Y.; Fu, W.; Yang, H.; Zeng, Y.; Zhang, Y.; Zhao, Q.; Li, Y.; Zhou, X.; Leng, Y.; Li, M.; et al. Low Temperature Synthesis of Cu2O Crystals: Shape Evolution and Growth Mechanism. Cryst. Growth Des. 2010, 10, 99-108.

(86) Ho, J. Y.; Huang, M. H. Synthesis of Submicrometer-Sized Cu2O Crystals with Morphological Evolution from Cubic to Hexapod Structures and Their Comparative Photocatalytic Activity. $J$. Phys. Chem. C 2009, 113, 14159-14164. 
(87) Gou, L.; Murphy, C. J. Controlling the Size of Cu2O Nanocubes from 200 to 25 Nm. J. Mater. Chem. 2004, 14, 735 .

Bijani, S.; Schrebler, R.; Dalchiele, E. A.; Gabás, M.; Martínez, L.; Ramos-Barrado, J. R. Study of the Nucleation and Growth Mechanisms in the Electrodeposition of Micro- and Nanostructured Cu2O Thin Films. J. Phys. Chem. C 2011, 115, 21373-21382.

(89) Golden, T. D.; Shumsky, M. G.; Zhou, Y.; VanderWerf, R. a.; Van Leeuwen, R. a.; Switzer, J. a. Electrochemical Deposition of Copper(I) Oxide Films. Chem. Mater. 1996, 8, 2499-2504.

(90) Jing, H.; Large, N.; Zhang, Q.; Wang, H. Epitaxial Growth of Cu $2 \mathrm{O}$ on Ag Allows for Fine Control Over Particle Geometries and Optical Properties of Ag-Cu 2 O Core-Shell Nanoparticles. J. Phys. Chem. C 2014, 118, 19948-19963.

(91) Tsuji, M.; Yamaguchi, D.; Matsunaga, M.; Alam, M. J. Epitaxial Growth of Au@Cu Core-Shell Nanocrystals Prepared Using the PVP-Assisted Polyol Reduction Method. Cryst. Growth Des. 2010, $10,5129-5135$.

(92) Tsuji, M.; Yamaguchi, D.; Matsunaga, M.; Ikedo, K. Epitaxial Growth of Au@Ni Core-Shell Nanocrystals Prepared Using a Two-Step Reduction Method. Cryst. Growth Des. 2011, 11, 19952005.

(93) Lal, S.; Link, S.; Halas, N. J. Nano-Optics from Sensing to Waveguiding. Nat. Photonics 2007, 1, $641-648$.

(94) Lee, Y. H.; Chen, H.; Xu, Q.-H.; Wang, J. Refractive Index Sensitivities of Noble Metal Nanocrystals: The Effects of Multipolar Plasmon Resonances and the Metal Type. J. Phys. Chem. C 2011, 115, 7997-8004.

(95) Wiley, B. J.; Im, S. H.; Li, Z.-Y. Y.; McLellan, J.; Siekkinen, A.; Xia, Y. Maneuvering the Surface Plasmon Resonance of Silver Nanostructures through Shape-Controlled Synthesis. J. Phys. Chem. Chem. B 2006, 110, 15666-15675.

(96) Zhang, Q.; Li, W.; Moran, C.; Zeng, J.; Chen, J.; Re, V. Seed-Mediated Synthesis of Ag Nanocubes with Controllable Edge Lengths in the Range of 30 - $200 \mathrm{Nm}$ and Comparison of Their Optical 
Properties. 2010, 11372-11378.

(97) Zhang, S.; Bao, K.; Halas, N. J.; Xu, H.; Nordlander, P. Substrate-Induced Fano Resonances of a Plasmonic Nanocube: A Route to Increased-Sensitivity Localized Surface Plasmon Resonance Sensors Revealed. Nano Lett. 2011, 11, 1657-1663.

(98) Miller, M. M.; Lazarides, A. A. Sensitivity of Metal Nanoparticle Surface Plasmon Resonance to the Dielectric Environment. J. Opt. A Pure Appl. Opt. 2005, 109, 21556-21565.

(99) Hutter, E.; Fendler, J. H. Exploitation of Localized Surface Plasmon Resonance. Adv. Mater. 2004, $16,1685-1706$.

(100) Schlücker, S. Surface-Enhanced Raman Spectroscopy: Concepts and Chemical Applications. Angew. Chem. Int. Ed. Engl. 2014, 53, 4756-4795.

(101) Noguez, C. Surface Plasmons on Metal Nanoparticles: The Influence of Shape and Physical Environment. J. Phys. Chem. C 2007, 111, 3806-3819.

(102) Mulvaney, P. Surface Plasmon Spectroscopy of Nanosized Metal Particles. Langmuir 1996, 12, $788-800$.

(103) Kelly, K. L.; Coronado, E.; Zhao, L. L.; Schatz, G. C. The Optical Properties of Metal Nanoparticles: The Influence of Size, Shape and Dielectric Environment. 2003, 107, 668-677.

(104) Mayer, K. M.; Hafner, J. H.; Antigen, A. À. Localized Surface Plasmon Resonance Sensors. Chem. Rev. 2011, 111, 3828-3857.

(105) Jensen, T. R.; Duval, M. L.; Kelly, K. L.; Lazarides, A. A.; Schatz, G. C.; Van Duyne, R. P. Nanosphere Lithography: Effect of the External Dielectric Medium on the Surface Plasmon Resonance Spectrum of a Periodic Array of Silver Nanoparticles. J. Phys. Chem. B 1999, 103, 9846-9853.

(106) Knight, M. W.; Wu, Y.; Lassiter, J. B.; Nordlander, P.; Halas, N. J. Substrates Matter: Influence of an Adjacent Dielectric on an Individual Plasmonic Nanoparticle. Nano Lett. 2009, 9, 2188-2192.

(107) Bottomley, A.; Prezgot, D.; Ianoul, A. Plasmonic Properties of Silver Nanocube Monolayers on High Refractive Index Substrates. Appl. Phys. A 2012, 109, 869-872. 
(108) Acharya, S.; Hill, J. P.; Ariga, K. Soft Langmuir-Blodgett Technique for Hard Nanomaterials. $A d v$. Mater. 2009, 21, 2959-2981.

(109) Martin, P.; Szablewski, M. NIMA Langmuir-Blodgett Troughs Operating Manual 6th Edition; 2004.

(110) Ahamad, N.; Ianoul, A. Using Phospholipids To Control Interparticle Distance in SERS-Active Substrates. J. Phys. Chem. C 2011, 115, 3587-3594.

(111) Rubinger, C. P. L.; Moreira, R. L.; Cury, L. A.; Fontes, G. N.; Neves, B. R. A.; Meneguzzi, A.; Ferreira, C. A. Langmuir-Blodgett and Langmuir-Schaefer Films of poly(5-Amino-1-Naphthol) Conjugated Polymer. Appl. Surf. Sci. 2006, 253, 543-548.

(112) Overney, R. M.; Buenviaje, C.; Luginbühl, R.; Dinelli, F. GLASS AND STRUCTURAL TRANSITIONS MEASURED AT POLYMER SURFACES ON THE NANOSCALE. $J$. Therm. Anal. Calorim. 2000, 59, 205-225.

(113) Tsui, O. K. C.; Russell, T. P.; Hawker, C. J. Effect of Interfacial Interactions on the Glass Transition of Polymer Thin Films. Macromolecules 2001, 34, 5535-5539.

(114) Vignaud, G.; Chebil, M. S.; Bal, J. K.; Delorme, N.; Beuvier, T.; Grohens, Y.; Gibaud, A. Densification and Depression in Glass Transition Temperature in Polystyrene Thin Films. Langmuir 2014, 11599-11608.

(115) Ellison, C. J.; Torkelson, J. M. The Distribution of Glass-Transition Temperatures in Nanoscopically Confined Glass Formers. Nat. Mater. 2003, 2, 695-700.

(116) Ediger, M. D.; Forrest, J. A. Dynamics near Free Surfaces and the Glass Transition in Thin Polymer Films: A View to the Future. Macromolecules 2014, 47, 471-478.

(117) Bottomley, A.; Prezgot, D.; Coyle, J. P.; Ianoul, A. Dynamics of Nanocubes Embedding into Polymer Films Investigated via Spatially Resolved Plasmon Modes. Nanoscale 2016, 8, 1116811176.

(118) Chaudhury, M. K. Interfacial Interaction between Low Energy Surfaces. Mater. Sci. Eng. R Reports 1996, R16, 97-159 . 
(119) Gaunt, H.; Wetton, E. A. M. The Reaction between Hydrazine and Oxygen in Water. J. Appl. Chem. 1966, 16, 171-176.

(120) Rasband, W. S. ImageJ., U. S. National Institutes of Health, Bethesda, Maryland, USA, https://imagej.nih.gov/ij/, 1997-2016. 